\title{
Application of Extended Finite Element Method (XFEM) to Simulate Hydraulic Fracture Propagation from Oriented Perforations
}

By

Jay Sepehri, B.Sc.

A Thesis

in

Petroleum Engineering

\author{
Submitted to the Graduate Faculty \\ of Texas Tech University in \\ Partial fulfillment of \\ the requirements for \\ the Degree of \\ MASTER OF SCIENCE \\ In PETROLEUM ENGINEERING
}

Approved by

Dr. Mohammed Y. Soliman

Chair of Committee

Dr. Stephan M. Morse

Dr. Habib Menouar

Dr. Waylon House

Mark Sheridan

Dean of the Graduate School

May, 2014 
Copyright 2014, Jay Sepehri 


\section{Acknowledgements}

I would like to express my sincere gratitude to my advisor Dr Mohamed Soliman for his continuous support of my Master's thesis and research, for his motivation, enthusiasm, patience and great knowledge. His guidance has helped me in the research and writing of this thesis.

Besides my advisor, I would like to thank the rest of my thesis committee: Dr. Stephan M. Morse, for his constructive comments and encouragement, and Dr. Habib Menouar and Dr. Waylon House for their insightful comments and suggestions.

Also I thank all professors, classmates and staff at Bob L. Herd Department of Petroleum Engineering at Texas Tech University for the two wonderful years of education and professional and personal development.

I would particularly like to thank my fellow group mates in Hydraulic Fracturing Research Group, Ali Jamali, Ali Rezaei and Dr Mehdi Rafiee, for the inspiring comments, stimulating discussions, for the long hours we were working together on different projects, and for all the fun we had in the last two years.

Last but not the least; I owe my deepest gratitude to my family: my parents and my wife Hannah, for supporting me emotionally and financially throughout my life and during this study and for their continuous question of "When are you going to defend your thesis?"

Thank you and God bless y'all. 


\section{Table of Contents}

Acknowledgements .................................................................................................................. ii

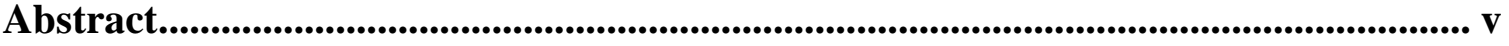

List of Tables ............................................................................................................................... vi

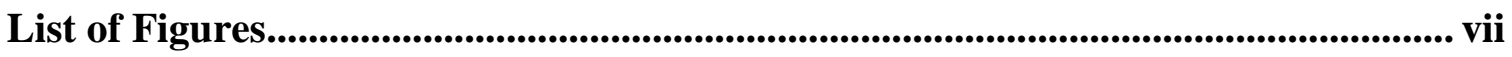

1. Introduction .................................................................................................................................. 1

2. Extended Finite Element Method............................................................................ 4

2.1 Introduction .............................................................................................. 4

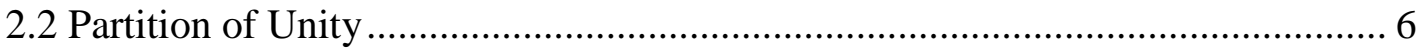

2.2.1 Partition of Unity Finite Element Method ........................................................................

2.2.2 Generalized Finite Element Method ............................................................................

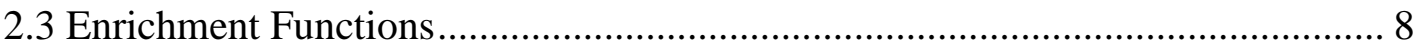

2.3.1 The Heaviside Function ...........................................................................................

2.3.2 Asymptotic Near-Tip Field Functions .............................................................................

2.4 Level Set Method for Modeling Discontinuities ........................................... 11

2.5 Numerical Integration and Convergence ................................................... 12

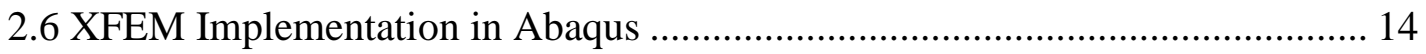

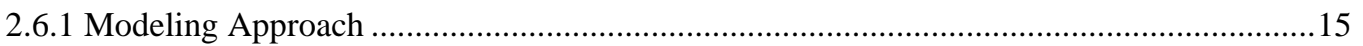

2.6.2 The Cohesive Segments Method and Phantom Nodes..........................................................15

2.6.3 The Principles of LEFM and Phantom Nodes .................................................................16

2.6.4 Virtual Crack Closure Technique................................................................................... 17

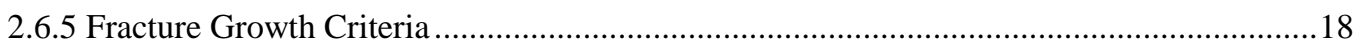

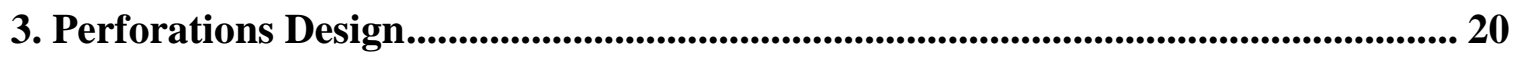

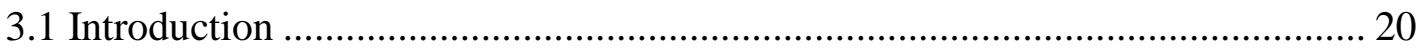

3.2 Parameters in Designing Perforation ........................................................... 20

3.2.1 Perforation Phasing .....................................................................................................22

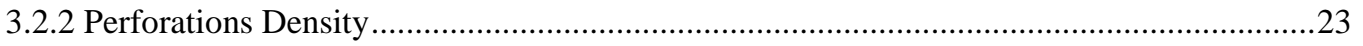

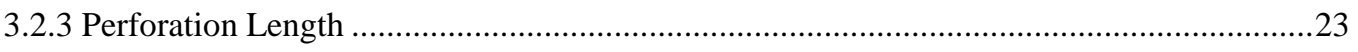

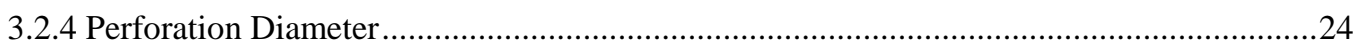




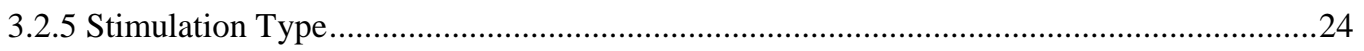

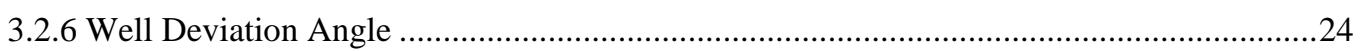

3.3 Perforating for Hydraulic Fracturing Treatment .......................................... 25

3.3.1 Minimum and Maximum Horizontal Stress Direction ..........................................................26

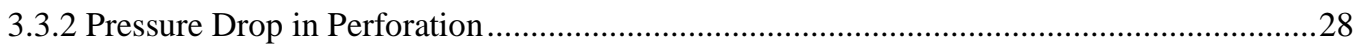

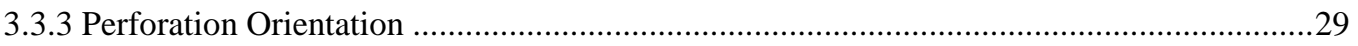

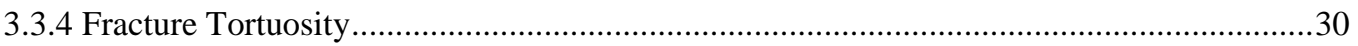

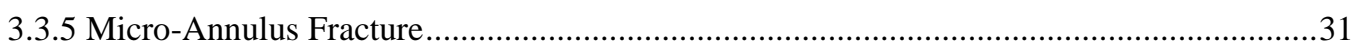

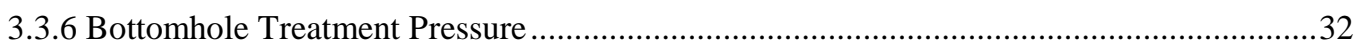

3.4 Experimental Investigation of Fracture Propagation from Perforation .............. 35

4. Modeling Fracture Propagation from Perforations ......................................................... 37

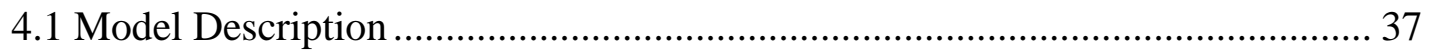

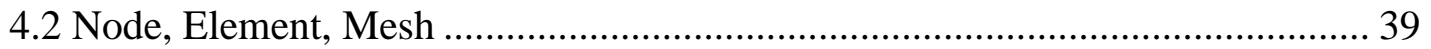

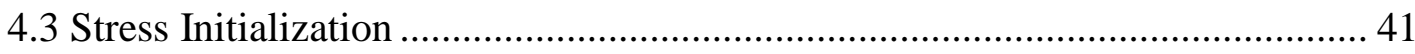

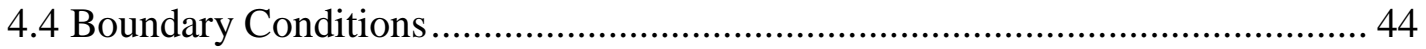

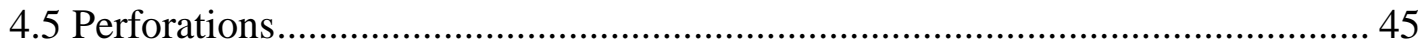

4.6 Defining Enriched Feature and its Properties in Abaqus .................................. 46

4.7 Fracture Initiation and Extension in Abaqus ............................................... 48

5. Results and Discussions ........................................................................................................... 50

5.1 Perforation Angle ................................................................................... 50

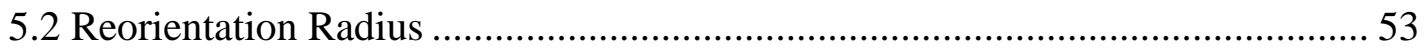

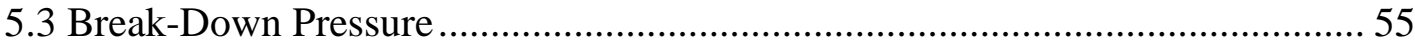

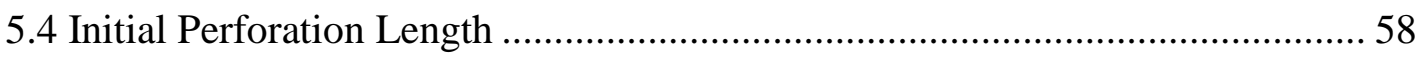

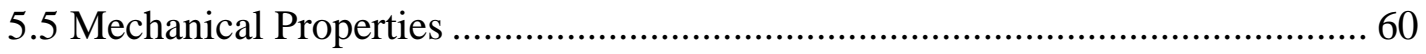

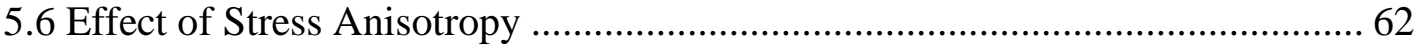

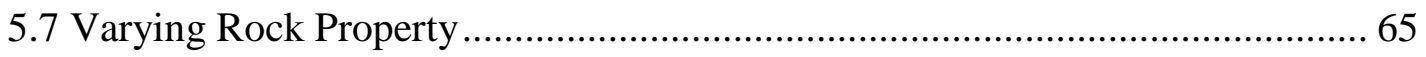

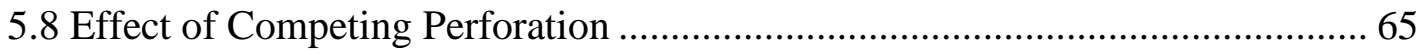

6. Conclusions and Recommendations ...................................................................... 70

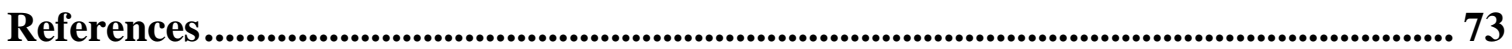




\begin{abstract}
The majority of hydraulic fracture treatments are performed in cased, perforated wells. Perforations serve as the channel of fluid communication between wellbore and formation and as a starting point for hydraulic fracture to lower breakdown pressure. Hydraulic fracture propagation is intended to be in the direction of perforations, but they are not necessarily extended in the direction initiated. If not aligned with the direction of Preferred Fracture Plane (PFP), fractures reorient to propagate parallel to the plane of the least resistance.

Extended Finite Element Method (XFEM) has been introduced as a powerful numerical tool in solving discontinuity problems to overcome the drawback of the conventional Finite Element method especially when simulating fracture propagation. Using capabilities of XFEM in commercial FE software, a model was developed to investigate the effect of perforation orientation on fracture propagation.

Different parameters and design configuration including perforation angle, perforation length, rock mechanical properties, stress anisotropy and changing medium properties are examined to better understand fracture propagation from cased perforated wells and to come up with a better perforation design when a hydraulic fracturing treatment is intended.

The results from this study showed that hydraulic fracture propagation pattern is affected by the perforation deviation from preferred fracture plane (PFP) and perforation length. As expected, horizontal stress anisotropy and rock mechanical properties were found to have a strong influence on fracture propagation from perforations. The simulation results from this study offer methods to enhance perforation design for hydraulic fracture treatment especially in the case of high stress anisotropy and high uncertainty on the preferred fracture plane.
\end{abstract}




\section{List of Tables}

4.1 Physical and mechanical properties of the sample used in the model .................... 38

4.2 Summary of some of keywords used to define enrichment in Abaqus................... 47

4.3 Damage initiation criterion and their implementation in abaqus ............................ 49

5.1 Reorientation Radius for different Perforation Angles ............................................ 54

5.2 Breakdown pressure vs perforation angle...................................................... 56 


\section{List of Figures}

2.1 Comparison of fracture path in FEM and XFEM .......................................... 5

2.2 Partition of unity of a circle with four functions............................................... 6

2.3 Evaluation of Heaviside function.................................................................. 8

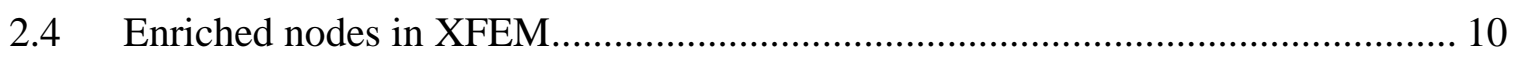

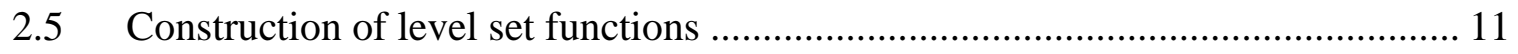

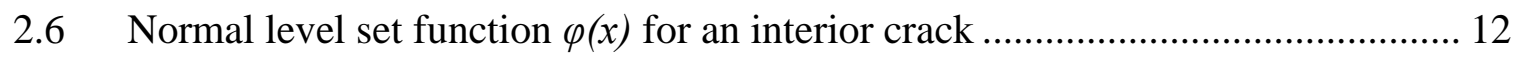

2.7 Subtriangulation of elements cut by a fracture ............................................... 13

2.8 The principle of the phantom node method .................................................... 16

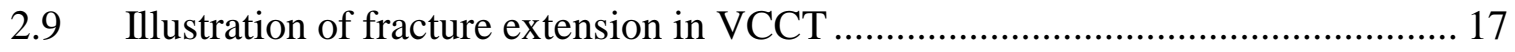

3.1 Schematic of Perforation gun and parameters ............................................... 21

3.2 Common perforation Phasing angles ............................................................ 22

3.3 Complex fracture geometry around oriented perforation ................................. 26

3.4 Ultrasonic Borehole Imager tool detects direction of wellbore breakout ............. 27

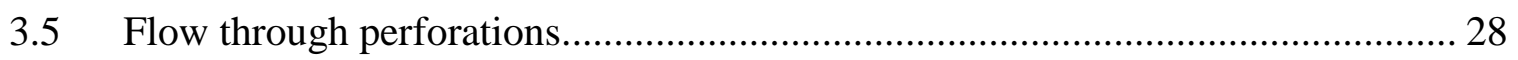

3.6 Graphical representation of wellbore, perforations and stresses ........................ 29

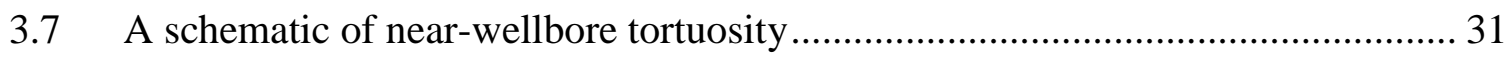

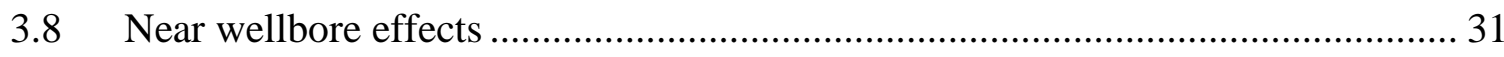

3.9 Schematic of significant pressures during hydraulic fracturing treatment ............ 33

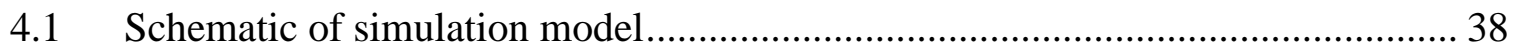

4.2 Two mesh configurations used for the analyses throughout the study ................. 40

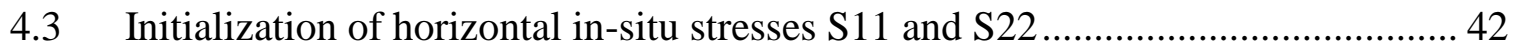

4.4 Tangential and radial stress distribution around wellbore ................................ 43

4.5 Tensor representation of principal horizontal stresses .................................... 44

4.6 Displacement boundary condition applied to the model.................................. 45

4.7 Wellbore, initial perforation and parameters involved .................................... 46

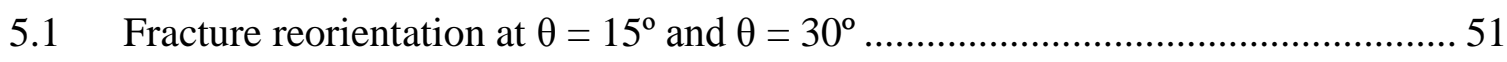

5.2 Fracture reorientation at $\theta=45^{\circ}$ and comparison to experimental model ............ 51

5.3 Fracture reorientation at $\theta=60^{\circ}$ and comparison to experimental model ............ 52 
5.4 Fracture reorientation at $\theta=75^{\circ}$ and comparison to experimental model ............52

5.5 Fracture reorientation at $\theta=90^{\circ}$ and comparison to experimental model ............ 53

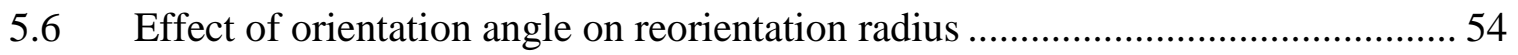

5.7 Fracture reorientation affected by stress distribution around wellbore ................ 55

5.8 Change in break down pressure with perforation angle ..................................... 56

5.9 Tangential stress around wellbore increases with increasing angle.................... 57

5.10 Tangential Stress at Perforation tip for different angles $\left(\mathrm{L}=\mathrm{r}_{\mathrm{w}}\right) \ldots \ldots \ldots \ldots \ldots \ldots \ldots . . . \ldots \ldots$

5.11 Effect of perforation length on fracture reorientation ...................................... 59

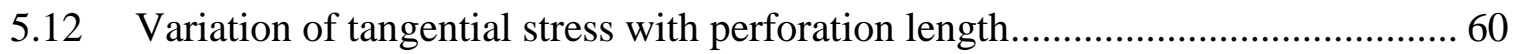

5.13 Effect of Young's Modulus on fracture propagation pattern $\left(\theta=45^{\circ}\right) \ldots \ldots \ldots \ldots \ldots . . . . . .61$

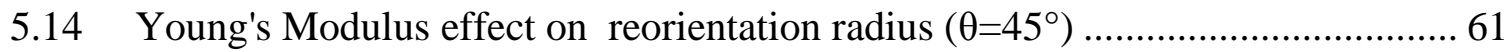

5.15 Effect of stress anisotropy on fracture propagation pattern $\left(\theta=45^{\circ}\right) \ldots \ldots \ldots \ldots \ldots . . . . . .62$

5.16 Effect of stress anisotropy on fracture propagation $\left(\theta=15^{\circ}, 30^{\circ}, 45^{\circ}\right) \ldots \ldots \ldots \ldots . . . .63$

5.17 Effect of stress anisotropy on fracture propagation $\left(\theta=60^{\circ}, 75^{\circ}, 90^{\circ}\right) \ldots \ldots \ldots \ldots .64$

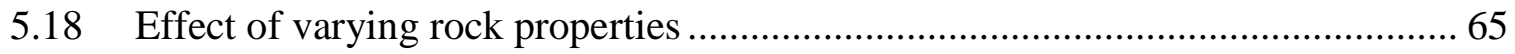

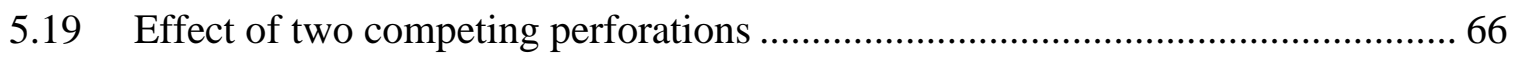

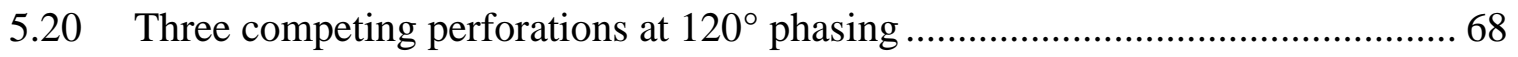

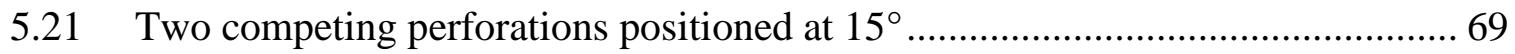

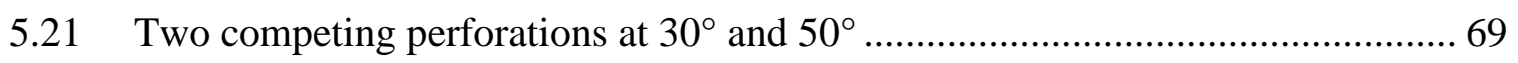


Texas Tech University, Jay Sepehri, May 2014

\section{Chapter 1}

\section{Introduction}

Production from shale formations has become one of the most rapidly expanding areas in oil and gas exploration and production industry. Because of extremely low permeability and low porosity, hydraulic fracturing treatments are necessary to maximize access to the formation and produce economic production rates. Implementation of hydraulic fracturing has changed the energy industry worldwide. Many hydrocarbon resources in low permeability shales have been developed in North America by drilling and fracturing long horizontal wells in plays such as Marcellus Shale, Barnett Shale, and also oil fields such as the Monterey formation, the Eagle Ford shale and the Wolfcamp shale oil (Oil and Shale Gas Discovery News).

Understanding fracture initiation and propagation from wellbores is essential for performing efficient hydraulic fracture stimulation treatment. Usually wellbores are cased and perforated before hydraulic fracturing treatments are performed. Perforation provides a channel of communication between the wellbore and the reservoir. In hydraulic fracture treatment, perforation can serve as an initial fracture to help with fracture initiation and controlling its propagation direction. Perforations play an important role in the complex fracture geometries around the wellbore. The initiation of a single fracture and avoiding multiple T-shaped and reoriented fractures from the wellbore is one of the main objectives of using the perforation.

Different parameters contribute to the success of hydraulic fracture treatment through perforation. Among them, perforation angle (deviation from preferred fracture plane), perforation length, stress anisotropy and nearby discontinuity play important roles and affect the hydraulic fracture propagation pattern.

Numerical simulation of the hydraulic fracturing process is an essential part of understanding the complex mechanics of hydraulic fracturing. Because of the strong nonlinearity in coupling of the fluid flow inside the fracture and fracture propagation, numerical simulation of hydraulic fracturing is a very complex problem. This problem 
gets even more complex when taking into account the existence of natural fractures, fluid leak-off to the formation and multi layers with varying physical and mechanical properties.

Great effort has been devoted to the numerical simulation of hydraulic fracturing with the first 3-D modeling efforts starting in the late 1970s. Significant progress has been made in developing 2D, pseudo-3D and 3D numerical hydraulic fracture models during the last several years.

The finite element method has been used to model hydraulic fracture propagation in heterogeneous rocks which may include nonlinear mechanical properties and may be subjected to complex boundary conditions. However, the finite element model requires remeshing after each time step for the mesh to conform exactly to the fracture geometry as the fracture propagates. This will result in higher computation time and sometimes convergence problems.

By adding special enriched shape functions and additional degrees of freedom to the standard finite element approximation, the extended finite element method (XFEM) overcomes the drawbacks associated with the use of the conventional finite element methods and enables the fracture to be represented without meshing fracture surfaces. Therefore, the fracture geometry is completely independent of the mesh configuration. As a result, remeshing is not required, which results in faster simulation of the fracture propagation. Since the introduction of this method, many new formulations and applications have appeared in the literature. The XFEM has been used to investigate hydraulic fracture problems by many authors. (Lecampion, 2009; Dahi-Taleghani and Olson, 2011; Weber et al., 2013; Chen, 2013)

This study focuses on the implementation of XFEM to simulate propagation of hydraulic fracture from a perforated cased hole. Finite Element software with XFEM capability has been implemented to investigate the effect of perforation orientations and the near wellbore stress regime on induced hydraulic fractures. This interaction will affect the orientation and geometry of fractures which is the main focus of this study.

The second chapter in this study presents a comprehensive introduction to XFEM and the fundamental concepts that make this method such a powerful tool in solving 
fracturing problems. Some concepts including partition of unity, Enrichment functions, Level Set Method and their numerical application in XFEM methods are also included.

Chapter 3 is devoted to the different aspects of cased hole perforation. After highlighting important parameters contributing to a successful perforation, considerations for hydraulic fracturing treatment are discussed.

A model to study the effect of perforation orientation is developed in chapter 4. This chapter also includes model parameters and specifications, assumptions and boundary conditions. A brief description of parameters, keywords and theory used to build this model is also included in this chapter.

In chapter 5, the results from different scenarios and configurations are presented and verified against some experimental studies available in the literature. Then different sensitivity analyses are performed to observe the effect of involving parameters.

The study is concluded in chapter 6 by conclusion drawn from the results discussed during this study and by offering recommendations for perforation design intended for hydraulic fracturing treatment as well as some research directions recommended for future work.

This study demonstrates the capabilities of extended finite element method in solving the complex problems of hydraulic fracture propagation and observing the effects of perforation orientation on induced fractures. The results from this study verified with some experimental observations in the literature gives a better understanding of the problem and contributing parameters in order to improve hydraulic fracturing treatments. This study provides a tool to investigate different parameters in designing hydraulic fracture treatment from perforated wells. With the detailed overview of the XFEM method and Abaqus XFEM capabilities, it can also serve as a complete source for future references. 
Texas Tech University, Jay Sepehri, May 2014

\section{Chapter 2}

\section{Extended Finite Element Method}

\subsection{Introduction}

When using analytical methods in solving fracture mechanics problems, usually there are several limitations which require simplification through some assumptions. Usually homogeneous and isotropic material in an infinite domain with simple boundary conditions is considered to simply the problem.

In Practical problem with complex structures including fracture propagation in rock formations, there are usually very small fractures and subjected to complex boundary conditions and material properties are much more complicated than those related to the ideal linear, homogeneous and isotropic material model. Therefore a more realistic fracture mechanics analysis has to be performed by means of numerical methods. Among these, the most widely adopted in practical engineering applications is finite element method (Zienkiewics, Taylor, and Zhu, 2000). For this reason, several software packages based on the FEM technique have been developed throughout the years.

Although the finite element method has shown to be particularly well-suited for fracture mechanics problems, the non-smooth fracture tip fields in terms of stresses and strains can be captured only by a locally refined mesh. This leads to an increase in number of degrees of elements and simulation run time consequently. Concerning the fracture propagation analysis, it still remains a challenge for industrial modeling application. Since it is required for the FEM discretization to conform the discontinuity, for modeling evolving discontinuities, the mesh has to be regenerated at each time step. This means that the solution has to be projected for each time step on the updated mesh, causing a dramatic rise in terms of computational costs and loss of the quality of results. Because of these limitations, several numerical approaches to analyze fracture mechanics problems have been proposed (Karihaloo and Xiao, 2003).

In recent years, the extended finite element method (XFEM) has emerged as a powerful numerical procedure for the analysis of fracture problems. It has been widely 
acknowledged that the method eases fracture growth modeling under the assumptions of linear elastic fracture mechanics (LEFM). Since the introduction of the method in 1999, many new extensions and applications have appeared in the scientific literature (Karihaloo and Xiao, 2003).

Compared to the finite element method, the X-FEM provides many improvements in the numerical modeling of fracture propagation. In the traditional formulation of the FEM, a fracture is modeled by requiring the fracture to follow element edges. In contrast, the fracture geometry in the X-FEM does not need not to be aligned with the element edges, which is a great flexibility. This capability has been illustrated in the figure 2.1 .
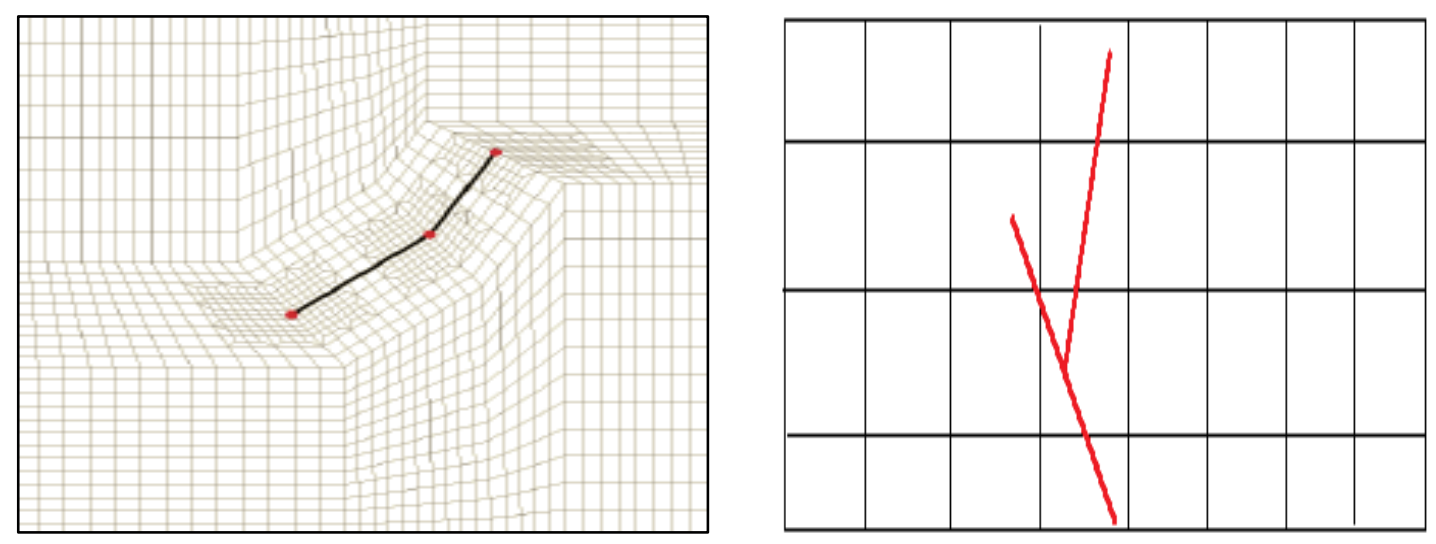

Figure 2.1: Comparison of fracture path in FEM (left) and XFEM ( right)

The XFEM is based on the enrichment of the FE model by adding extra degrees of freedom that are added to the nodes of the elements cut by the fracture. In this way, fracture is included in the numerical model without the need to modify the domain discretization, because the mesh is generated completely independent of the fracture. Therefore, only a single mesh is needed for any fracture length and orientation. In addition, nodes surrounding the fracture tip are enriched with functions that reproduce the asymptotic fracture tip behavior. This enables the modeling of the fracture within the fracture-tip element and increases the accuracy in the computation of the stress intensity factors. 


\subsection{Partition of Unity}

Partition of unity is a set $\mathrm{R}$ of continuous functions from $\mathrm{X}$ to the interval $[0$, 1] such that for every point, $x \in X$,

- there is a neighborhood of $\mathrm{x}$ where all but one finite number of the functions of $\mathrm{R}$ are 0 ,

- $\quad$ the sum of all the function values at $\mathrm{x}$ is $1, \sum_{i=1}^{n} f_{i}(x)=1$

Partitions of unity are useful because they often allow extending local constructions to the whole space. They are also important in the interpolation of data, in signal processing, and the theory of spline functions.(Wikipedia) Example of partition of unity for 4 functions is illustrated in figure 2.2.

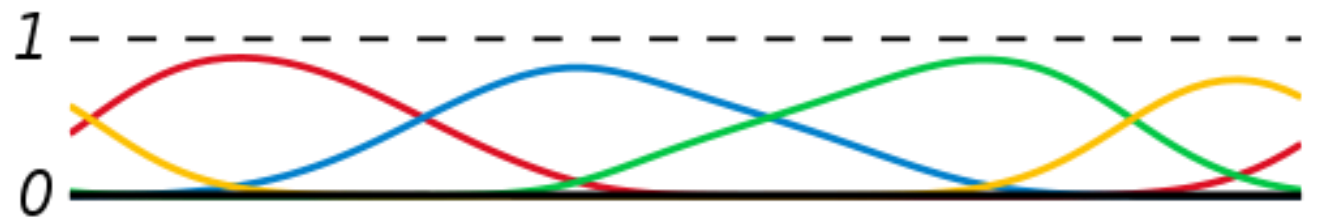

Figure 2.2: Partition of unity of a circle with four functions (Wikipedia)

If this condition is verified, any point $\mathrm{x}$ in $\mathrm{X}$ has only finite $i$ with $f_{i}(x) \neq 0$. It can be shown that, the sum in Equation 1 does not have to be identically unity to work; in fact, for any function $\varphi(\mathrm{x})$, it is verified that

$\sum_{i=1}^{n} f_{i}(x) \varphi(x)=\varphi(x)$

It can also be shown that the partition of unity property is satisfied by the set of finite element shape functions $N j$, i.e.

$\sum_{j=1}^{m} N_{j}(x)=1$

\subsubsection{Partition of Unity Finite Element Method}

To improve a finite element approximation, the enrichment procedure may be applied. In other words, the accuracy of solution can be improved by including the 
analytical solution of the problem in the finite element formulation. For example, in fracture mechanics problems, if the analytical fracture tip solution can be added to the framework of the finite element discretization, predicting fracture tip fields may be improved. This will results in increase in the number of degrees of freedom

The partition of unity finite element method (PUFEM) (Melenek and Babuska, 1996; Gasser and Hozapfel, 2005), using the concept of enrichment functions along with the partition of unity property, can help to obtain the following approximation of the displacement within a finite element.

$u^{h}(x)=\sum_{j=1}^{m} N_{j}(x)\left(u_{j}+\sum_{i=1}^{n} p_{i}(x) a_{j i}\right)$

where, $p_{i}(x)$ are the enrichment functions and $a_{j i}$ are the additional unknowns or degrees of freedom associated to the enriched solution. $m$ and $n$ are the total number of nodes of finite elements and the number of enrichment functions $p_{i}$.

Based on the discussion above, for an enriched node $x_{k}$, equation 3 might be written as:

$u^{h}\left(x_{k}\right)=\left(u_{k}+\sum_{i=1}^{n} p_{i}\left(x_{k}\right) a_{j i}\right)$

which is not a possible solution. To overcome this problem and satisfy interpolation at nodal point, i.e. $\mathrm{u}^{\mathrm{h}}\left(\mathrm{x}_{\mathrm{ki}}\right)=\mathrm{u}_{\mathrm{i}}$, a slightly modified expression for the enriched displacement field was proposed as below

$$
u^{h}(x)=\sum_{j=1}^{m} N_{j}(x)\left[u_{j}+\sum_{i=1}^{n}\left(p_{i}(x)-p_{i}\left(x_{j}\right)\right) a_{j i}\right]
$$

\subsubsection{Generalized Finite Element Method}

A great improvement in finite element discretization is provided by the generalized finite element method (GFEM), in which two separate shape functions are used for the ordinary and for the enriched part of the finite element approximation (Strouboulis and Copps; Duarte, Babuska, and Oden), where 
$u^{h}(x)=\sum_{j=1}^{m} N_{j}(x) u_{j}+\sum_{j=1}^{m} \bar{N}_{j}(x)\left(\sum_{i=1}^{n} p_{i}(x) a_{j i}\right)$

Where $\bar{N}_{j}(x)$ are the shape functions associated with the enrichment basis function $p_{i}(x)$. For the reason explained in previous section, equation 6 should be modified as follows:

$u^{h}(x)=\sum_{j=1}^{m} N_{j}(x) u_{j}+\sum_{j=1}^{m} \bar{N}_{j}(x)\left[\sum_{i=1}^{n}\left(p_{i}(x)-p_{i}\left(x_{j}\right)\right) a_{j i}\right]$

\subsection{Enrichment Functions}

In two-dimensional problems, fracture modeling is characterized using of two different types of enrichment functions:

\subsubsection{The Heaviside Function}

For the elements completely cut by the fracture, The Heaviside function $\mathrm{H}(\mathrm{x})$ is applied for enrichment. The splitting of the element by the fracture results in a jump in the displacement field and the Heaviside function provides a simple mathematical approach to model this kind of behavior.

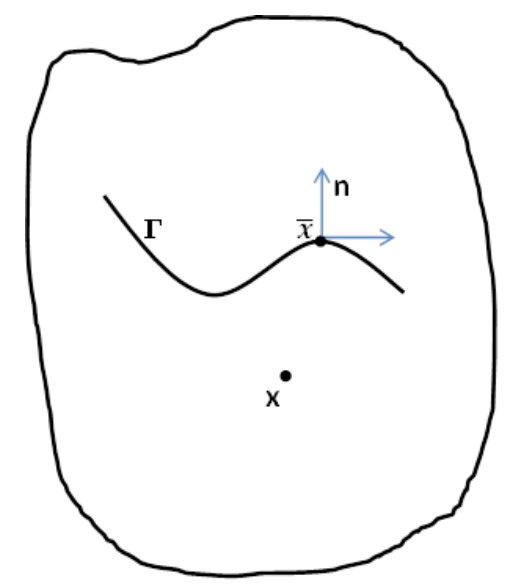

Figure 2.3: Evaluation of Heaviside function

For a continuous curve $\Gamma$, representing a fracture within the deformable body $\Omega$, we can consider a point $x(x, y)$ in $\Omega$ (figure 2.3 ). The objective is to determine the position of this point with respect to the fracture location. If the closest point 
belonging to $\Gamma$ is $\bar{x}(\bar{x}, \bar{y})$ and the outward normal vector to $\Gamma$ in $\bar{x}$ is $\mathbf{n}$, the Heaviside function might be defined as follows

$H(x, y)=\left\{\begin{array}{lll}1 & \text { for } & (x-\bar{x}) \cdot \boldsymbol{n}>\mathbf{0} \\ -1 & \text { for } & (x-\bar{x}) \cdot \boldsymbol{n}<\mathbf{0}\end{array}\right.$

This function introduces the discontinuity across the fracture faces.

\subsubsection{Asymptotic Near-Tip Field Functions}

For those elements that are not completely fractured and containing fracture tip, the Heaviside function cannot be used to approximate the displacement field in the entire element. For the fracture tip, the enrichment functions originally introduced by Fleming (Fleming et al., 1997) for use in the element free Galerkin method. They were later used by Belytschko (Belytschko et al.,1999) for use in XFEM formulation. These four functions describe the fracture tip displacement field. The first function is discontinuous at the fracture tip.

$$
\left[F_{\alpha}(r, \theta), \alpha=1\right]=\left\{\begin{array}{c}
\sqrt{r} \sin \left(\frac{\theta}{2}\right) \\
\sqrt{r} \cos \left(\frac{\theta}{2}\right) \\
\sqrt{r} \sin \left(\frac{\theta}{2}\right) \sin \theta \\
\sqrt{r} \cos \left(\frac{\theta}{2}\right) \sin \theta
\end{array}\right.
$$

In this formulation $r, \theta$ are polar coordinates defined at the fracture tip. The above functions can reproduce the asymptotic mode I and mode II displacement fields in LEFM, which represent the near-tip singular behavior in strains and stresses. These functions significantly improve the accuracy of calculation of $K_{I}$ and $K_{I I}$. (Moës, Dolbow and Belytschko, 1999)

By using the enrichment functions in equation 10, four different additional degrees of freedom in each direction for each node are added to those related to the finite element formulation. The term $\sqrt{r} \sin \left(\frac{\theta}{2}\right)$ is discontinuous and therefore can represent the discontinuous behavior at the fracture tip. The remaining three functions 
are used to enhance approximation of the solution in the neighborhood of the fracture tip.

Figure 2.4 shows a part of a mesh with four-node bilinear types of elements. The circled nodes are the nodes of elements completely cut by the fracture and therefore enriched with Heaviside function. The nodes with green square are containing fracture tip and are enriched by fracture tip special function mentioned in equation 9 .

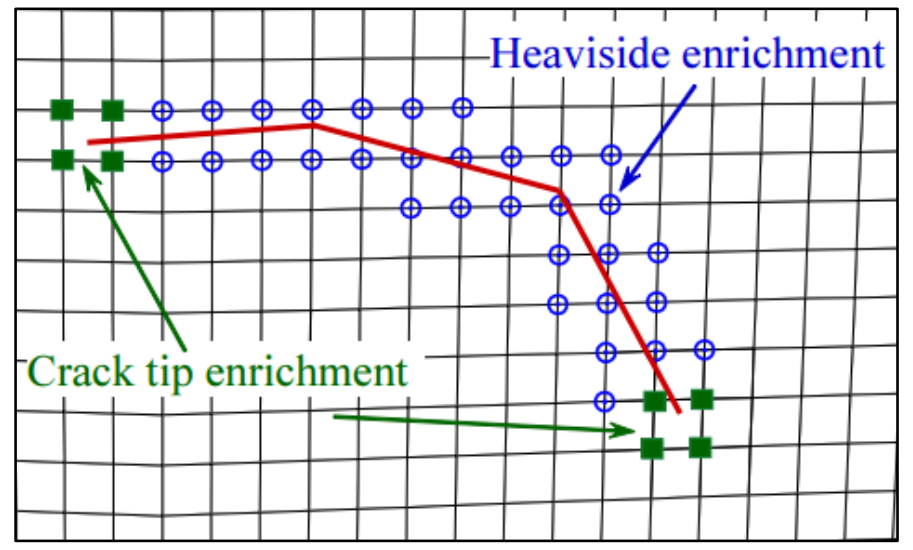

Figure 2.4: Enriched nodes in XFEM (Giner, Sukumar, and Taranc, 2008)

Based on what discussed about the enrichment functions, the following expression for the XFEM approximation might be formulated

$u^{h}=u_{F E M}(x)+u_{E n r}(x)$

$$
\begin{gathered}
u_{x f e m}=\sum_{i \in I} N_{i}(x) u_{i}+\sum_{j \in J} N_{i j}(x)[H(x)] a_{j}+\sum_{k \in K_{I}}\left[N_{k}(x) \sum_{l=1}^{4} b_{k}^{l I} F_{l}^{I}(x)\right] \\
+\sum_{k \in K_{I I}}\left[N_{k}(x) \sum_{l=1}^{4} b_{k}^{l I I} F_{l}^{I I}(x)\right]
\end{gathered}
$$

where, $J$ is the set of nodes whose elements is completely cut by the fracture and therefore enriched with the Heaviside function $H(x), K_{I}$ and $K_{I I}$ are the sets of nodes containing the fracture tips 1 and 2 and fracture tip enrichment functions are $F_{l}^{I}(x)$ 
and $F_{l}^{I I}(x) . u_{i}$ are the standard degrees of freedom, and $a_{j}, b_{k}^{l I}, b_{k}^{l I I}$ are the vectors of additional nodal degrees of freedom for modeling fracture faces and the two fracture tips, respectively.

\subsection{Level Set Method for Modeling Discontinuities}

In some cases numerical simulations include moving objects, such as curves and surfaces on a fixed grid. This kind of modeling and tracking is difficult and requires complex mathematical procedure. The Level Set Method (LSM) is a numerical technique that can help solving these difficulties. The key point in this method is to represent moving object as a zero level set function. To fully characterize a fracture, two different level set functions are defined:

1. A normal function, $\varphi(x)$

2. A tangential function, $\psi(x)$.

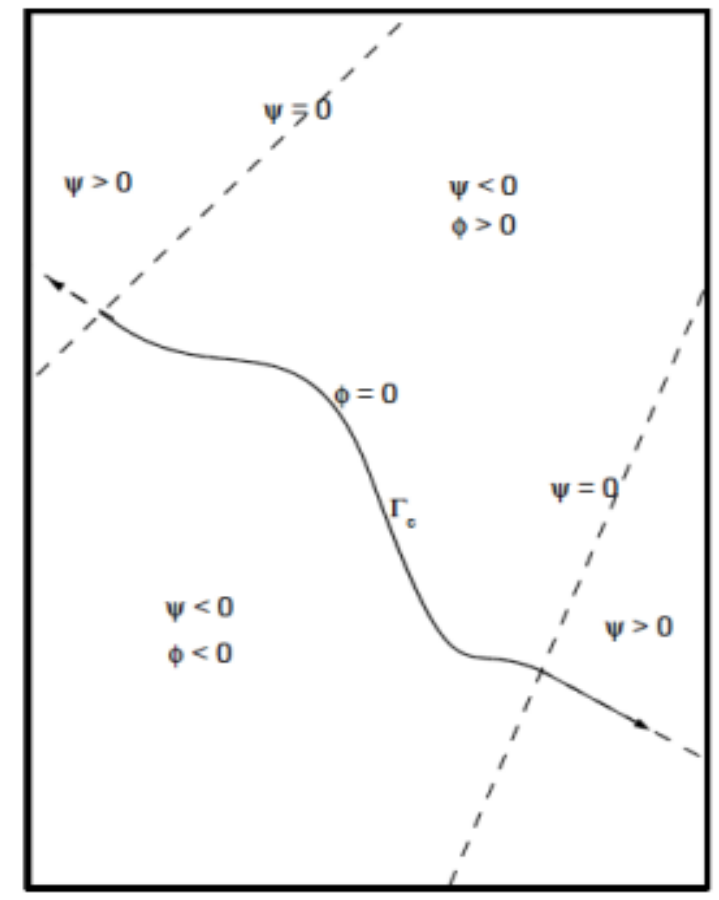

Figure 2.5: Construction of level set functions

For the evaluation of the signed distance functions, assume $\Gamma_{c}$ be the fracture surface (shown on figure 2.5) and $\mathrm{x}$ the point we want to evaluate the $\varphi(x)$ function. The normal level set function can be defined as 
$\varphi=(x-\bar{x}) \cdot n$

Where $\bar{x}$ and $n$ are defined previously.

In figure 2.6 the plot of the normal signed function $\varphi(x)$ for a fracture is illustrated. The tangential level set function $\psi(\mathrm{x})$ is computed by finding the minimum signed distance to the normal at the fracture tip. In case of an interior fracture, two different functions can be applied. However, a unique tangential level set function can be defined as

$\psi(x)=\max \left(\psi_{1}(x), \psi_{2}(x)\right)$

In conclusion, referring to Figure 2.5, it may be written as follows:

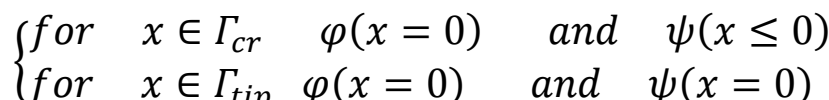

where $\Gamma_{\text {tip }}$ indicates the fracture tips location.

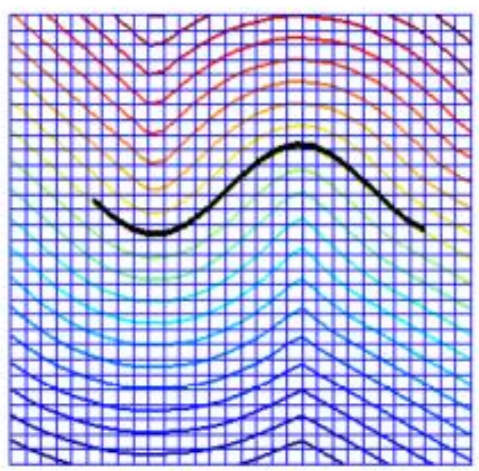

(a) $2 \mathrm{D}$ contour of $\varphi(\mathbf{x})$.

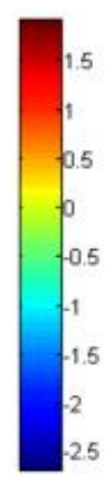

Figure 2.6: Normal level set function $\varphi(x)$ for an interior crack

\subsection{Numerical Integration and Convergence}

The Gauss quadrature has been used for polynomial integrands. For nonpolynomial ones, this method may reduce the accuracy of results. Introducing an a fracture in the finite element discretization changes displacements and stresses into non-linear fields which cannot be integrated by the Gauss quadrature. 
To solve this problems, a subtriangulation procedure was proposed (Dolbow, 1999), in which elements edges align with the fracture faces. This approach is illustrated in figure 2.7. Within these sub-elements the standard Gauss integration procedure can be used.

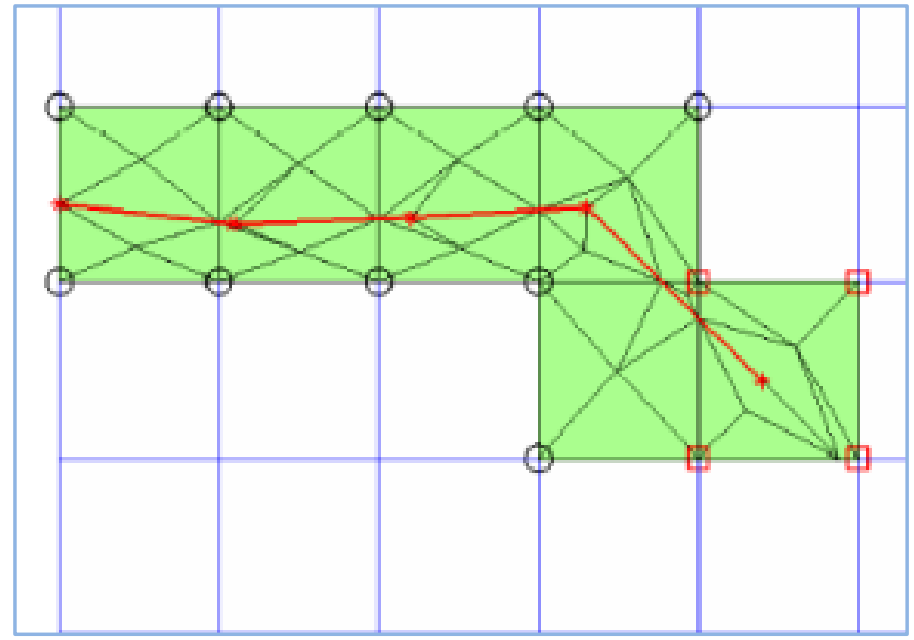

Figure 2.7: Subtriangulation of elements cut by a fracture

For elements containing the fracture tip, therefore including the singular stress field, this procedure might result to be inaccurate if Gauss points of subtriangles are too close at the fracture tip.

Fracture modeling with the standard finite element method is performed by remeshing the domain so that elements boundaries match the fracture geometry. But new created elements have to be well conditioned and not badly shaped. Accordingly, remeshing procedure is a complicated and computationally costly operation. On the other hand, since the subtriangulation is performed only for integration purposes, no additional degrees of freedom are added to the system and subtriangles are not forced to be well shaped.

An alternative method based on the elimination of quadrature sub-elements has been proposed (Ventura, 2006). In such approach, rather than partitioning elements cut by a fracture, discontinuous non-differentiable functions are replaced with equivalent polynomial functions and consequently the Gauss quadrature can be carried out over the whole element. 
The XFEM method provides more accurate results than FEM one when there are discontinuity included in the domain. But, it cannot improve the rate of convergence due to the presence of the singularity and the convergence is lower than what expected by using the FEM method in smooth problems. Several methods have been proposed during last decades to achieve an optimal rate of convergence, e.g. XFEM with a fixed enrichment area, high-order XFEM (Laborde et al. 2005; Xiao and Karihaloo, 2006) as well as a modified construction of blending elements.

\subsection{XFEM Implementation in Abaqus}

The first formulation of the XFEM goes back to the 1999 and therefore there is a shortage of commercial software that have used such method. The enormous improvements provided by the XFEM, have made many to attempt to include XFEM in multi-purpose commercial FEM software. Among the commercial software, the most famous ones are LS-DYNA and Abaqus and ANSYS. There are also other software including ASTER and Morfeo which have included this capability

XFEM module was introduced for the first time in Abaqus in 2009 with the Abaqus 6.9 release (Dassault Systemes, 2009). The XFEM implementation in Abaqus/Standard is based on the phantom nodes method (Song, Areias, and Belytsckho, 2006) in which phantom nodes are superposed to the real ones to reproduce the presence of the discontinuity.

The fracture surfaces and the fracture tip location in Abaqus are identified with a numerical procedure based on the Level Set Method. Once the mesh discretization has been created, each node of the finite element grid is characterized with its three coordinates with respect to the global coordinate system and two additional parameters, called PHILSM and PSILSM. These parameters are nonzero only for the enriched elements and they might be easily interpreted as the nodal coordinates of the enriched nodes in a coordinate system centered at the fracture tip and whose axes are, respectively, tangent and normal to the fracture surfaces at the fracture tip. (Dassault Systemes, 2009) 


\subsubsection{Modeling Approach}

The extended finite element method (XFEM) has solved the shortcomings associated with meshing fracture surfaces. The presence of discontinuities is simulated by the special enriched functions and additional degrees of freedom. For the purpose of fracture analysis, the enrichment functions typically consist of the near-tip asymptotic functions that represent the singularity around the fracture tip and a Heaviside function that simulate the jump in displacement across the fracture surfaces. Modeling the fracture-tip singularity requires that fracture being constantly tracked. This is a very complex procedure because the degree of fracture singularity depends on the location of the fracture. Therefore, the asymptotic singularity functions are only considered when modeling stationary cracks in Abaqus/Standard (Dassault Systemes, 2009).

Two kinds of approach are used to study moving fractures. The first one uses the concept of Fracture Mechanics and fracture growth is predicted when a combination of the components of the energy release rate is equal to, or greater than, a critical value. Techniques such as virtual crack closure technique (VCCT), J-integral method and virtual crack extension have often been used to calculate the ERR. The second one is developed based on Damage Mechanics and uses cohesive zone concept in which the interface containing the fracture is modeled by a damageable material. Then, fracture is initiated when a damage criterion reaches its maximum value. (Burlayenko and Sadowski, 2008)In Abaqus, Cohesive segment method and VCCT technique are used in combination with phantom node technique to model moving fracture.

\subsubsection{The Cohesive Segments Method and Phantom Nodes}

This approach is used in Abaqus/Standard to simulate fracture initiation and propagation and is based on cohesive elements or on surface-based cohesive behavior. In this method, the cohesive surfaces must be aligned with element boundaries and the fractures propagate along a known path within cohesive material. But using the XFEM-based cohesive method in Abaqus, fracture initiation and propagation can be 
simulated along an arbitrary path in the materials (Dassault Systemes, 2009). Using this approach the near-tip singularity is not required and only the displacement jump function across an element is considered. Therefore, the fracture has to propagate across an entire element at a time to avoid the need to model the stress singularity.

Phantom nodes, are imaginary nodes superposed on the original nodes to simulate the discontinuity of the across the fracture in an elements cut by fracture. This concept is illustrated graphically in Figure 2.8. For intact elements, each phantom node is constrained to its corresponding real node but when element is cut by a fracture, the cracked element is divided into two separate parts. Each part has a combination of real and phantom nodes. Therefore phantom nodes and their corresponding real nodes can move independently (Xia, Du, and Wohlever, 2012).

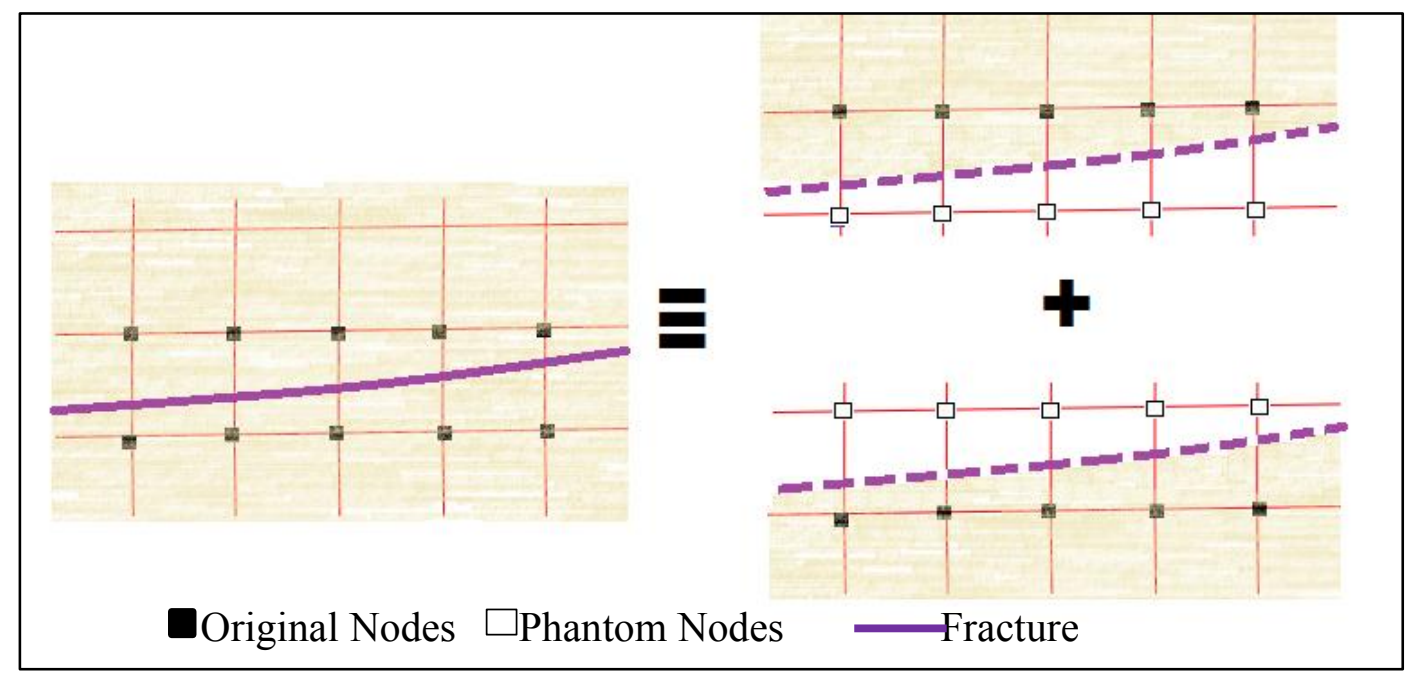

Figure 2.8: The principle of the phantom node method (Dassault Systemes, 2009)

\subsubsection{The Principles of LEFM and Phantom Nodes}

This method is more appropriate for fracture propagation problems in brittle materials. In this method, also, only the displacement jump function in cracked element is considered and the fracture has to propagate the entire element at once to avoid the need to model the stress singularity. The strain energy release rate at the fracture tip is calculated based on the modified Virtual Crack Closure Technique (VCCT). Using this approach fracture propagation along an arbitrary path can be simulated without the need to fracture path being known a priori. 
The modeling technique is similar to the XFEM-based cohesive segment approach. In this method also phantom nodes are introduced to represent the discontinuity of the enriched elements. The fracture criterion is satisfied when the equivalent strain energy release rate exceeds the critical strain energy release rate at the fracture tip in the enriched element.

\subsubsection{Virtual Crack Closure Technique}

Fracture propagation phenomena can be similar to separation of anisotropic layers. Therefore, fracture mechanics principles (Janssen, Zuidema, and Wanhill, 2004) can be used to study the behavior of fracture and to determine the conditions for the fracture initiation and growth. The fracture propagation is possible when the energy released for unit width and length of fracture surface (called Strain Energy Release Rate, $G$ ) is equal to a critical level or fracture toughness which is material property (Janssen, Zuidema, and Wanhill, 2004).

Virtual Crack Closure Technique is based on the assumption that for a very small fracture opening, the strain energy released is equal to the amount of the work required to close the fracture. The work $W$ required to close the fracture can be evaluated by evaluating the stress field at the fracture tip for a fracture of length $a$, and then obtaining displacements when the fracture front extended from $a$ to $a+\Delta a$ as illustrated in figure 2.9.

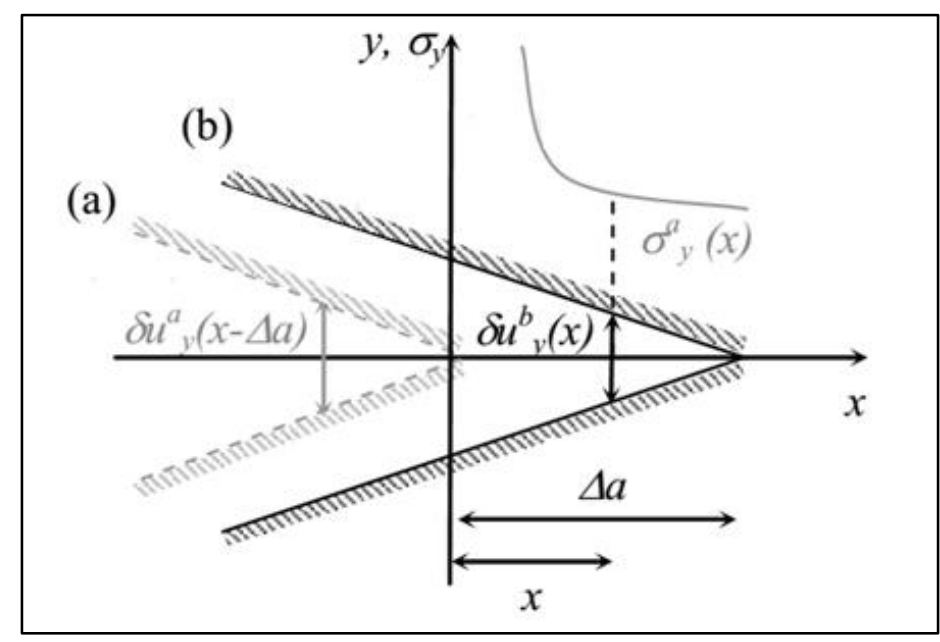

Figure 2.9: Illustration of fracture extension in VCCT(Elisa, 2011) 
The expression of the work $W$ based on this two-steps Virtual Crack Closure Technique is given by (Elisa, 2011)

$$
W=\frac{1}{2}\left(\int_{0}^{\Delta a} \sigma_{y y}^{(a)}(x) \delta u_{y}^{(b)}(x) d x+\int_{0}^{\Delta a} \sigma_{y x}^{(a)}(x) \delta u_{x}^{(b)}(x) d x+\int_{0}^{\Delta a} \sigma_{y z}^{(a)}(x) \delta u_{z}^{(b)}(x) d x\right)
$$

Another approach which is the one step Virtual Crack Closure Technique is based on the assumption that a very small fracture extension has negligible effects on the fracture front. In this case, stress and displacement can be calculated in the same step by performing one analysis. Using this technique, the expression of the work $W$ needed to close the fracture becomes:

$$
\begin{gathered}
W=\frac{1}{2}\left(\int_{0}^{\Delta a} \sigma_{y y}^{(a)}(x) \delta u_{y}^{(b)}(x-\Delta a) d x+\int_{0}^{\Delta a} \sigma_{y x}^{(a)}(x) \delta u_{x}^{(b)}(x-\Delta a) d x\right. \\
\left.+\int_{0}^{\Delta a} \sigma_{y z}^{(a)}(x) \delta u_{z}^{(b)}(x-\Delta a) d x\right)
\end{gathered}
$$

where both displacements and stress are evaluated in the step (a) of Figure 2.9. Based on the definitions given, the Energy Release Rate can be written as

$$
G=\lim _{\Delta a \rightarrow 0} \frac{W}{\Delta a}
$$

\subsubsection{Fracture Growth Criteria}

In fracture mechanics, the Strain Energy Release Rate $(G)$ is compared with the material fracture toughness $\left(G_{C}\right)$, as the criterion for the fracture initiation and propagation. When $G$ is larger than $G_{C}$, fracture initiates $\left(G>G_{C}\right)$. Experimental tests are usually used to measure $G_{C}$. But, for an accurate and comprehensive test, several different types of samples are needed to generate fracture toughness data over a desired range of mixed-mode combinations (Reeder and Crews, 1990). Therefore, several empirical criteria have been offered to calculate fracture toughness for mixed mode. One of the most used criteria is the power law which may be used to represent a 
wide range of material responses by selecting the two exponents, $\alpha$ and $\beta$. Power law criterion is expressed as

$\left(\frac{G_{I}}{G_{I C}}\right)^{\alpha}+\left(\frac{G_{I I}}{G_{I I C}}\right)^{\alpha \beta}+\left(\frac{G_{I I I}}{G_{I I I C}}\right)^{\gamma}=1$

Another one is the B-K criterion (Benzeggagh and Kenane, 1996), which requires the selection of only one fitting parameter $\eta$. The $\mathrm{B}-\mathrm{K}$ criterion is

$\frac{G_{T}}{G_{I C}+\left[\left(G_{I I C}-G_{I C}\right) \frac{G_{I I}}{G_{T}}+\left(G_{I I I C}-G_{I C}\right) \frac{G_{I I I}}{G_{T}}\left(\frac{G_{I I}+G_{I I I}}{G_{T}}\right)^{\eta-1}\right]}=1$

These criteria are empirical mathematical expressions to represent different material responses by varying the values assigned to the fitting parameters. Thus, the selection of these parameters requires that mixed-mode testing be performed during the characterization of the material(Elisa, 2011). 


\section{Chapter 3 \\ Perforations Design}

\subsection{Introduction}

Hydraulic fracturing has been used extensively as a successful stimulation method to improve production from oil and gas reservoirs. Understanding fracture initiation and propagation from wellbores is essential in performing efficient hydraulic fracture stimulation treatment. Because of well stability problems, isolating well from unwanted zones and several other operational considerations, usually wellbores are cased and then perforated; therefore, majority of hydraulic fracturing treatments are performed through perforations.

Perforating provides the channel of communication between the wellbore and the reservoir. In hydraulic fracture treatment, perforation may serve as an initial fracture to help with fracture initiation and control propagation direction. Perforations play an important role in the complex fracture geometries around wellbore. Initiation of a single wide fracture from a wellbore is one of the main objectives of using the perforation as a means to avoid multiple T-shaped and reoriented fractures.

Different parameters contribute to the success of stimulation treatment through perforation. In the following section, after reviewing the wellbore perforation and highlighting the parameters in perforation design, the role of perforation in hydraulic fracturing is discussed.

\subsection{Parameters in Designing Perforation}

The last step of the completion will be to run perforating guns, a string of shaped charges, down to the desired depth and firing them to perforate the casing. Perforations from shaped charges create channels of usually less than 0.8 inch diameter at the entrance hole in the casing and depth of 1 to 30 inches (PetroWiki, 2014). Flow behavior from perforation is dominated by radial flow with some pseudo-radial character in longer perforations. Length, diameter, and permeability of the rock around the perforation 
control flow through a perforation. (Locke, 1981) figure 3.1 illustrates perforation parameters.

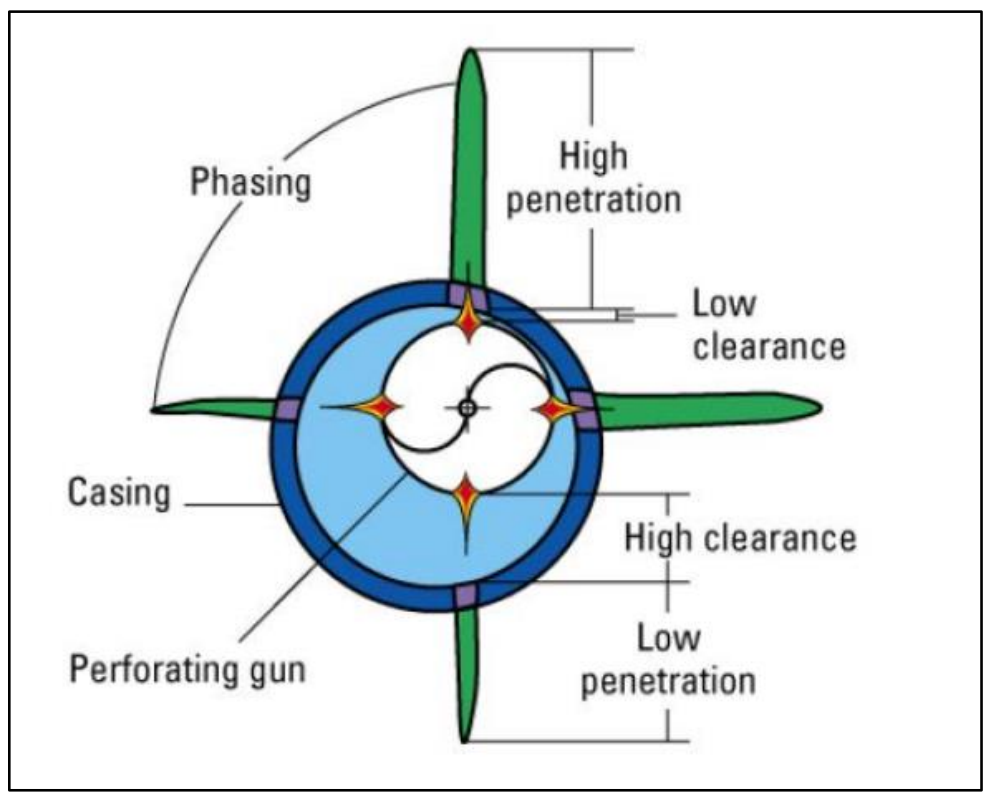

Figure 6.1: Schematic of Perforation gun and parameters

Length of the perforation is one of the most critical factors in completions which no further stimulation or sand control is planned. The effect of perforation diameter becomes more important when sand control completion designs are planned or fracturing is needed. These two factors are less important when the effect of formation damage is included.

As the shape change penetrates the formation, the material is pushed to the sides, resulting in a zone of lowered permeability. The amount of permeability loss depends on the porosity, formation fluid and size and design of the shape charge. Permeability loss in relatively homogeneous formations can be approximately 35 to 80 percent of the initial formation permeability. To achieve a highly conductive perforation, optimum perforating equipment, appropriate charge and application method (underbalance, overbalance, surging, etc.) must be selected carefully to minimize formation damage and provide the best cleanup and flow capacity in the perforations (PetroWiki, 2014).

The best-known operational design considerations for perforating are:

- Perforation phasing (Shot-phase angle) 
- Perforation density (Shot per foot)

- Perforation length

- Perforation diameter

- Stimulation type

- Well deviation angle

These affecting parameters are discussed in the following subsections:

\subsubsection{Perforation Phasing}

Phasing is the angle between the two consecutive perforations. Figure 3.2 shows common perforation phasing angles. Although there are many possible angles, the five common values applied are $0^{\circ}, 180^{\circ}, 120^{\circ}, 90^{\circ}$ and $60^{\circ}$. In the $0^{\circ}$-phasing, all the perforations are in a row and this phasing is used only in the smaller outside diameter guns or guns in very large casing. $0^{\circ}$ phasing has some issues because applying all the shots in a row reduces casing yield strength and makes it more vulnerable to splits and collapse.

Perforating phasing is known to affect production. For example, for a 12-in. penetration into the formation, a theoretical productivity ratio of 1.2 is predicted from $90^{\circ}$ phasing of $4 \mathrm{SPF}$, while the productivity ratio is approximately 0.99 when the 4 shots are in $0^{\circ}$ phasing. (Locke, 1981)

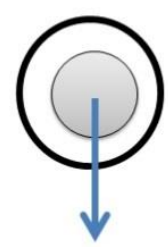

$0^{\circ}$

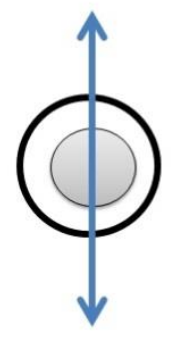

$180^{\circ}$

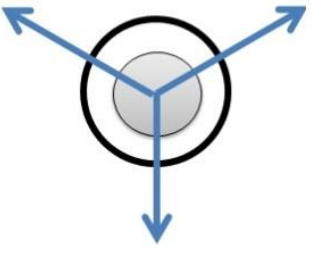

$120^{\circ}$

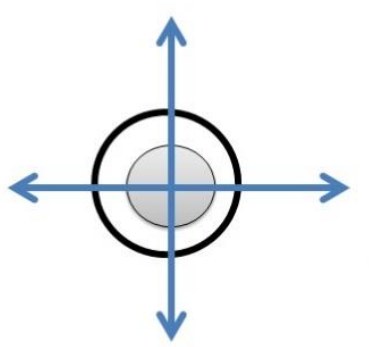

$90^{\circ}$

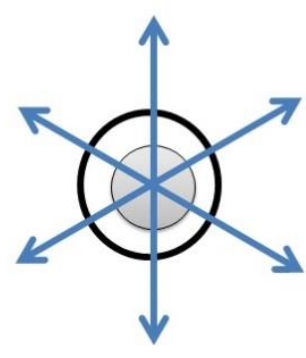

$60^{\circ}$

Figure 6.2: Common perforation Phasing angles 


\subsubsection{Perforations Density}

The density of perforations (measured in shot per foot or meter, SPF or SPM respectively) is always an important factor in completion design. Shot densities from 1 to 27 SPF (3 to 88 SPM) may be applied. For formations with high flow rates, large densities are usually applied. Also for some cases including single point application of fractures in deviated wellbores and for layered formations, increasing number of shot can be very effective.

Assuming all perforations are active and open to flow, perforation densities of 4 SPF with $90^{\circ}$ phasing and with 0.5 -in. holes usually creates the equivalent of openhole productivity. However, increased shot densities (greater than 4 SPF) may improve productivity ratios for very high flow rate wells or in gravel-packed wells. Usually only approximately half of the total perforations are open perforations that can be effective in producing or injecting fluid. The reason for deactive perforations is usually nonproductive layers in the formation or damaged perforations. Perforating produces a damaged zone around the perforation in which permeability may be highly reduced. Longer perforations are less influenced by the crush zone than short perforations. Phased perforations, such as $90^{\circ}$ phased perforations, might be less affected than $0^{\circ}$ phased perforations. The damage in the near wellbore and in the crushed zone can cause severe pressure drops (PetroWiki, 2014).

\subsubsection{Perforation Length}

Perforation length usually is thought to be the most important characteristic in a perforation design. But, there are several situations in which perforated length does not make a significant difference in well productivity. Only in completions which do not require further stimulation, the perforation length dominates the other factors. In cases such as hydraulic fracturing or pre-packed gravel-pack operations, long perforations may not be an advantage. For hydraulic fracturing or gravel-pack treatments, a large, effective entrance hole through the pipe and cement is more important than total perforation length. 


\subsubsection{Perforation Diameter}

Perforation diameter also may influence the productivity ratio, especially in high productivity wells. Perforation diameter is dependent on shape charge design and the clearance of the gun in the casing. In cases such as sand control operations, unstable formations, and wells that are to be hydraulically fractured, the perforation diameter is important enough to be dominant.

\subsubsection{Stimulation Type}

The type of stimulation or well completion influences the perforation design. In gravel-pack stimulation, a large density, large diameter and low phasing can enhance gravel placement and reduce the fluids velocity into the wellbore (Venkitaraman, Behrmann, and Chow, 2000). Successful gravel placement requires leak-off, which can be achieved by high efficiency perforation. By reducing production fluid velocity less fines will move and plug the pack. Larger number of perforations is needed to generate the same productivity as open perforations, because perforations may be filled with gravel.

Fracturing stimulations also require special perforating design. Perforation number and configuration must be in way to avoid fluid shearing effect (lowering the viscosity by degrading the polymer or crosslink system) and to avoid high pumping costs (Behrmann and Nolte, 1999).

\subsubsection{Well Deviation Angle}

A cased and cemented and highly deviated well may require a completely different perforation design than a vertical well, even in a similar formation. The main factors to be considered for deviated wells are:

- Placement of guns

- Cost of perforating in very long sections

- Need to produce selectively from a certain section of the wellbore

- Coning control

- Need for focusing injected fluid into a single interval when fracturing or acidizing 
Since cost is one of defining parameters in perforation design, using logging tool can help identifying the productive zone of best porosity, oil saturation, and pressure Perforating costs may increase as pay contact increases, leading to reduced perforation and concentrate perforations in those areas.

\subsection{Perforating for Hydraulic Fracturing Treatment}

The main reasons for casing and perforating the well before hydraulic fracturing can be initiation of a single wide fracture from the wellbore, having control over fracture propagation direction, using perforation as onset of fracture and reducing breakdown pressure. Hydraulic fracture treatment calls for specially designed type of perforation.

The objective in perforating design for fracturing should be to choose perforating parameters that minimize near-wellbore pressure drops during both the fracturing operation and production. Some of these near wellbore effects are perforation friction, micro-annulus pinch points from gun phasing misalignment, multiple competing fractures and fracture tortuosity caused by a curved fracture path (Romero, Mack, and Elbel, 1995)

An optimal perforation for fracture propagation would have a minimum injection pressure, initiate only a single fracture and generate a fracture with minimum tortuosity at an achievable fracture initiation pressure (Behrmann and Nolte, 1999).

If fracture treatment is performed in wells with $0^{\circ}$ phasing, there is higher probability of screenout than for the wells with $60^{\circ}, 90^{\circ}$, or $120^{\circ}$ phasing. This can be due to screenout from the smaller diameter perforations or because of one wing of the fracture going around the pipe in the micro-annulus fractures. Phasings of $60^{\circ}, 90^{\circ}$, and $120^{\circ}$ are usually the most efficient options for hydraulic fracture treatment because they will produce a perforation just with few degrees from any possible fracture direction.

In hydraulic fracturing treatment in deviated wells we need to decide on whether to perforate the whole zone or to concentrate the perforations in a specific zone to ensure a single fracture propagating. Concentrating perforations can control the point of fracture initiation. Field performance has indicated that perforating at 8 to $16 \mathrm{SPF}$ over a $2-$ to 5 -ft interval is sufficient to initiate a fracture. 
In addition to parameters discussed in previous section, some of extra considerations when designing perforation for hydraulic fracturing treatment are discussed in the following sections.

\subsubsection{Minimum and Maximum Horizontal Stress Direction}

Maximum and minimum horizontal stresses and vertical stress from overburden describe in-situ stress conditions in oil and gas reservoirs. Hydraulic fractures initiate and propagate along a preferred fracture plane (PFP), which is the path of least resistance. In most cases, stress is greatest in the vertical direction, so the PFP is horizontal and is parallel to the maximum horizontal stress.

Perforations that are not aligned with the PFP create complex flow paths near wellbore during hydraulic fracturing treatments. This effect called tortuosity is illustrated in figure 3.3. Tortuosity causes additional pressure drops that increase pumping pressure needed and limits fracture width, which can result in early screenout from proppant bridging and, eventually, not optimal stimulation treatments (Almaguer et al., 2002).

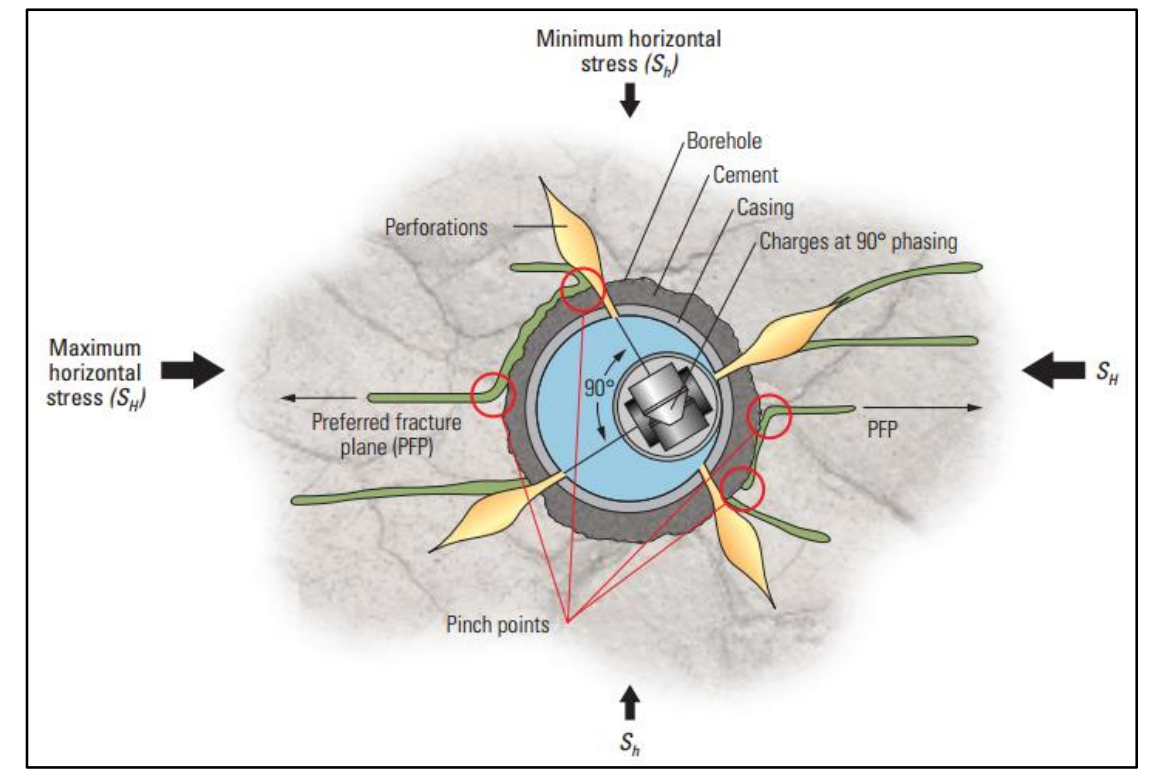

Figure 6.3: Complex fracture geometry around oriented perforation (Almaguer et al., 2002)

When stress concentrations near the wellbore exceed formation tensile strength, borehole breakout may occur. In this case the borehole elongates in the direction of minimum horizontal stress $\left(\mathrm{S}_{\mathrm{h}}\right)$, which is $90^{\circ}$ from the PFP. 
Various openhole logging tools can help determine stress directions prior to perforating. The Dipole Shear Sonic Imager tool (DSI), determines PFP orientation by detecting shear-wave anisotropy, which often results from differences in maximum and minimum horizontal stress directions.

In conductive water-base fluids, the Fullbore Formation MicroImager (FMI) tool generates a circumferential electrical image of the borehole wall and provides quantitative information for analysis of fractures. Engineers use this tool to visualize drilling-induced fractures and borehole breakouts, and to establish their orientation (Serra, 1989).

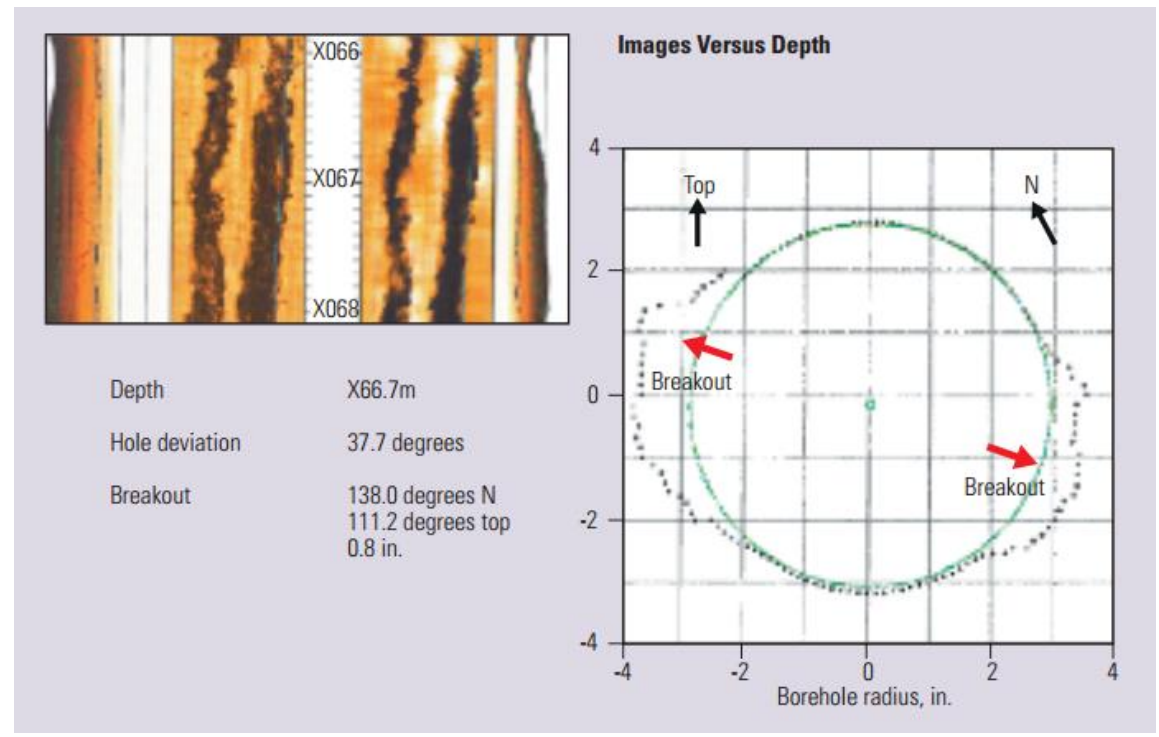

Figure 6.4: Ultrasonic Borehole Imager tool can detect direction of wellbore breakout (Almaguer et al., 2002)

UBI Ultrasonic Borehole Imager tool (figure 3.4) provides circumferential borehole images by generating acoustic images and can be run in nonconductive oil-base fluids to characterize drilling-induced fractures and borehole breakout. Oriented four-arm caliper surveys also provide an indication of borehole breakout, but do not offer circumferential borehole coverage like the DSI, FMI and UBI logging tools. The GeoVision Resistivity tool (GVR ) provides complete circumferential borehole-resistivity images while drilling with conductive fluids (Bonner et al., 1994). 


\subsubsection{Pressure Drop in Perforation}

A pressure drop occurs because of frictional loss at perforation entrance. Calculation of this pressure loss is complicated and depends upon empirical sources such as laboratory data, therefore, in calculating Bottom Hole Treating Pressure (BHTP), this frictional loss is sometimes assumed to be zero or negligible. The friction pressure drop across the perforations is generally expressed by the following equation.

$\Delta P_{\text {perf }}=\frac{0.2369 q^{2} \rho}{N_{p}^{2} d_{p}^{4} C_{d}^{2}}$

where

$\Delta p_{\text {perf }}=$ perforations friction pressure loss, $\mathrm{psi}$,

$q=$ total flow rate, $\mathrm{bbl} / \mathrm{min}$,

$\rho=$ fluid density, lb/gal,

$N_{p}=$ number of perforations,

$d_{p}=$ initial perforation diameter, in., and

$C_{d}=$ coefficient of discharge.

In this equation, there is a term for kinetic energy correction factor, which is known as the coefficient of discharge $\left(C_{d i}\right)$ and is the ratio of the diameter of the fluid stream at the point of lowest pressure drop to the diameter of the perforation as shown in Figure 3.5.

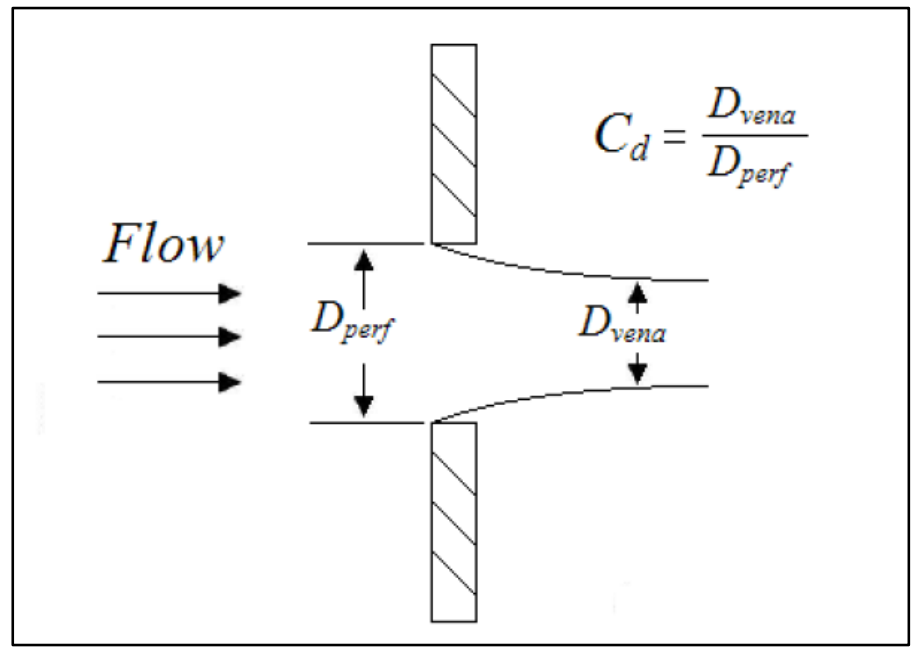

Figure 6.5: Flow through perforations 
It is difficult to estimate perforation friction pressure since the coefficient discharge changes due to perforation erosion and it cannot be determined unless the exact cross-sectional area is known, while the other parameters are easily obtained by real-time field data such as the fluid flow rate and the fluid density.

\subsubsection{Perforation Orientation}

Fracture tends to propagate in a plane of least resistance which is usually called Preferred Fracture Plane (PFP) and is perpendicular to minimum horizontal stress. If the perforation orientation is out of the direction of PFP, induced fracture may initiate along the perforation and as it propagates away from the near wellbore toward the unaltered insitu state of stress, will reorient itself to be perpendicular to the minimum horizontal stress. The farther the perforation angle is off the preferred orientation, the more severe the near-wellbore turning is. Figure 3.6 shows the graphical representation of wellbore, perforation and stresses.

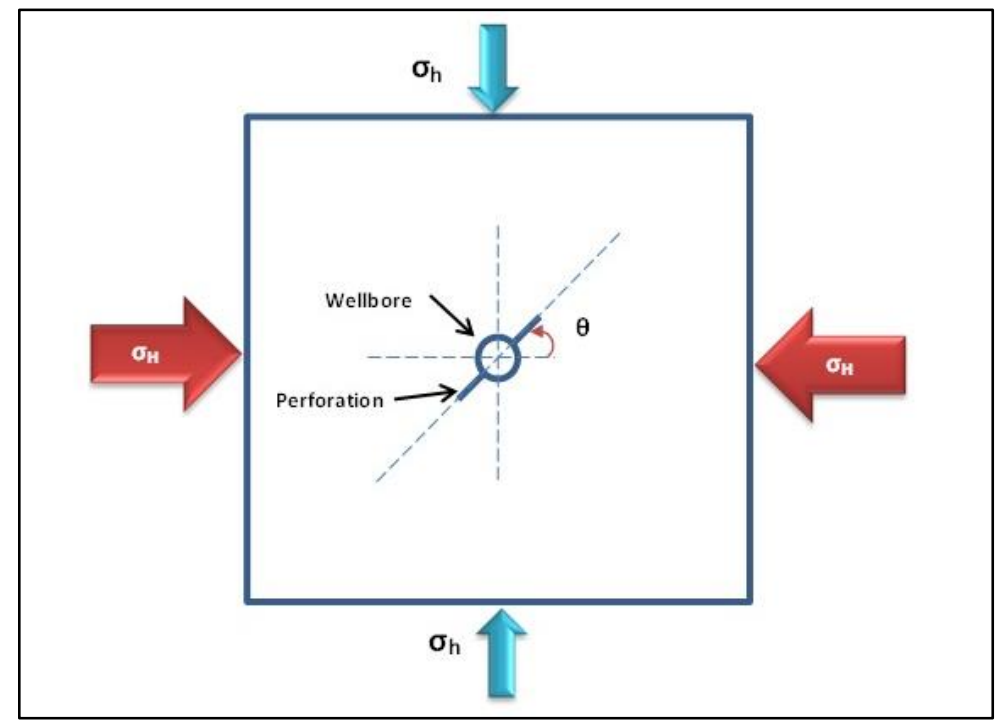

Figure 6.6: Graphical representation of wellbore, perforations and stresses

Experiments show that $180^{\circ}$ phased perforations oriented within 30 degree of the preferred fracture plane (PFP) provide good communication between the perforations and the fracture (Abass et al., 1994). The good connection minimizes the tortuosity. As the perforation angle increases, the fracture breakdown pressure increases because of the horizontal stress difference. Also, when the fracture initiates at the perforations, it must 
turn to eventually align with the PFP. Experiments suggest that a tortuosity effect becomes more pronounced when $\theta$ is 45 degrees or larger. (Abass et al., 1994)

It is obvious that there is a trend of increasing breakdown pressure as the orientation angle relative to the maximum horizontal stress increases. Fracture extension pressure is also a function of the perforation orientation angle.

Perforation orientation can significantly affect the geometry of the fracture initiated from wellbore. For vertical wells, it is recommended to align the perforations in the direction of maximum horizontal stress. Therefore, perforations should be positioned in the PFP, and only 180 degree phasing is required. Those perforations not aligned with the PFP can result in near-well fracture reorientation. Fracture tortuosity can lead to proppant bridging and premature screen-outs. Even if propagated successfully, tortuous fractures are likely to be choked with considerable reduction in the post-treatment production performance (M. Chen et al., 2010).

\subsubsection{Fracture Tortuosity}

If the perforations are misaligned with the direction of PFP, fracture will reorient to propagate in that direction. This will result in a tortuous path of smaller width and rough walls that affect the hydraulic fracture treatment and consequent productivity. Tortuosity is also one of the main reasons for high treatment pressure and proppant blockage and wellbore screenout. Several studies (Cleary et al., 1993; Aud et al., 1994; Romero, Mack, and Elbel, 1995) have identified tortuosity as an important phenomenon that could affect the execution of a hydraulic fracturing treatment.

Tortuosity pressure is defined as the pressure loss of the fracturing fluid as it passes through a region of restricted flow between the perforations and the main fractures. Near-wellbore pressure loss due to fracture tortuosity results from the complicated fracture geometry surrounding the wellbore since the region is usually composed of a complex pathway connecting the wellbore with the main body of the fracture. Figure 3.7 shows a schematic of near-wellbore fracture tortuosity. 


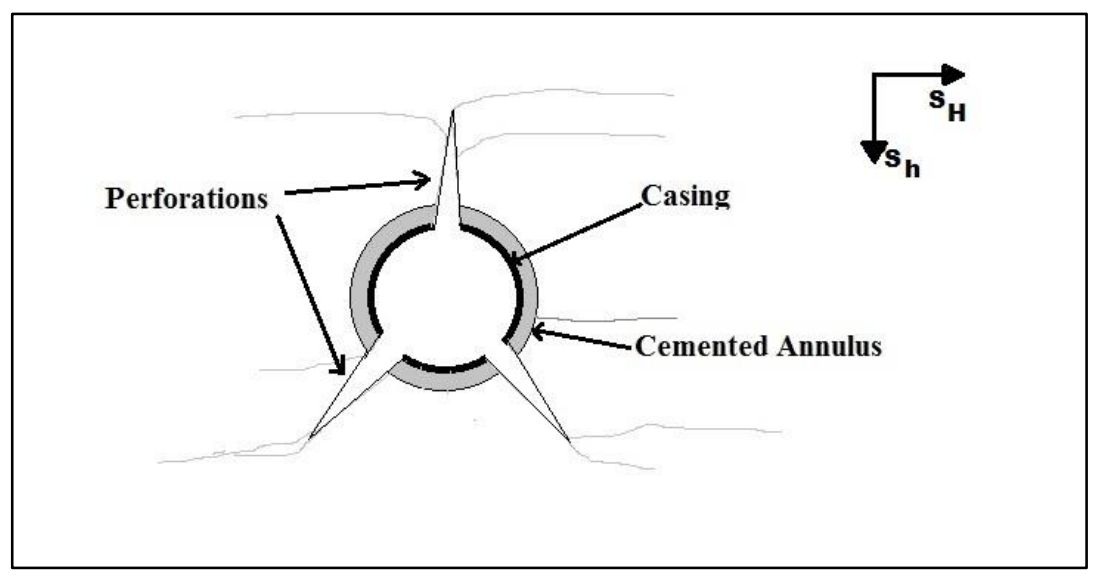

Figure 6.7: A schematic of near-wellbore tortuosity (Wright et al., 1995)

Perforation pressure drop is constant until proppant enters the perforations. Perforation erosion then occurs, which decreases the perforation pressure. Tortuosity friction is largest at the beginning of a treatment, and decreases during the treatment, even without proppant. Perforation misalignment pressure drop can increase as the treatment proceeds if little or no erosion occurs. The erosion can occur with proppant, and possibly even with clean fluid. (Romero, Mack, and Elbel, 1995)

\subsubsection{Micro-Annulus Fracture}

Experimental results indicate that there exists a critical radius about the maximum horizontal stress direction (0-60), in which hydraulic fractures will initiate from the tip of preexisting perforations. For perforations oriented outside this range, hydraulic fractures will initiate from the micro-annulus fractures in the maximum horizontal stress direction (M. Chen et al., 2010).

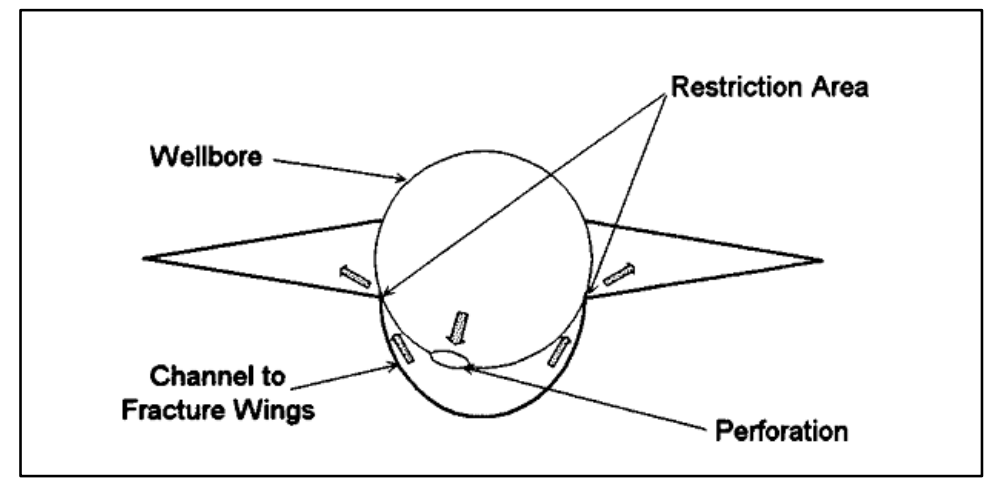

Figure 6.8: Near wellbore effects (Romero, Mack, and Elbel, 1995) 
Microfractures are created in the cement bond during perforation or pumping fracturing fluid. Maintaining a good bond during the breakdown phase is not always achieved. A loose cement bond increases the chance of creating a micro-annulus during formation breakdown. For normal completion practices, the creation of a micro-annulus should be anticipated during the breakdown process. The micro-annulus initiation and propagation is similar to the mechanism that governs the propagation of a hydraulic fracture, but on a smaller scale and confined to the annular circumference of the cement's interface with the well. This phenomena can happen either at the interface of the cement and the casing or the cement and the formation.

A hydraulic fracture, or a micro-annulus, can propagate when fluid of sufficient pressure pressurize a micro-annulus fracture. Microannuli exist in the cement interfaces and around perforation tunnels and are in communication with the wellbore fluid. As the wellbore is pressurized during breakdown, the fluid in the micro-annulus is also pressurized, increasing the width of the micro-annulus by compressing the surrounding material and allowing more fluid to enter and extend the created fracture. The annular width results from the combined radial deformation of the borehole and casing. The evolution of the micro-annulus geometry is similar to that for a hydraulic fracture originating from a point source of injected fluid.

\subsubsection{Bottomhole Treatment Pressure}

To propagate a fracture, energy is required in the form of pressure in the fracturing fluid. Understanding the sources of this pressure gain and loss is important to understand the fracturing process. Types of pressure during hydraulic fracturing operations are listed and explained below. Figure 3.9 shows a schematic of the wellbore and fracture with pressures noted.

\section{Surface Treating Pressure (STP), $P_{\text {surf }}$}

This is also known as wellhead pressure or injection pressure. It is the pressure measured by the gauge at the wellhead where the fracture fluids are pumped through. 


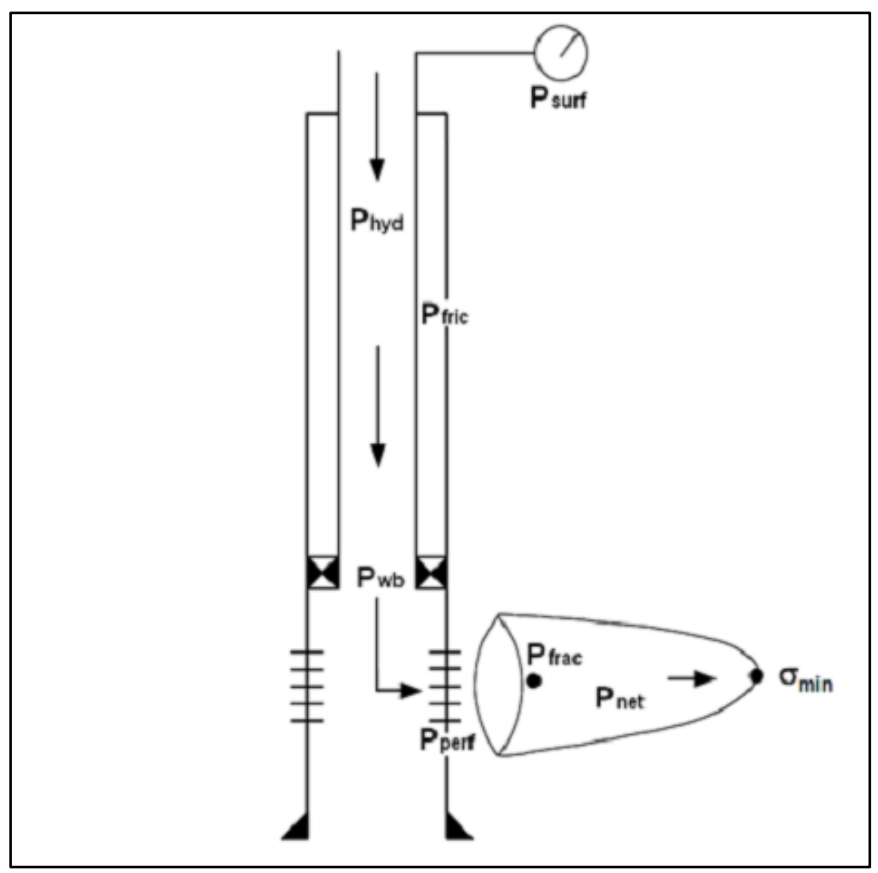

Figure 6.9: Schematic of significant pressures during hydraulic fracturing treatment

\section{Hydrostatic Pressure, $P_{\text {hyd }}$}

$P_{h y d}$ is the hydrostatic pressure exerted by the fracture fluid due to column of fluid in the wellbore and its density. In petroleum engineering fields, it is calculated as:

$P_{\text {hyd }}=0.0052 * \rho * h$

where $\rho$ is the slurry density (lb/gal) and $\mathrm{h}$ is the total vertical depth (ft).

\section{Fluid Friction Pressure, $P_{\text {fric }}$}

This is also referred to as tubing friction pressure or wellbore friction pressure. It is the pressure loss due to friction effect in the wellbore as fluids are injected.

\section{Bottomhole Treating Pressure (BHTP), $P_{w b}$}

BHTP is also referred to as wellbore pressure. It is the downhole pressure, in the wellbore, at the center of the interval being treated. BHTP can be calculated from surface data as follows:

$$
P_{w b}=P_{w b}+P_{h y d}-P_{f r i c}
$$




\section{Perforation Friction Pressure, $\Delta P_{\text {perf }}$}

This is the pressure loss as the fracturing fluid passes through the restricted flow area of the perforations. Perforation friction pressure can be calculated by

$\Delta P_{\text {perf }}=\frac{0.2369 q^{2} \rho}{N_{p}^{2} d_{p}^{4} C_{d}^{2}}$

Where $\rho$ is slurry density, lb/gal, is total flow rate, bbl/min, $N_{p}$ is number of perforations, $d_{p}$ is initial perforation diameter, in., and $C_{d}$ is coefficient of discharge.

\section{Tortuosity Pressure, $P_{\text {tort }}$}

Tortuosity pressure is the pressure loss as fracture fluid passes through a region of restricted flow between the perforation and the main body of the fracture.

\section{Fracturing Fluid Pressure, $P_{\text {frac }}$}

This pressure is the pressure of the fracturing fluid inside the main body of the fracture, after it has passed through the perforations and any tortuous path. Fracturing fluid pressure may not be constant over the entire fracture due to friction effect inside the fracture. It is calculated as follows

$P_{\text {frac }}=P_{w b}-\Delta P_{\text {perf }}$

\section{In-Situ Stress, $\sigma_{h}$}

This is also referred to as closure pressure or minimum horizontal principal stress. It is the stress within the formation, which acts as a load on the formation. It is also the minimum stress required inside the fracture in order to keep it open. For a single layer, it is usually equal to the minimum horizontal stress, allowing for the effect of pore pressure. Otherwise, it is the average stress over all the layers.

\section{Net Pressure, $P_{\text {net }}$}

Net pressure is the excess pressure in the fracturing fluid inside the fracture required to keep the fracture open. Net pressure can be calculated as follows 
$P_{\text {frac }}=P_{w b}-\Delta P_{\text {perf }}$

The importance of the net pressure cannot be overemphasized during fracturing. The net pressure, multiplied by the fracture volume, provides us with the total quantity of energy available at any given time to make the fracture grow. How that energy is used during generation of width, splitting of rock, fluid loss or friction loss is determined by the fracture model being employed to simulate fracture growth.

\subsection{Experimental Investigation of Fracture Propagation from Perforation}

Several studies in the literature have investigated the effect of perforations on hydraulic fracture initiation and propagation. The first work on the effect of perforation on hydraulic fracturing was presented by Daneshy (Daneshy, 1973) who showed that the direction of induced hydraulic fracture is not dictated by perforation orientation. His work showed that in many cases, fluid can travel from the perforation through the area between the casing and formation to initiate a fracture in the direction of maximum horizontal stress.

In an experimental work, El Rabaa (El-rabaa, 1982) examined effect of different parameters in perforation design and showed that multiple fractures could be created when the perforated interval was longer than four times well-bore diameters. Soliman (Soliman, 1990) showed that fracture reorientation may affect the analysis of microfrac and minifrac tests in horizontal wells. Hallam et al. in their study (Hallam and Last, 1991) recommended that without knowledge of the stress directions, a low phasing angle should be used. Kim and Abass (Kim and Abass, 1991) showed that for wells with high deviation angles, a pair of mutually perpendicular fractures was created and was injection-rate dependent.

In another series of experiment (Abass, Hedayati, and Meadows, 1996; Abass et al., 1994) using laboratory experimental examined the effect of oriented perforation in vertical and horizontal wells on hydraulic fracturing treatment and sand control. Results of their experiment showed the relation between the breakdown pressure and hydraulic fracture width and perforation orientation. 
There are several other experimental studies on hydraulic fracturing initiation and propagation conducted on rock and sediment samples (Hanson et al., 1982), cement paste (de Pater et al., 1994), and gypsum cement (Abass, Hedayati, and Meadows, 1996). A common difficulty with these tests is the observation and measurement of the hydraulic fractures that develop inside these materials. Generally, the induced fracture geometry is observed by cutting the sample after the test or by using an acoustic monitoring system. By cutting the samples after the test, only the final results are observed and also there is the risk to alter the created fracture during cutting process.

There are also laboratory experiments on hydraulic fracturing in transparent materials that allowed the visualization in real time of the developing geometry of the fracture (Bunger, Jeffrey, and Detournay, 2004) and the direction of fracture propagation (Hubbert and Willis, 1957; Bakala, 1997). Commonly used transparent geo-material analogues for fracturing are polymethylmethacrylate (PMMA, acrylic), polycarbonate, silica glass, polyester resin, gelatin, and acrylic.

Control of fracture orientation is important for the interpretation of laboratory results. To have some control over the fracture direction, usually these samples are perforated. Several methods have been utilized to improve the control of the fracture orientation. For example, a starter fracture was sometimes implemented to reduce the fracture initiation pressure. Bunger et al. (2004) created a starter fracture by inserting a rod into the injection tube and striking it firmly with a hammer. Germanovich et al. (1999) created an initial notch by rotating a bent, sharpened rod inside the drill hole to scratch out PMMA material. 


\section{Chapter 4}

\section{Modeling Fracture Propagation from Perforations}

This chapter introduces model specification, parameters and assumptions and the approach to build a finite element models in the commercial FE simulator Abaqus to study fracture propagation from oriented perforations. The theories to justify the method are discussed, some challenges related to applying XFEM in the model are addressed and the approaches taken to overcome these challenges are presented. Most of these challenges are due to the fact that XFEM formulation is new in Abaqus and some features have not yet been reformulated to adapt this capability.

We start by going through model specifications and similarities with experimental work performed in the literature. Then model parameters, assumptions and boundary conditions are discussed. The chapter is completed with the important steps to use XFEM analysis in Abaqus with brief description of theory and keyword used.

\subsection{Model Description}

The model to study the effect of perforation orientation is based on some experimental studies (Abass et al., 1994; Abass, Hedayati, and Meadows, 1996). Their laboratory experiments were designed to investigate the effect of perforation orientation in vertical and horizontal wells on hydraulic fracturing treatment. Dimensions and parameters in our study are selected to be similar to the ones in those experiments in order to cross check some of the results.

Rock samples used in these experiments were rectangular blocks of hydrostone (gypsum cement) with dimensions of $6 \times 6 \times 10$ inches. These blocks were created from mixing water and hydrostone with a weight ratio of 32/100, respectively. A wellbore was drilled in the center of the block in the direction of the 10-in. side. The wellbore was cased and perforated. A series of perforation orientations was considered: $\theta=0^{\circ}, 15^{\circ}$, $30^{\circ}, 45^{\circ}, 60^{\circ}, 75^{\circ}$, and $90^{\circ}$ from the Preferred Fracture Plane(PFP). All samples were confined in a triaxial loading vessel and the principal stresses of 3000 psi vertical, 2500 
psi maximum horizontal, and 1400 psi minimum horizontal stresses were applied. No pore fluid was present within the sample blocks (Abass, Hedayati, and Meadows, 1996).

A schematic of core sample is illustrated in figure 4.1. Also, the physical and mechanical properties are listed in table 1.

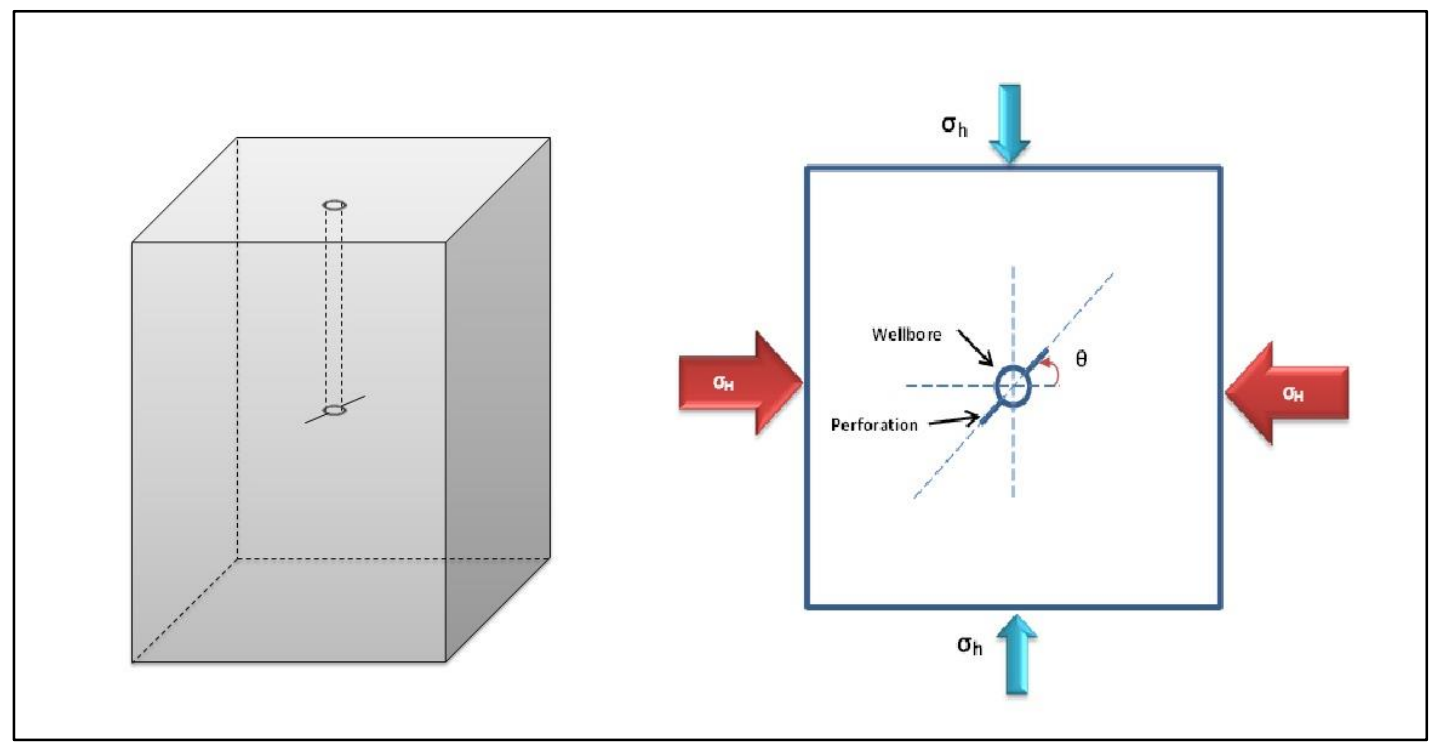

Figure 4.1: Schematic of simulation model

Table 4.1: Physical and mechanical properties of the sample used in the model

\begin{tabular}{|l|l|l|}
\hline Rock Sample Properties & Values & SI Unit \\
\hline Dimension & $6 \times 6 \times 10 \mathrm{in}$ & $0.1524 \times 0.1524 \times 0.254 \mathrm{~m}$ \\
\hline Well radius & $0.25 \mathrm{in}$ & $0.00635 \mathrm{~m}$ \\
\hline Perforation length & $0.25 \mathrm{in}$ & $0.00635 \mathrm{~m}$ \\
\hline Young Modulus & $2.07 \mathrm{e}+06 \mathrm{psi}$ & $1.427 \mathrm{e}+10 \mathrm{~Pa}$ \\
\hline Poisson ratio & 0.21 & 0.21 \\
\hline Min Horizontal Stress & $1400 \mathrm{psi}$ & $9.65 \mathrm{e}+6 \mathrm{~Pa}$ \\
\hline Max Horizontal Stress & $2500 \mathrm{psi}$ & $17.24 \mathrm{e}+6 \mathrm{~Pa}$ \\
\hline Applied vertical Stress & $3000 \mathrm{psi}$ & $20.68 \mathrm{e}+6 \mathrm{~Pa}$ \\
\hline Sample Permeability & $39 \mathrm{mD}$ & $0.039 \mathrm{D}$ \\
\hline Porosity & $26.5 \%$ & 0.265 \\
\hline Uniaxial compressive strength & $8032 \mathrm{psi}$ & $5.538 \mathrm{e}+7 \mathrm{~Pa}$ \\
\hline Tensile strength (Brazilian) & $807.6 \mathrm{psi}$ & $5.568 \mathrm{e}+6 \mathrm{~Pa}$ \\
\hline
\end{tabular}


A consistent system of units must be used in Abaqus; therefore all magnitudes are converted to SI units which can be seen in the last column of Table 4.1.

Since the samples used for those experiments are cut to observe the fracture propagation pattern, it is a good idea to consider only a 2D model as shown in figure 4.1 (right). The 2D model used can save simulation time and the results can be compared to available photographs taken from the sample after being cut.

\subsection{Node, Element, Mesh}

As discussed in chapter 2, XFEM was first introduced in 1999 and during the last 15 years, there has been some attempts to implement the method in finite element commercial software. XFEM module appeared in Abaqus for the first time in 2009 with the Abaqus 6.9 release (Dassault Systemes, 2009). Since then and with each release of the software, more features are added to include XFEM in different capabilities of Abaqus. There are still some limitations when applying this method in Abaqus. The limitations are in some features including element types, analysis methods and types of load and boundary condition. Some of these features not compatible with XFEM in Abaqus which affect this study are

- Pore-Pressure element types

- Geostatic step

- Initial pore pressure

- Quadratic elements

Some methods were devised to compensate for these limitations and are discussed in the corresponding sections.

In XFEM, fracture is modeled independent of mesh configuration and element type. This means that no remeshing is required and discontinuity including fractures need not to be aligned with element boundaries. However, type of element and mesh configuration have some effects on simulation convergence and results. Therefore, different available element types was examined to come up with the optimum mesh configuration which has reasonable simulation run time without compromising the results and data resolution. 
Based on the discussion above and after examining different element types and mesh configuration, the Structured, Standard, Linear Quadrilateral Plane Strain Element type (CPE4) has been selected for this model. Most of the models throughout this study have 1526 Nodes, 1440 elements or in some cases 23368 nodes, 23044 elements where finer mesh configuration is required.
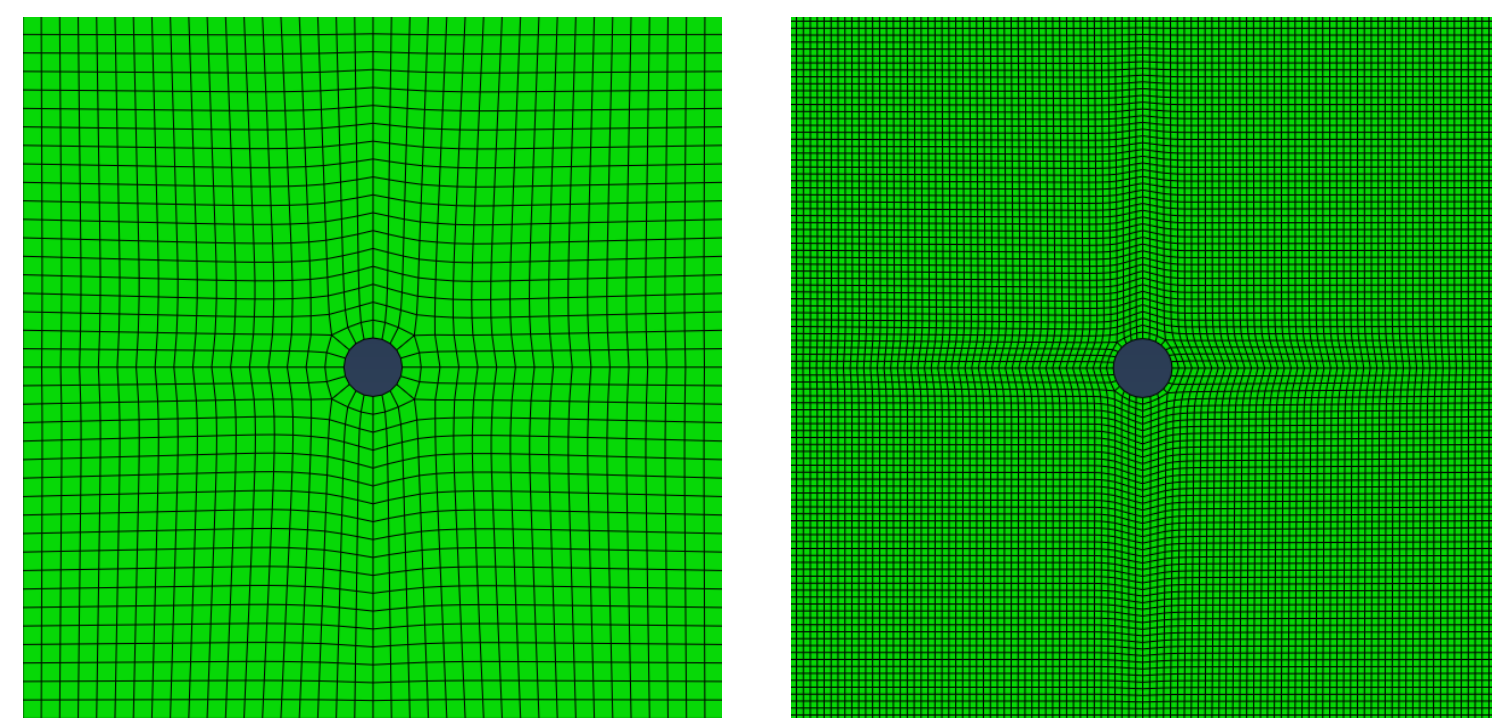

Figure 4.2: Two mesh configurations used for the analyses throughout the study

The finer mesh is specially suitable for smaller initial perforation type. The XFEM method in Abaqus, forces the initial discontinuity to cut the whole element immediately. Therefore, if initial perforations extends halfway the element length, it will automatically be extended to the element boundary which results in a different initial fracture length. Finer element size, will minimize this effect.

Pore pressure element type is not compatible with XFEM analysis in Abaqus, therefore Plane Strain elment types are used. Plane strain elements can be used where the loading and stress are not changing in one direction (usually z-direction) and the loads are applied in the plane of the element (x-y plane) and are not varying in z-direction. In plane strain elements, thickness is equal to unity and the strain components perpendicular to the element face are zero: $\varepsilon_{z z}=0$. Some important application of this representation occur in the analysis of geotechnical structures including hydraulic fracturing in rock formation, 
where the strain in the z-dirction (dirction of increasing depth) is neglegible compared to strain in the other two directions.

\subsection{Stress Initialization}

Abaqus uses the initial stresses specified by the user as an initial guess or as a starting point in the process of getting an initial stresses for the start of the analysis. This initial stress has a great effect on the subsequent steps. Deviation of the initial stress values from the actual one, would result in incorrect displacements of the model which in turn results in instabilities and analysis termination.

Abaqus calculates the stresses which are in equilibrium with the external loading and boundary conditions. A geostatic step is defined after initial step to reach a stress equilibrium. The displacement that occur during the geostatic step is not because of the external loading but due to the difference between the user predicted initial stresses and the stresses calculated by Abaqus.

Abaqus checks for equilibrium during the geostatic procedure and iterates, if needed, to obtain a stress state that equilibrates the applied boundary conditions and loads. This stress state, which is then used as the initial stress field in a subsequent static analysis.(Dassault Systèmes, 2013)

As a current limitation of Abaqus, XFEM analysis cannot be combined with geostatic step or pore pressure elements. Since the laboratory experiments described at the beginning of this chapter, are not including pore pressure effect, ignoring pore pressure elements is not far from experiment conditions. However, the attempt is to have the element as similar to porous media by including permeability, porosity and their initial values in the material property. For incompatibility with Geostatic step, the problem was compensated by creating the base model without XFEM analysis, running the model without initial perforation and using corresponding database file (.odb) as the restart point for XFEM analysis. The Base model must have the same properties and meshing configuration as the main model and must be run in the same folder. 
Figure 4.3 shows the initialized in-situ horizontal stress distribution as the output of the base model. As shown in this figure the initial horizontal stress has been redistributed around wellbore.

Initial stress in the rock usually has a homogeneous distribution and is a function of local tectonic activities. When a borehole is drilled in a formation the rock materials are removed and this will undergo a change in homogenous distribution of initial horizontal stresses. There are several models in the literature for simulating the wellbore stress profile. The equations describing the concentration of stresses in a plate with a circular hole under uniform tension at infinity were first derived by G.Kirsch in 1898 . One of the early works contributing to the knowledge of stress distribution around a borehole was published by Westergaard (Westergaard, 1940), in which an elasto-plastic model was developed. Some others have tried to develop a linear elastic constitutive model (Bradley, 1979). Among numerous published models, linear elastic approach is the most common model, because it is easy to use and requires fewer inputs.
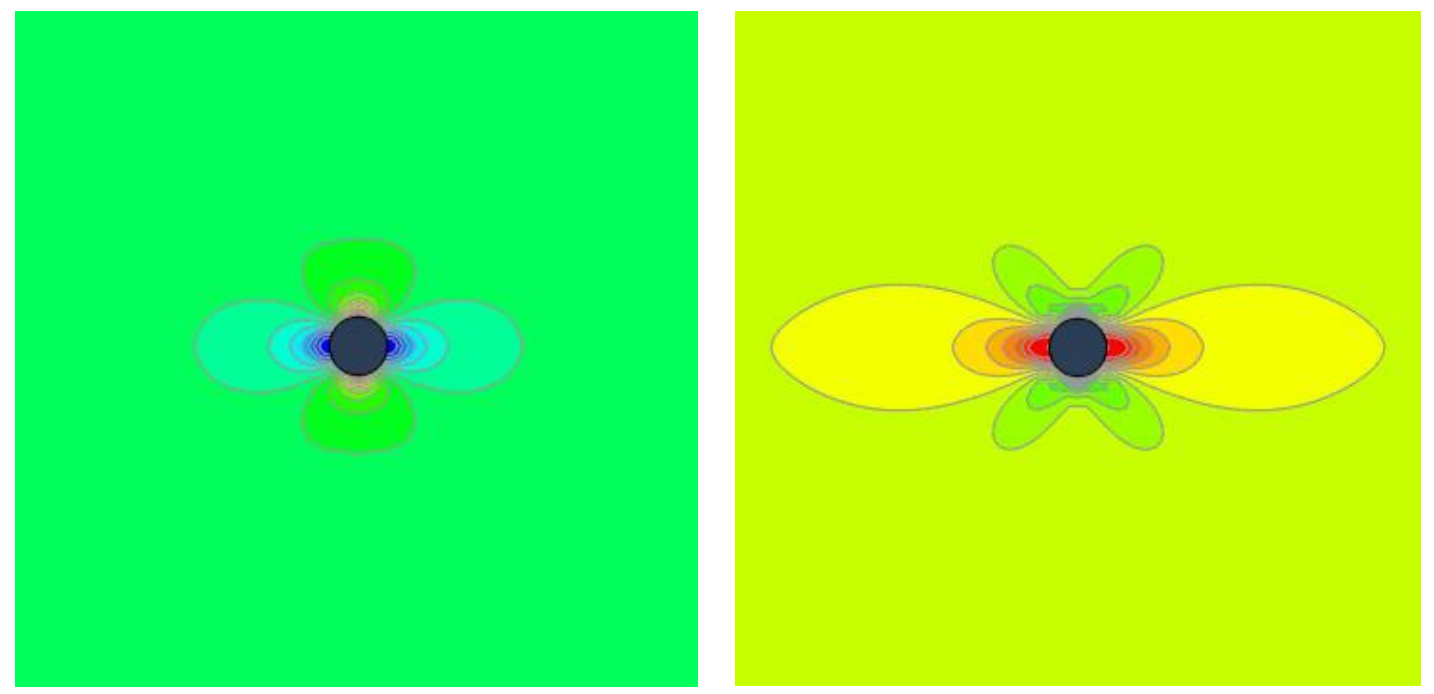

Figure 4.3: Initialization of horizontal in-situ stresses S11 (left) S22 (right)

It is usually a good idea to express the stresses in cylindrical coordinates $r, \theta$ and $\mathrm{z}$, where $\mathrm{r}$ is the distance from the wellbore, $\theta$ the azimuth angle from the $\mathrm{x}$-axis, and $\mathrm{z}$ is in the direction of wellbore axis. Ignoring the pore pressure and shear stress on $\mathrm{x}-\mathrm{y}$ plane, the cylindrical normal stresses can be written as: 


$$
\begin{aligned}
& \sigma_{r}=\frac{\sigma_{H}+\sigma_{h}}{2}\left(1-\frac{r_{w}^{2}}{r^{2}}\right)-\frac{\sigma_{H}-\sigma_{h}}{2}\left(1+3 \frac{r_{w}^{4}}{r^{4}}-4 \frac{r_{w}^{2}}{r^{2}}\right) \cos (2 \theta) \\
& \sigma_{\theta}=\frac{\sigma_{H}+\sigma_{h}}{2}\left(1+\frac{r_{w}^{2}}{r^{2}}\right)-\frac{\sigma_{H}-\sigma_{h}}{2}\left(1+3 \frac{r_{w}^{4}}{r^{4}}\right) \cos (2 \theta)
\end{aligned}
$$

In these equations $\sigma_{\theta}$ is the tangential stress around the wellbore and $\sigma_{\mathrm{r}}$ is the radial stress, $r_{w}$ is the wellbore radius while $r$ is distance in radial direction. Angle $\theta$ is measured from the maximum horizontal stress in positive $\mathrm{x}$ direction. The results for tangential and radial stress distribution around a wellbore in our model are plotted in figure 4.4. It should be noted that these plots are in radial coordinate and cannot be compared to results in figure 4.3.
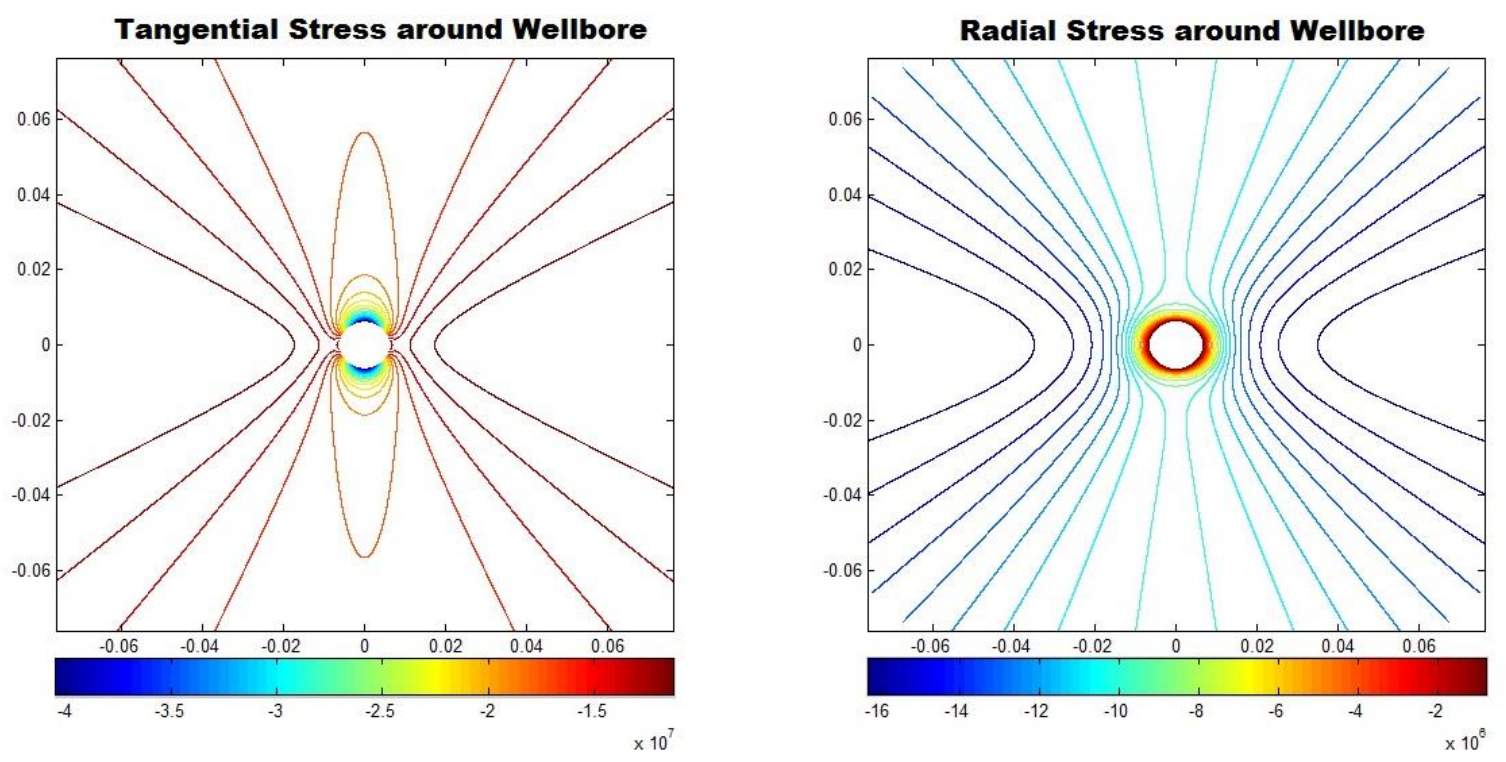

Figure 4.4: Tangential and radial stress distribution around wellbore in radial coordinate 


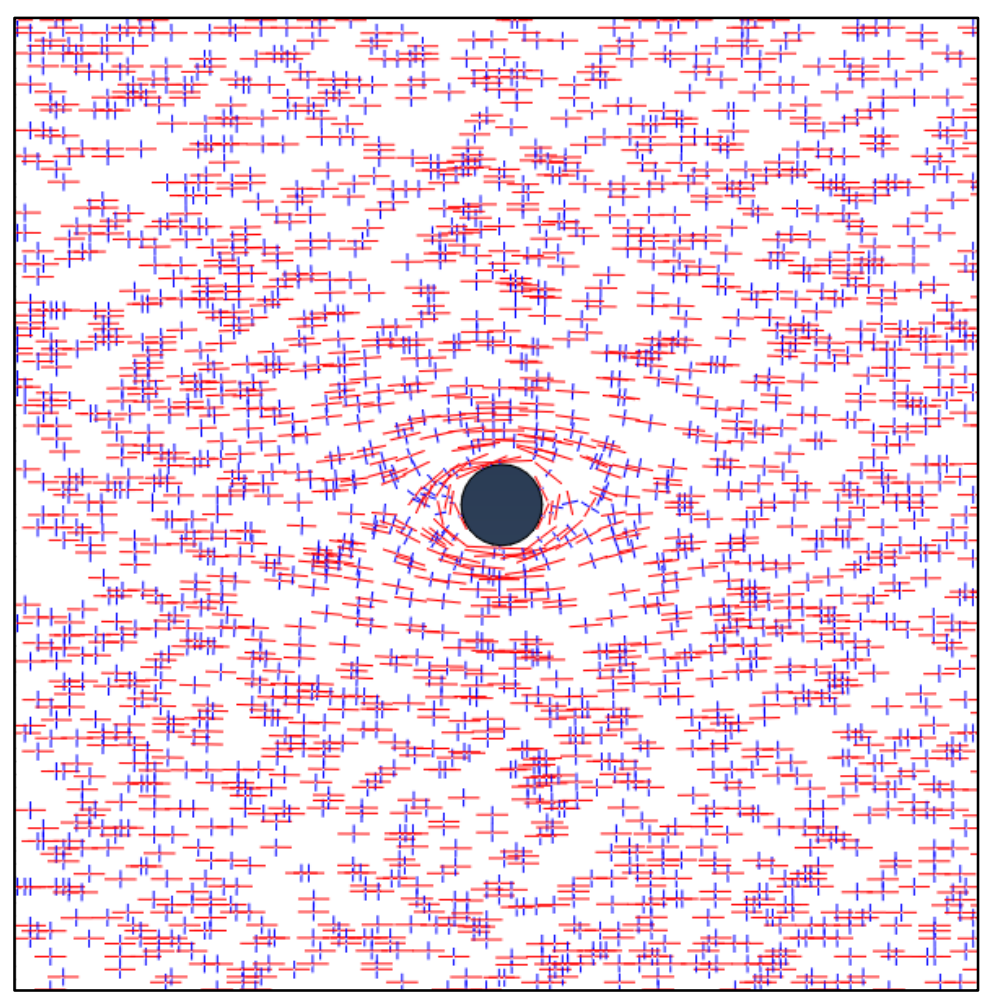

Figure 4.5: Tensor representation of principal horizontal stresses

Figure 4.5 shows the tensor representation of horizontal principal stresses in the model after initial stresses are applied. In this figure Max principal stress are in red and min principal stress are in blue.

\subsection{Boundary Conditions}

Boundary conditions in Abaqus can be applied to displacement DOFs. The boundary conditions should be in equilibrium with the initial stresses and applied loads. Since in the experimental model, the core sample is bounded and fixed in one direction, Displacement/Rotation type of boundary condition are applied to model outer boundaries as shown in figure 4.6.

As the right illustration in figure 4.6 shows there is a gap between the two adjacent metal slabs at the corner, therefore the experimental set-up cannot apply a perfect no-displacement boundary on the rock sample. 

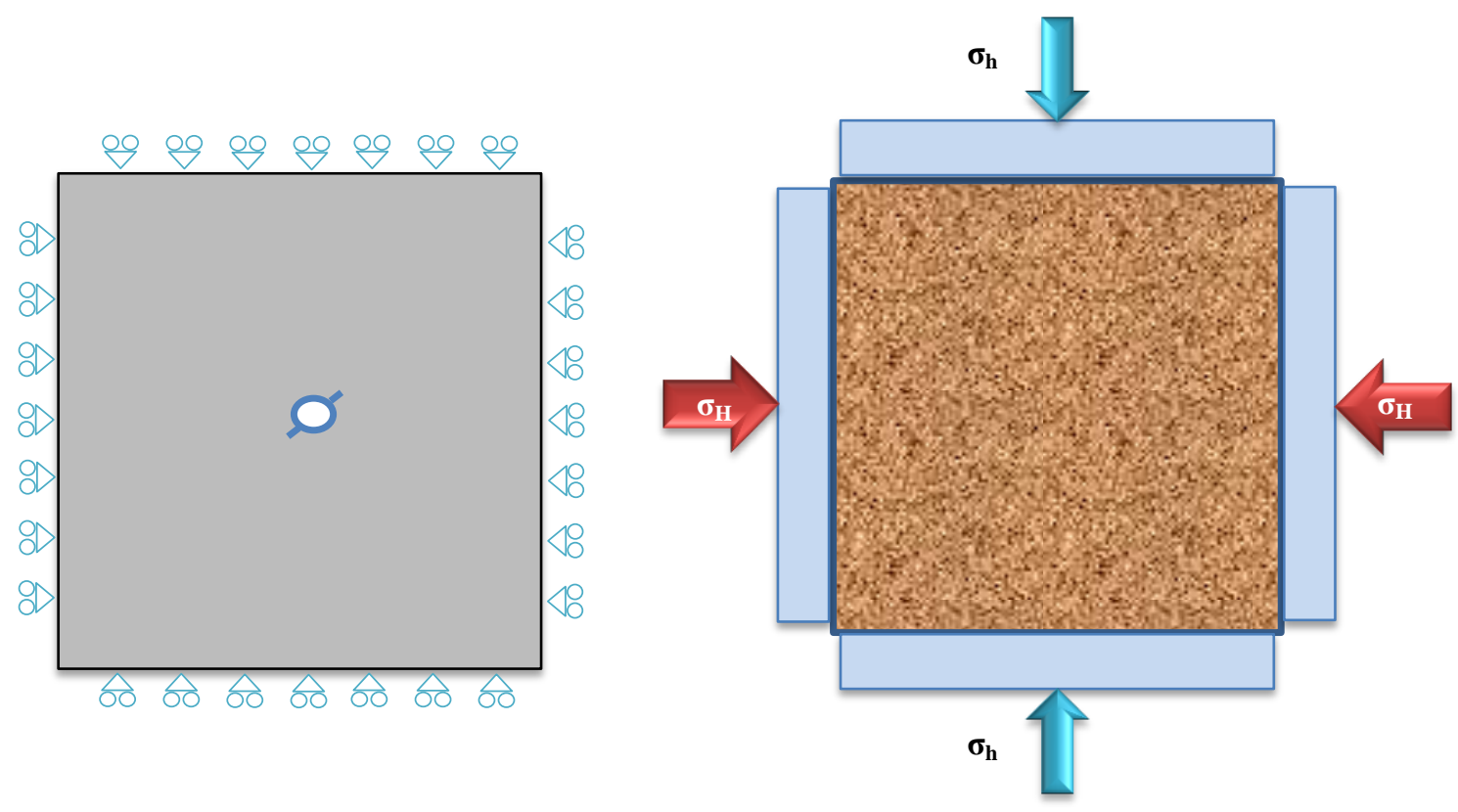

Figure 4.6: Displacement boundary condition applied to the model

\subsection{Perforations}

Perforations are created to serve as communication channels between wellbore and formation (core) and to decrease breakdown pressure and ease fracture propagation. There are several methods to create a perforation in a well drilled and cased in the laboratory. For example Bunger et al. (2004) created a starter fracture by inserting a rod into the injection tube and striking it firmly with a hammer. Germanovich et al. (1999) created an initial notch by rotating a bent, sharpened rod inside the drill hole to scratch out the material. Unfortunately, no information is provided on how the wells were perforated in the experiment targeted in the study by Abass et al.

In the model used throughout this study, perforations are modeled as initial fractures extended from wellbore in opposite direction $\left(180^{\circ}\right.$ phasing). Two wings are considered to have the same length and zero initial width and positioned at angle $\theta$ measured from maximum horizontal stress and increasing counter clock wise. A sensitivity analysis has been done on perforation orientation angle and initial length which will be presented in chapter 5. Schematic of wellbore, initial perforation and parameters involved are presented in figure 4.7. 


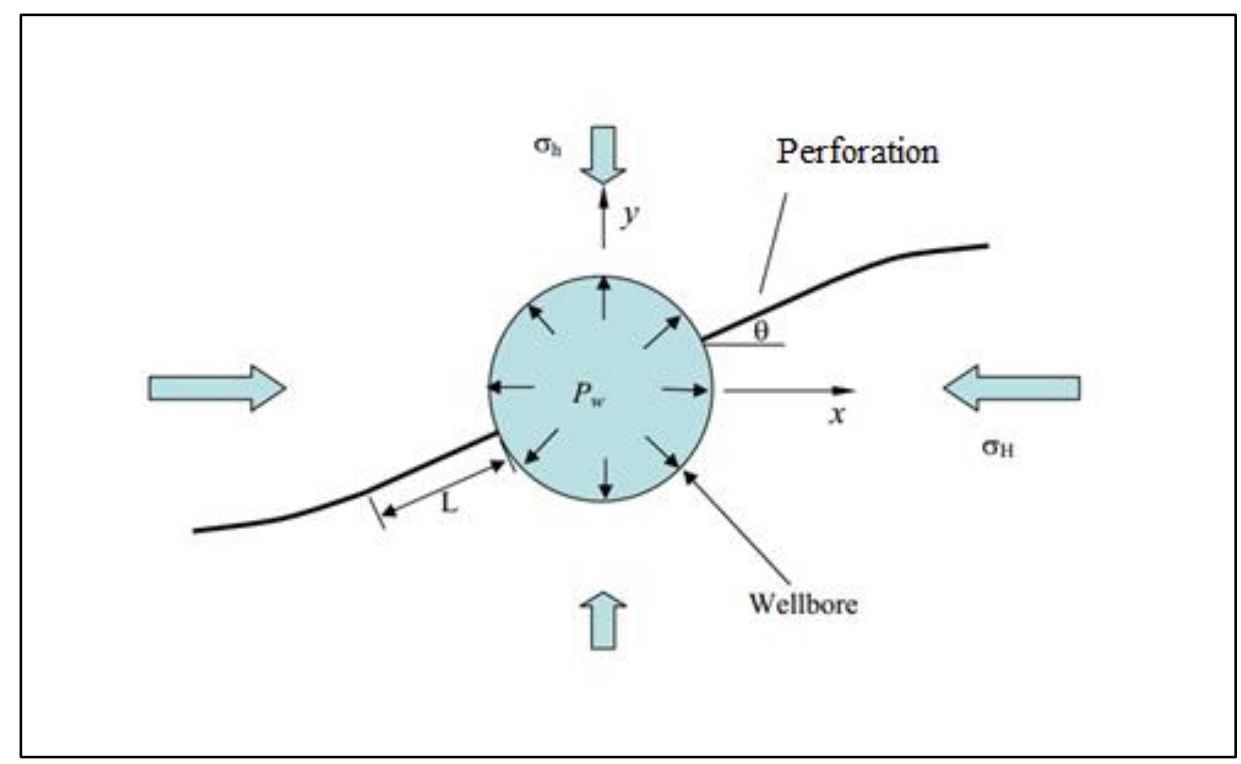

Figure 4.7: Wellbore, initial perforation and parameters involved

No casing or cement is considered in this model; therefore fractures are initiated and propagated from the perforation tip. Depending on the perforation length and orientation, it will take a distance of few wellbore radiuses for the propagating fracture to exit the near wellbore region and reorient in the direction PFP. The results will be discussed in next chapter.

\subsection{Defining Enriched Feature and its Properties in Abaqus}

To use XFEM in Abaqus, the enriched feature and its properties must be specified. One or multiple fracture can be defined for an enriched feature. In addition, during an analysis one or more fracture can initiate in an enriched feature when the fracture initiation criterion is reached. Enrichments are activated only when an element is cut by a fracture. (Dassault Systèmes, 2013). Table 4.2 summarizes the keywords and their properties in Abaqus input file.

Some of these features can also be applied in GUI as well, while others have to be strictly added manually to the input file. Input file gives more flexibility and is easier to modify especially when running several simulations with few small changes. 
Table 4.2: Summary of some of keywords used to define enrichment in Abaqus (Dassault Systemes, 2009)

\begin{tabular}{|c|c|c|}
\hline Category & Keyword & Comments \\
\hline \multirow{2}{*}{ type of enrichment } & $\begin{array}{l}\text { *ENRICHMENT, } \\
\text { TYPE=PROPAGATION CRACK }\end{array}$ & $\begin{array}{l}\text { The cohesive segments method and linear } \\
\text { elastic fracture mechanics approach in } \\
\text { conjunction with phantom nodes. }\end{array}$ \\
\hline & $\begin{array}{l}\text { *ENRICHMENT, } \\
\text { TYPE=STATIONARY CRACK }\end{array}$ & $\begin{array}{l}\text { Analysis with stationary cracks requires } \\
\text { that the elements around the crack tips are } \\
\text { enriched with asymptotic functions }\end{array}$ \\
\hline Enriched region & $\begin{array}{l}\text { *ENRICHMENT, ELSET=element } \\
\text { set name }\end{array}$ & $\begin{array}{l}\text { Elements currently intersected by cracks } \\
\text { and those likely to be intersected by cracks }\end{array}$ \\
\hline Crack surface & *SURFACE, TYPE=XFEM & $\begin{array}{l}\text { Representing both faces of cracked } \\
\text { elements }\end{array}$ \\
\hline $\begin{array}{l}\text { Cracked element } \\
\text { surfaces }\end{array}$ & $\begin{array}{l}\text { *ENRICHMENT, INTERACTION } \\
=\text { interaction_property_name }\end{array}$ & $\begin{array}{l}\text { Feature such as cohesive behavior can be } \\
\text { added as interaction }\end{array}$ \\
\hline Crack initiation & $\begin{array}{l}* \text { DAMAGE INITIATION, } \\
\text { TOLERANCE }=f_{\text {tol }}\end{array}$ & The default value of $f_{t o l}$ is 0.05 \\
\hline \multirow{3}{*}{$\begin{array}{l}\text { Position used to } \\
\text { measure the crack } \\
\text { initiation criterion }\end{array}$} & $\begin{array}{l}\text { *DAMAGE INITIATION, } \\
\text { POSITION=CENTROID (default) }\end{array}$ & \multirow{3}{*}{$\begin{array}{l}\text { By default, Abaqus uses a Gauss point } \\
\text { average of the stress/strain evaluated at the } \\
\text { element centroid ahead of the crack tip } \\
\text { Alternatively, you can use the stress/strain } \\
\text { values extrapolated to the crack tip }\end{array}$} \\
\hline & $\begin{array}{l}\text { *DAMAGE INITIATION, } \\
\text { POSITION=CRACKTIP }\end{array}$ & \\
\hline & $\begin{array}{l}\text { *DAMAGE INITIATION, } \\
\text { POSITION=COMBINED }\end{array}$ & \\
\hline $\begin{array}{l}\text { Specifying the crack } \\
\text { direction }\end{array}$ & $\begin{array}{l}\text { *DAMAGE INITIATION, } \\
\text { NORMAL DIRECTION =1 (default) } \\
\text { *DAMAGE INITIATION, } \\
\text { NORMAL DIRECTION }=2\end{array}$ & $\begin{array}{l}\text { Newly introduced crack is always } \\
\text { orthogonal to the maximum principal } \\
\text { stress/strain direction } \\
\text { you can specify if the newly introduced } \\
\text { crack will be orthogonal to the element } \\
\text { local 1-direction }\end{array}$ \\
\hline $\begin{array}{l}\text { Activating and } \\
\text { deactivating the } \\
\text { enriched feature }\end{array}$ & $\begin{array}{l}\text { *ENRICHMENT ACTIVATION, } \\
\text { NAME=name, ACTIVATE=ON } \\
\text { *ENRICHMENT ACTIVATION, } \\
\text { NAME=name, ACTIVATE=OFF }\end{array}$ & $\begin{array}{l}\text { By default, enrichment is activated and can } \\
\text { be deactivated/reactivated in a new step }\end{array}$ \\
\hline
\end{tabular}


The enriched element can have all the properties of any other elements available for XFEM, except the following limitations exist with an enriched feature:

- An element cannot be cut by more than one fracture.

- A fracture is not allowed to turn more than $90^{\circ}$ in one increment during an analysis.

- Only asymptotic fracture-tip fields in an isotropic elastic material are considered for a stationary fracture

- Adaptive remeshing is not supported.

Most of these limitations are due to the XFEM being a new formulation and recently added to Abaqus and are expected to be removed in future versions (Dassault Systemes, 2009).

\subsection{Fracture Initiation and Extension in Abaqus}

Fracture initiation occurs when cohesive response at an enriched element starts to degrade. This will happen when the stresses or the strains satisfy specified fracture initiation criteria. Fracture initiation criteria available in Abaqus are based on built-in models which are summarized in table 4.3. In addition, a user-defined fracture initiation criterion can be used in subroutine UDMGINI. (Dassault Systèmes, 2013)

A fracture is initiated or the length of an existing fracture is extended after equilibrium increment when the fracture criterion, $f$, reaches the value 1.0 within a specified tolerance:

$1.0 \leq f \leq 1.0+f_{\text {tol }}$

The tolerance $f_{\text {tol }}$ can be given by user. The default value of $f_{\text {tol }}$ is 0.05 . If $f>$ $1.0+f_{\text {tol }}$, the time increment is cut back such that the fracture initiation criterion is satisfied. 
Table 4.3: Damage initiation criterion and their implementation in abaqus

\begin{tabular}{|c|c|c|}
\hline Criterion & $f$ & Keyword \\
\hline Maximum principal stress & $f=\left\{\frac{\left\langle\sigma_{\max }\right\rangle}{\sigma_{\max }^{o}}\right\}$ & $\begin{array}{l}\text { *DAMAGE INITIATION, } \\
\text { CRITERION=MAXPS }\end{array}$ \\
\hline Maximum principal strain & $f=\left\{\frac{\left\langle\varepsilon_{\max }\right\rangle}{\varepsilon_{\max }^{o}}\right\}$ & $\begin{array}{l}\text { *DAMAGE INITIATION, } \\
\text { CRITERION=MAXPE }\end{array}$ \\
\hline Maximum nominal stress & $\begin{array}{l}f=\max \left\{\frac{\left\langle t_{n}\right\rangle}{t_{n}^{0}}, \frac{t_{s}}{t_{s}^{0}}, \frac{t_{t}}{t_{t}^{0}}\right\} \\
\mathrm{t}_{\mathrm{n}} \text { is normal and } \mathrm{t}_{\mathrm{s}} \text { and } \mathrm{t}_{\mathrm{t}} \text { are shear } \\
\text { components } \\
t_{n}^{0}, t_{s}^{0}, t_{t}^{0} \text { represent the peak values of } \\
\text { the nominal stress }\end{array}$ & $\begin{array}{l}\text { *DAMAGE INITIATION, } \\
\text { CRITERION=MAXS }\end{array}$ \\
\hline Maximum nominal strain & $f=\max \left\{\frac{\left\langle\varepsilon_{n}\right\rangle}{\varepsilon_{n}^{0}}, \frac{\varepsilon_{s}}{\varepsilon_{s}^{0}}, \frac{\varepsilon_{t}}{\varepsilon_{t}^{0}}\right\}$ & $\begin{array}{l}\text { *DAMAGE INITIATION, } \\
\text { CRITERION=MAXE }\end{array}$ \\
\hline Quadratic nominal stress & $f=\left\{\frac{\left\langle t_{n}\right\rangle}{t_{n}^{0}}\right\}^{2}+\left\{\frac{t_{s}}{t_{s}^{0}}\right\}^{2}+\left\{\frac{t_{t}}{t_{t}^{0}}\right\}^{2}$ & $\begin{array}{l}\text { *DAMAGE INITIATION, } \\
\text { CRITERION=QUADS }\end{array}$ \\
\hline Quadratic nominal strain & $f=\left\{\frac{\left\langle\varepsilon_{n}\right\rangle}{\varepsilon_{n}^{0}}\right\}^{2}+\left\{\frac{\varepsilon_{s}}{\varepsilon_{s}^{0}}\right\}^{2}+\left\{\frac{\varepsilon_{t}}{\varepsilon_{t}^{0}}\right\}^{2}$ & $\begin{array}{l}\text { *DAMAGE INITIATION, } \\
\text { CRITERION=QUADE }\end{array}$ \\
\hline
\end{tabular}

The symbol \langle\rangle is the Macaulay bracket and used to show that a compressive stress state does not initiate damage by itself and the critical value should be achieved before fracture start to grow: $\left(\left\langle\sigma_{\max }\right\rangle=0\right.$ if $\sigma_{\max }<0$ and $\left\langle\sigma_{\max }\right\rangle=\sigma_{\max }$ if $\left.\sigma_{\max } \geq 0\right)$. The fracture initiation is governed by one of the six built-in stress- or strain-based fracture initiation criteria (summarized in table 4.3) or a user-defined fracture initiation criterion. After a fracture is initiated in an enriched region, subsequent propagation of the fracture is governed by the XFEM-based LEFM criterion (Dassault Systemes, 2009). 


\section{Chapter 5}

\section{Results and Discussions}

A XFEM model has been developed in Abaqus to investigate the effect of oriented perforation on fracture initiation and propagation. The result from this model will help to understand the effect of different design parameters involved in hydraulic fracturing from cased holes. Since the experimental investigation of this process is expensive, time consuming and with limited results, the numerical model verified with lab experiments results, can be an efficient way to investigate fracture initiation and propagation from oriented perforation.

After the model described in the previous chapter was built using FE software Abaqus, different sensitivity analyses were performed to get a better understanding of hydraulic fracture propagation from perforations and examine different parameters and design configuration without the need to go through experimental design. In this chapter the effects of perforation angle, perforation length, rock mechanical properties, stress anisotropy and nearby discontinuities on fracture propagation from cased hole are investigated and analyzed.

\subsection{Perforation Angle}

Generally, fractures tend to initiate when the applied pressure overcomes the tensile stress at wellbore and propagate in the direction of PFP. When the wellbore is cased and perforated, perforation will help the initiation of fracture by serving as initial fractures, but the created fracture will not necessarily follow the orientation provided by the perforation. Once passed near wellbore region, the initiated fracture will follow the path of least resistance which is perpendicular to direction of minimum horizontal stress. To investigate this effect, models with perforation at different angles are built and run. The orientation angle is measured from the direction of PFP (which is parallel to the direction of maximum horizontal stress) in positive $\mathrm{x}$ direction. In all cases, perforations 
are of the same length, equal to one wellbore radius, and other properties are kept the same. The results are presented in figures 5.1 to 5.5.

The result from orientation angles 45, 60 and 75 and 90 degrees are compared to some photographs from experimental studies available.
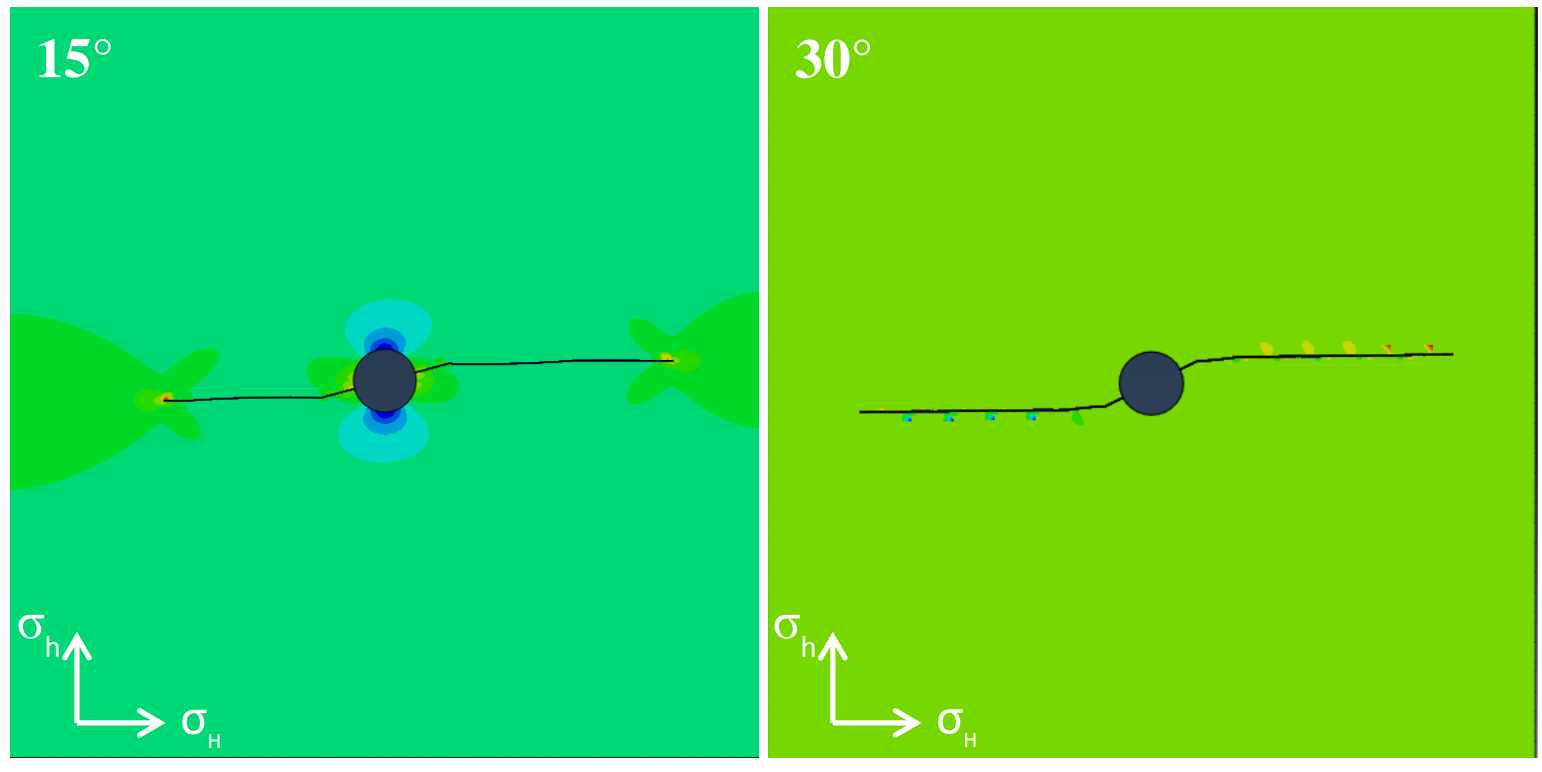

Figure 5.1: Fracture reorientation at $\theta=15^{\circ}$ (left) and $\theta=30^{\circ}$ (right)
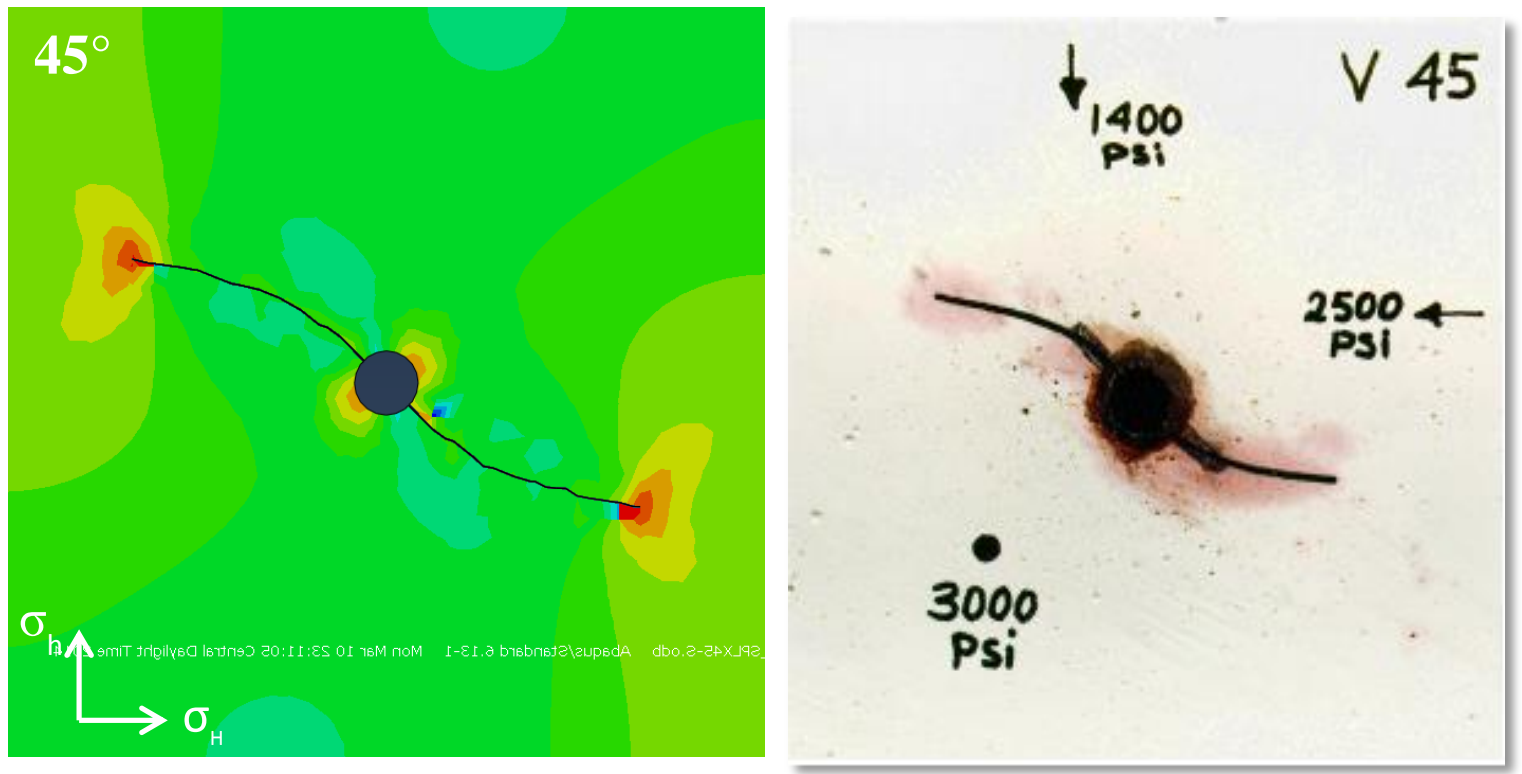

Figure 5.2: Fracture reorientation at $\theta=45^{\circ}$ and comparison to the experimental model 

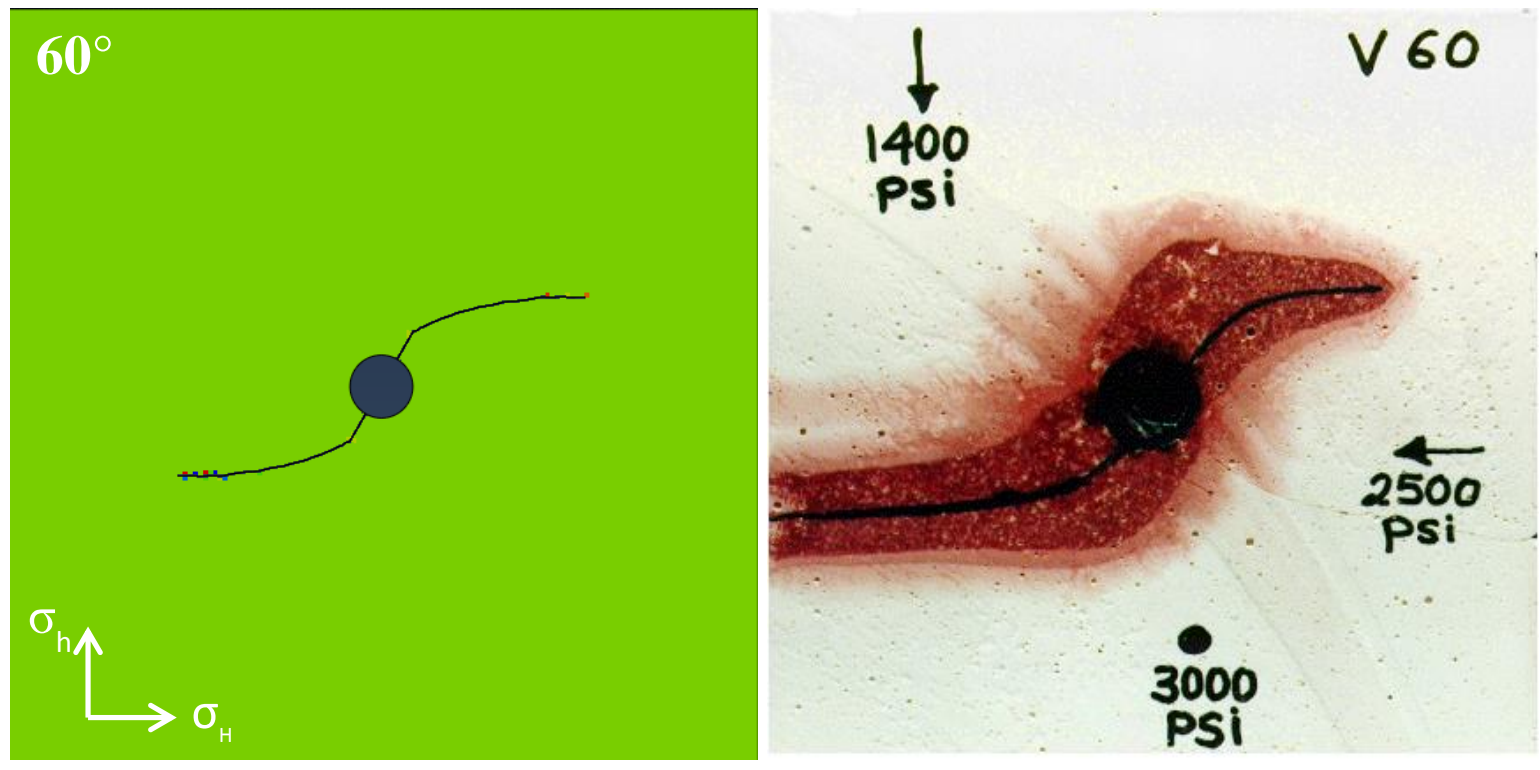

Figure 5.3: Fracture reorientation at $\theta=60^{\circ}$ and comparison to experimental model
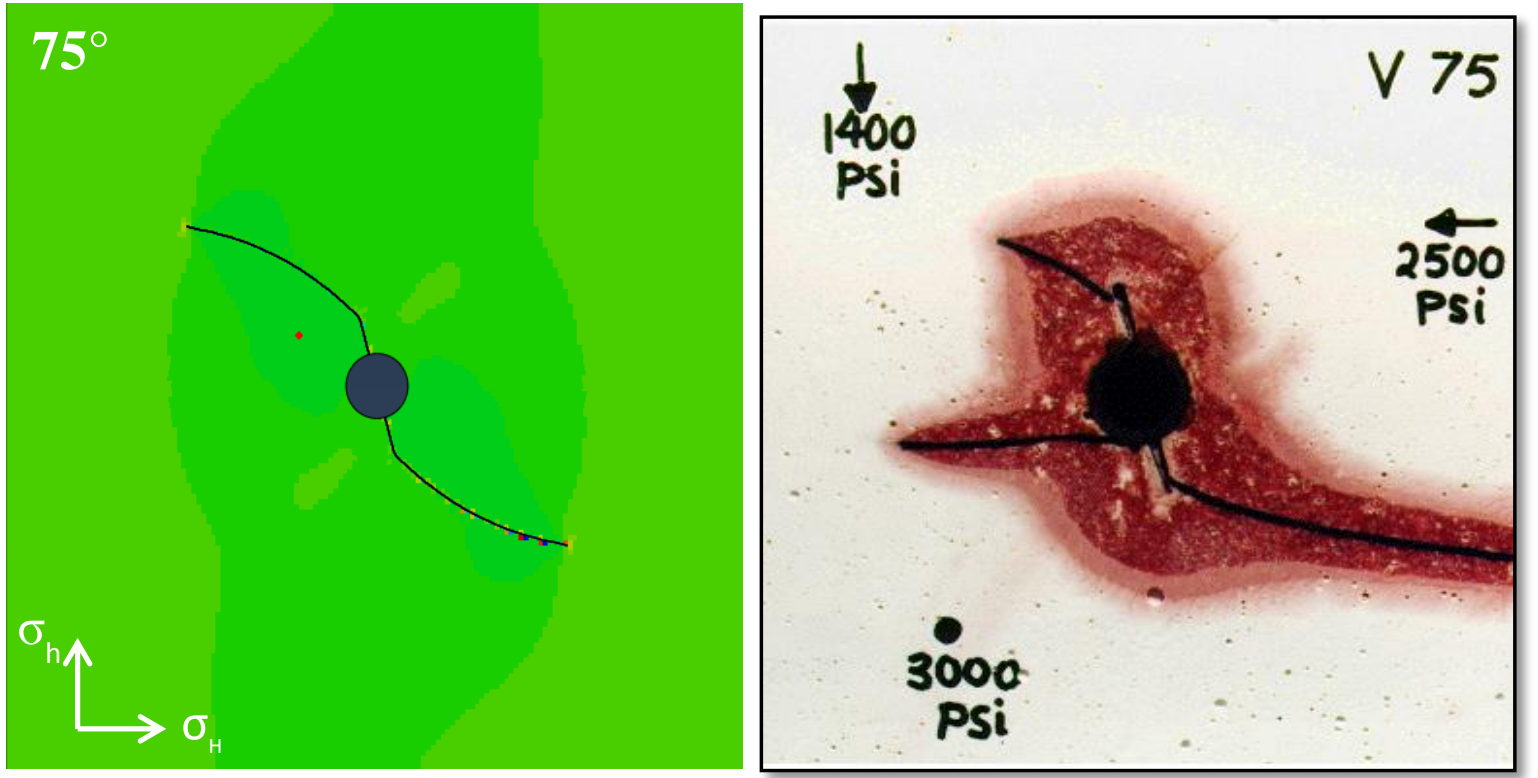

Figure 5.4: Fracture reorientation at $\theta=75^{\circ}$ and comparison to experimental model 

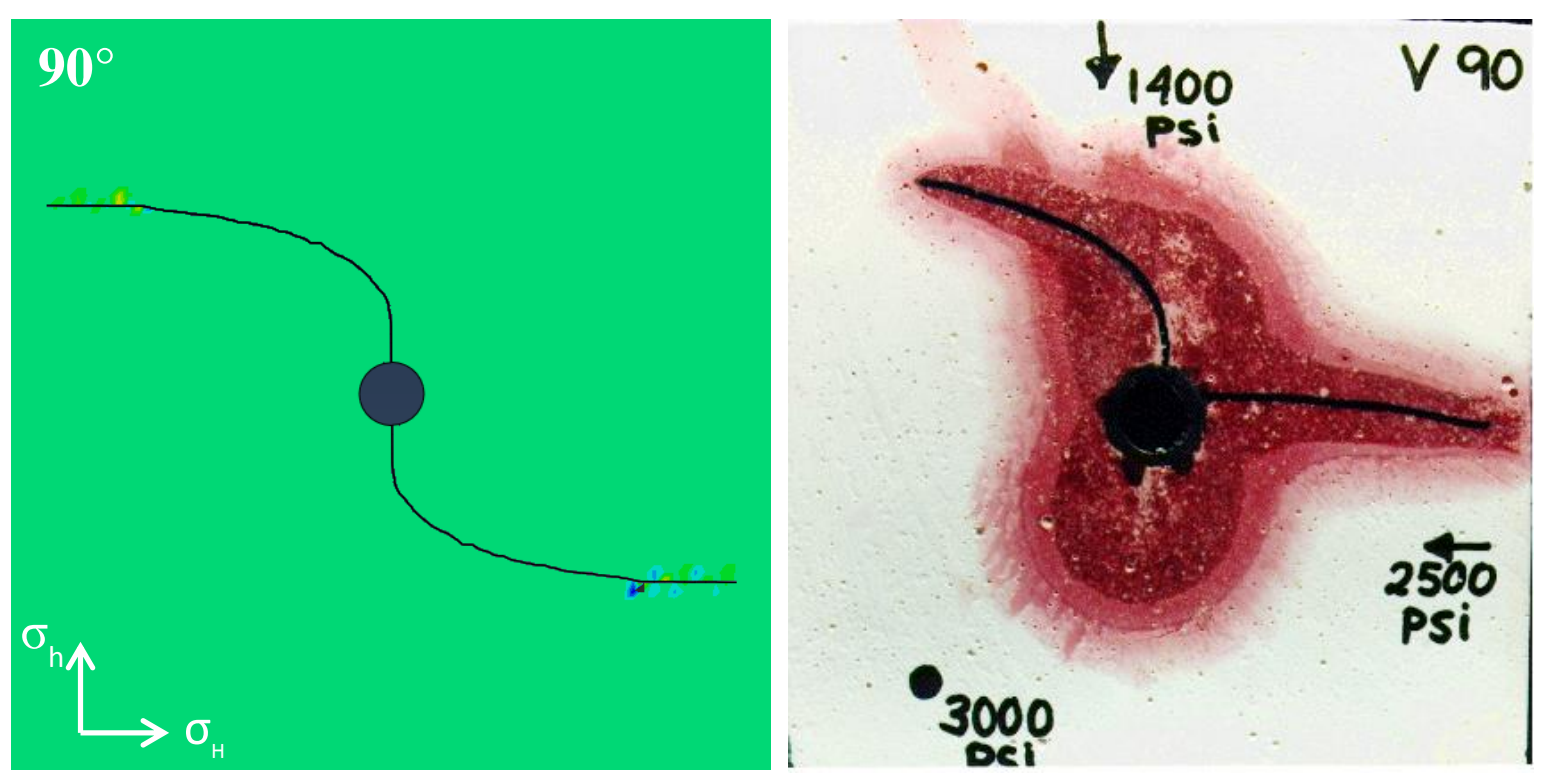

Figure 5.5: Fracture reorientation at $\theta=90^{\circ}$ and comparison to experimental model

As illustrated in figure 5.1 to 5.5 , there is a very good match between the results from the numerical model in this study and the experimental models in terms of fracture reorientation pattern. This gives a confidence in the model to perform further simulation. It should be remembered that the experimental study includes a cement layer behind the casing and in some cases, including $75^{\circ}$ and $90^{\circ}$, injected fluid has breached through the micro-annulus fractures in the cement to find a shorter path toward PFP. Since the numerical model is an ideal model without defect and the effect of loose cement and casing are not included, that phenomena will not happen and fractures initiate from perforations only.

\subsection{Reorientation Radius}

As fracture propagates away from wellbore, the near wellbore effects are diminished and fracture reorients to propagate in the preferred direction. The reorientation pattern is affected by stress distribution around wellbore. It is estimated that drilling a well will alter initial stress around wellbore over a distance of about 2-3 times the wellbore radius. Therefore, it takes some distance for fracture to exit the shadow of this stress change. The distance from wellbore to the point where fracture is completely 
aligned with PFP or perpendicular to minimum horizontal stress is called reorientation radius. This distance varies with orientation angle and is larger for more deviated perforations. Table 5.1 summarizes the results of a set of simulations where the reorientation has been measured for different orientation angles. Also these results are illustrated in figure 5.6.

Table 5.1: Reorientation Radius for different Perforation Angles

\begin{tabular}{|c|c|}
\hline $\begin{array}{c}\text { Orientation Angle } \\
\text { (Degrees) }\end{array}$ & $\begin{array}{c}\text { Reorientation Radius } \\
\left(\mathbf{x} \mathbf{r}_{\mathbf{w}}\right)\end{array}$ \\
\hline 0 & 0 \\
\hline 15 & 1.2 \\
\hline 30 & 1.8 \\
\hline 45 & 4 \\
\hline 60 & 5 \\
\hline 75 & 5.5 \\
\hline 90 & 7.5 \\
\hline
\end{tabular}

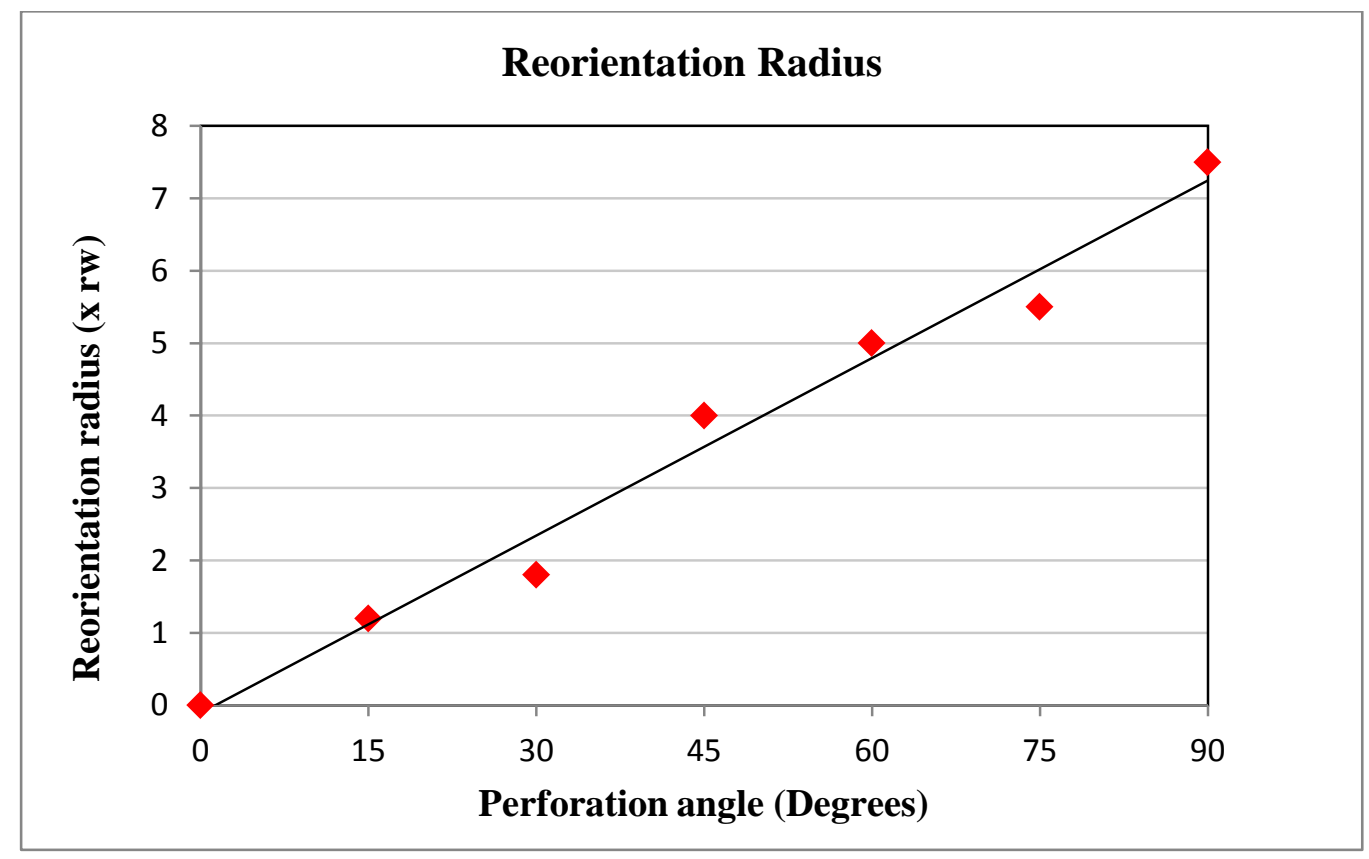

Figure 5.6: Effect of orientation angle on reorientation radius

The reorientation radius has been estimated graphically, but as expected shows an increasing trend when the orientation angle increases. This is due to the effect of stress 
distribution around wellbore. As the figure 5.7 shows, tangential stress around wellbore is increasing with increasing $\theta$. This is also the case at any radius around wellbore. Therefore, we expect the near wellbore effects to extend farther as $\theta$ deviate from the direction of maximum horizontal stress which implies that in higher angles, it takes longer for the fracture to exit the near wellbore stress regime.

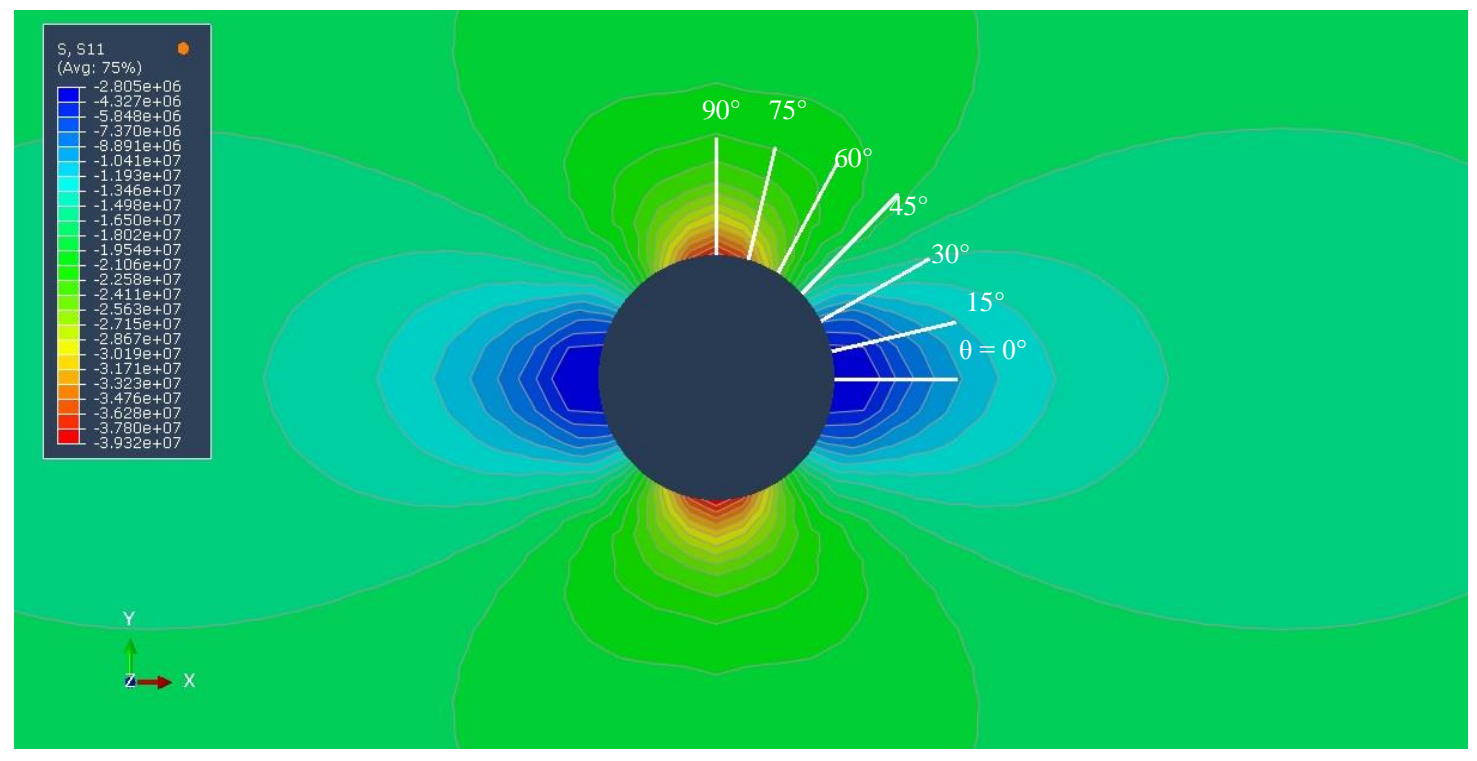

Figure 5.7: Fracture reorientation affected by stress distribution around wellbore

\subsection{Break-Down Pressure}

The previous simulation set was run several times to obtain the breakdown pressure. Different pressures were applied to the model to find the smallest pressure at which fracture start to propagate. The results are presented in table 5.2 and plotted on figure 5.8.

As the results of this sensitivity analysis shows, the breakdown pressure is increasing as the perforation angle increases. This is due to the fact that higher pressure is required to overcome the tangential stress at wellbore in higher angles. Tangential stress for a wellbore of radius $\mathrm{r}=0.00635 \mathrm{~m}$ under the maximum and minimum horizontal stress of $2500 \mathrm{psi}$ and $1400 \mathrm{psi}$ is plotted in figure 35. At the tip of perforation $\left(R=2 r_{w}\right)$, this stress are increasing as plotted in figure 5.9 and 5.10. 
Table 5.2: Breakdown pressure vs perforation angle

\begin{tabular}{|c|c|}
\hline $\begin{array}{c}\text { Orientation } \\
\text { Angle } \\
\text { (Degrees) }\end{array}$ & $\begin{array}{c}\text { Breakdown } \\
\text { Pressure } \\
\left(\mathbf{x} \mathbf{1 0}^{7} \text { pa) }\right.\end{array}$ \\
\hline 0 & 3.2 \\
\hline 15 & 4.2 \\
\hline 30 & 4.8 \\
\hline 45 & 5.4 \\
\hline 60 & 6.8 \\
\hline 75 & 6.6 \\
\hline 90 & 7.2 \\
\hline
\end{tabular}

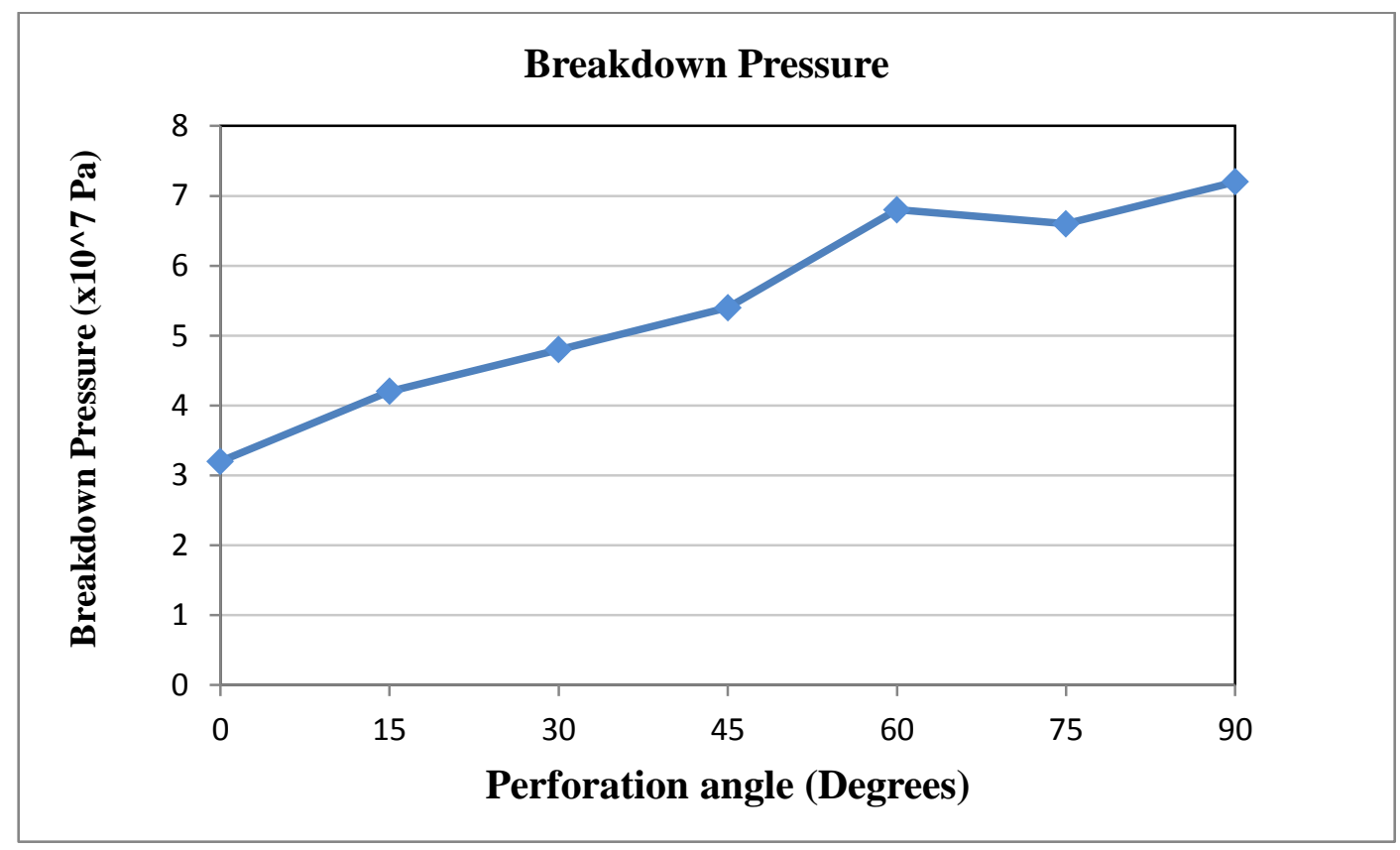

Figure 5.8: Change in break down pressure with perforation angle

It should be noted that the plot in figure 5.10 is based on equation 27 in chapter 4 which shows tangential stress around wellbore as a function of $r$ and $\theta$. 
Texas Tech University, Jay Sepehri, May 2014

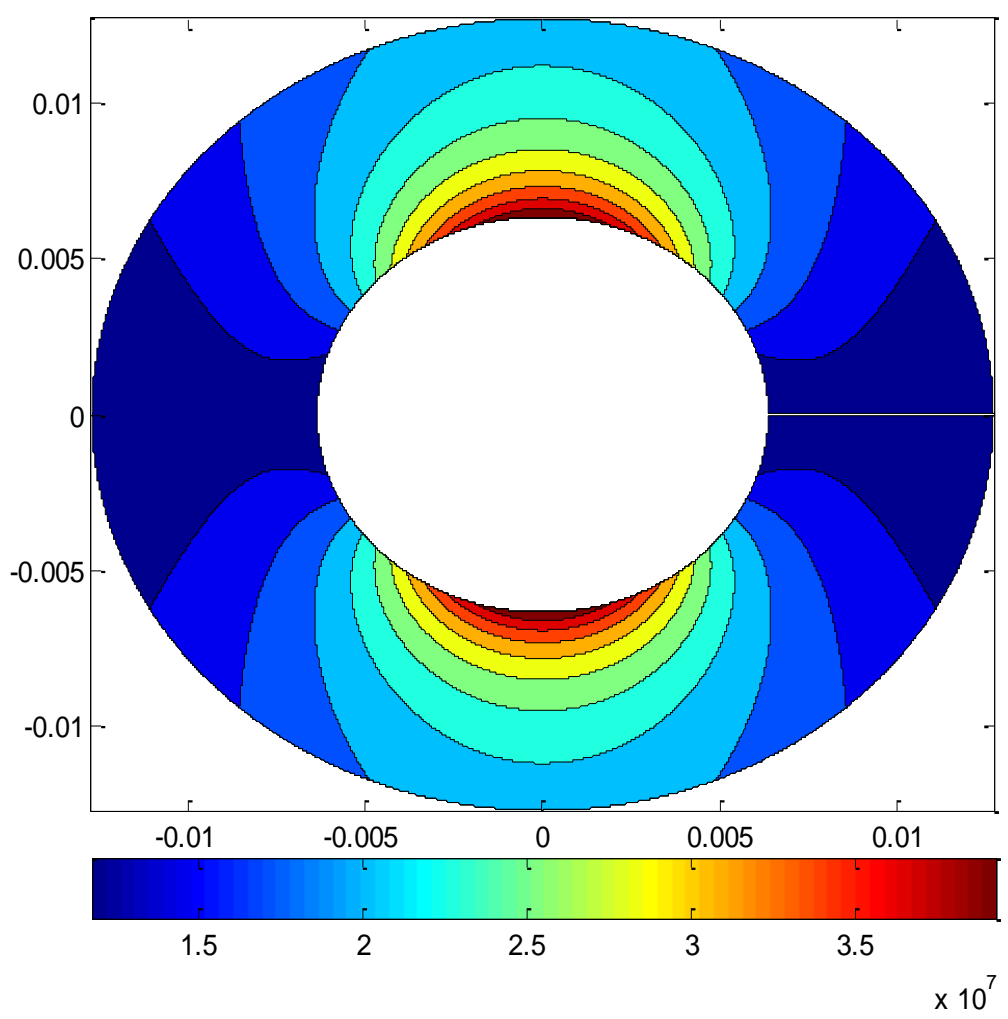

Figure 5.9: Tangential stress around wellbore increases with increasing angle

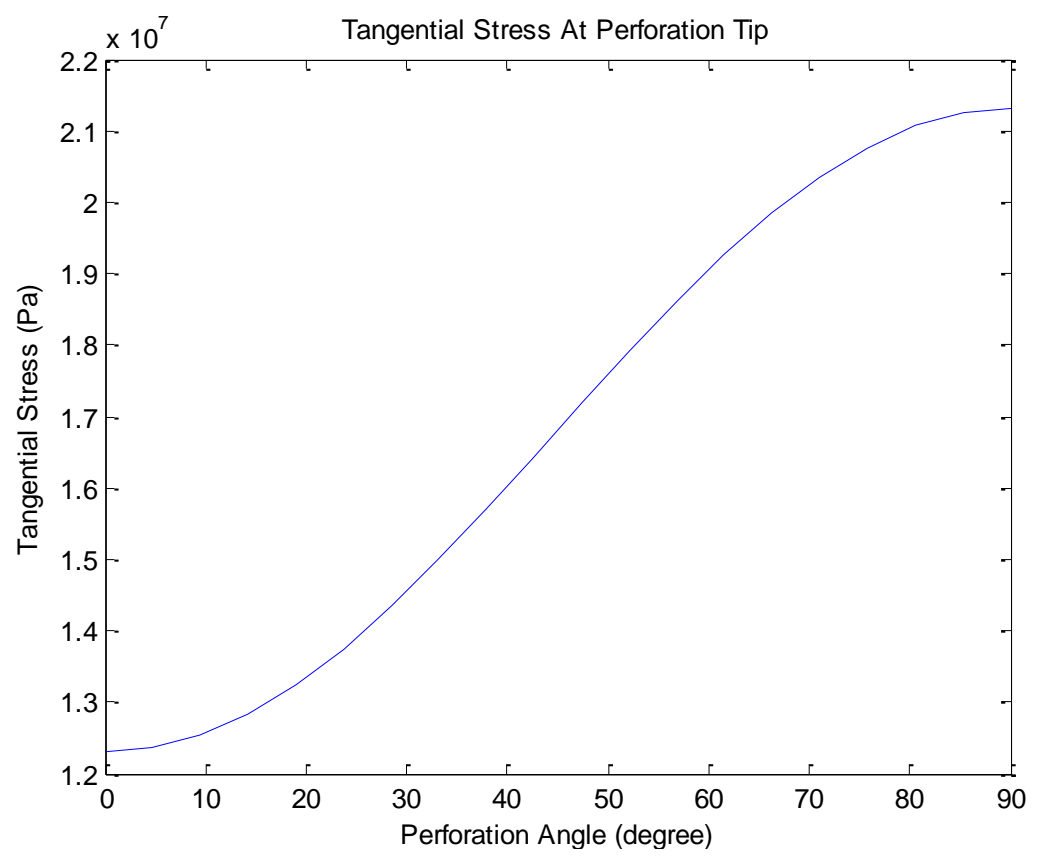

Figure 5.10: Tangential Stress at Perforation tip for different angles $\left(L=r_{w}\right)$ 


\subsection{Initial Perforation Length}

Perforation length is one is the main parameters to be considered when designing hydraulic fracturing from cased holes. The typical perforating guns have penetrations of 1 to 30 inches. The length of the actual perforation downhole is a function of the standoff of the perforating gun from the casing. Less standoff means a longer perforation tunnel, while more standoff results in a shorter perforation tunnel. For this study, since we are dealing with a small scale model, perforation length is limited to be in the range of one quarter to twice wellbore radius.

Result of simulation for different perforation lengths are compared in figure 5.11. Perforations are all $45^{\circ}$ and other parameters are kept constant, except the perforation length changing from $0.25 . \mathrm{r}_{\mathrm{w}}$ to $2 . \mathrm{r}_{\mathrm{w}}$.

The initial perforation path affects the direction of fracture growth. As can be observed from figure 5.11, the longer the perforation length, the longer it takes for the fracture to reorient. This argument is true for the cases where fractures are only allowed to initiate from the tip of perforations. If casing with cement with low bounding property were considered in the model and fracture could propagate through microfractures in the cement, the result would have been different. In that case, fracture may choose to propagate through those microfractures due to the friction loss and pressure drop in long perforations.

Figure 5.12 compares the tangential stress for different perforation length. As perforation length increases (larger $\mathrm{L} / \mathrm{r}_{\mathrm{w}}$ ), perforation tip is located at areas with lower tangential stress, which means stress to overcome at the tip of perforation is lower and this results in fracture to propagate easier. 
Texas Tech University, Jay Sepehri, May 2014
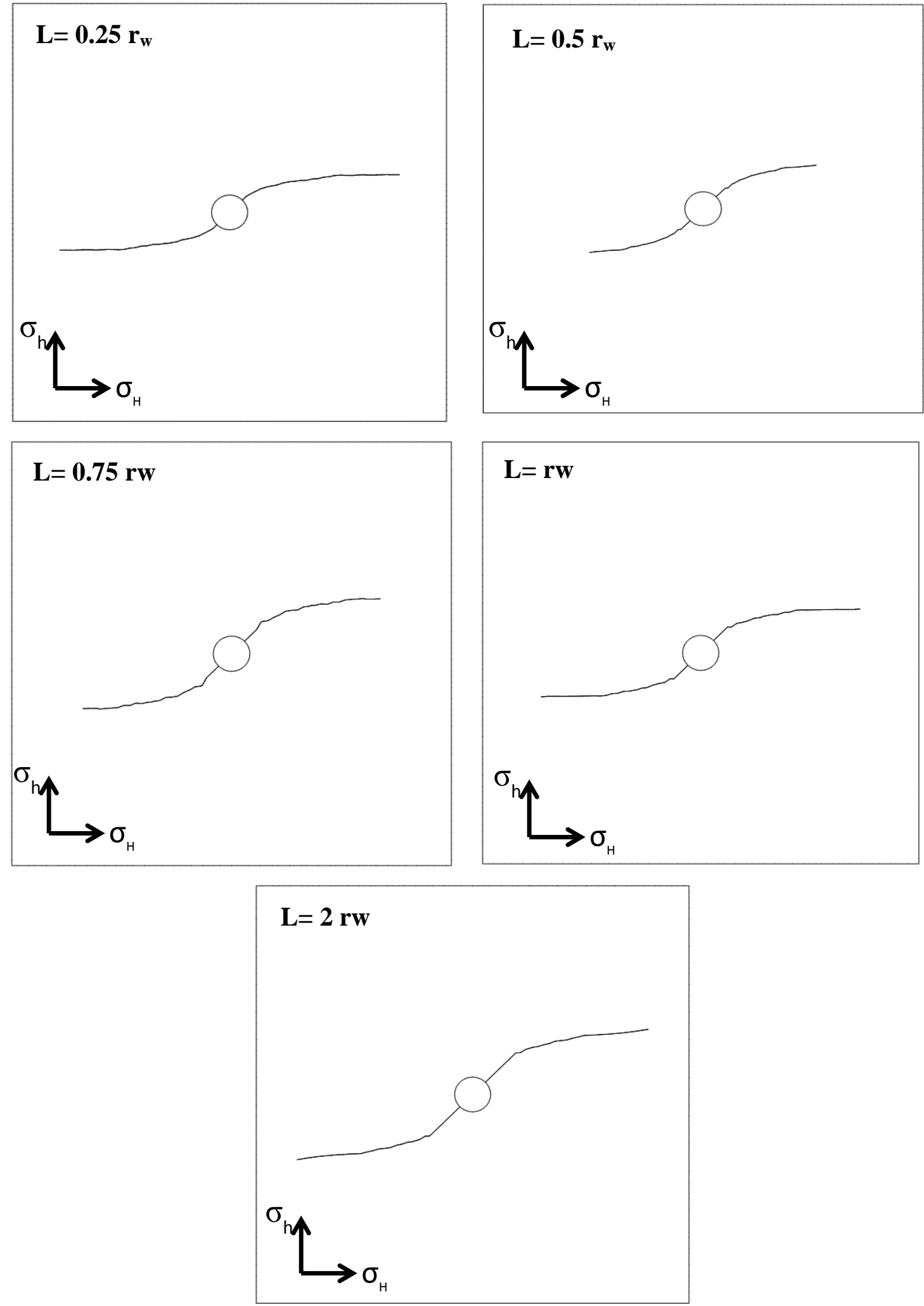

Figure 5.11: Effect of perforation length on fracture reorientation 


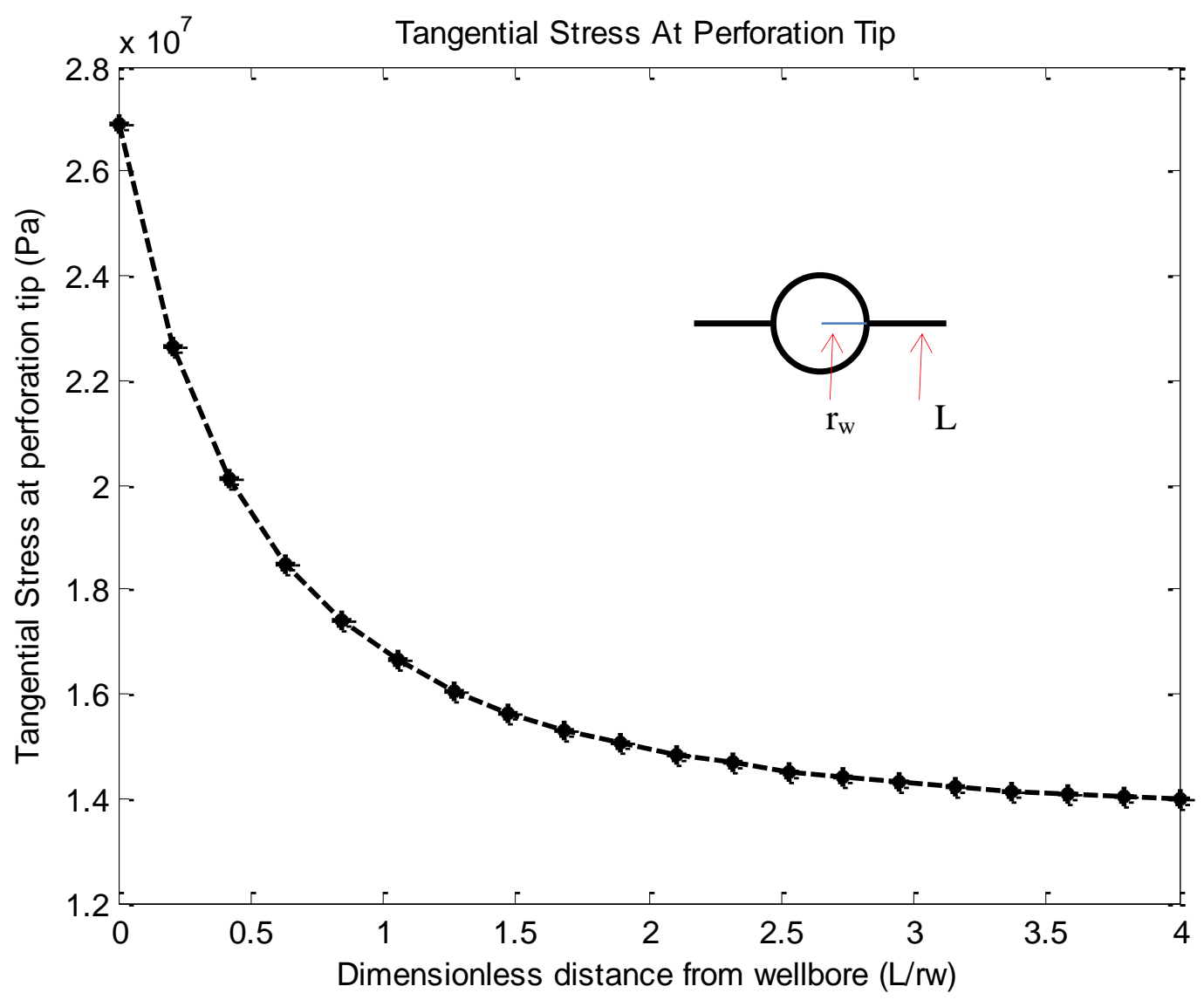

Figure 5.12: Variation of tangential stress with perforation length

\subsection{Mechanical Properties}

Young modulus is one of the mechanical properties examined in this model to observe its effect on fracture propagation. Young's modulus has small effect on initial stress distribution, but has significant effect on fracture propagation pattern. Increasing Young's modulus increases the reorientation radius. By increasing Young's modulus from $0.2 \times 10^{6}$ psi to $6 \times 10^{6} \mathrm{psi}$, reorientation radius has increased 1.5 fold. The effect of young modulus is presented graphically in figures 5.13 and plotted on figure 5.14. 


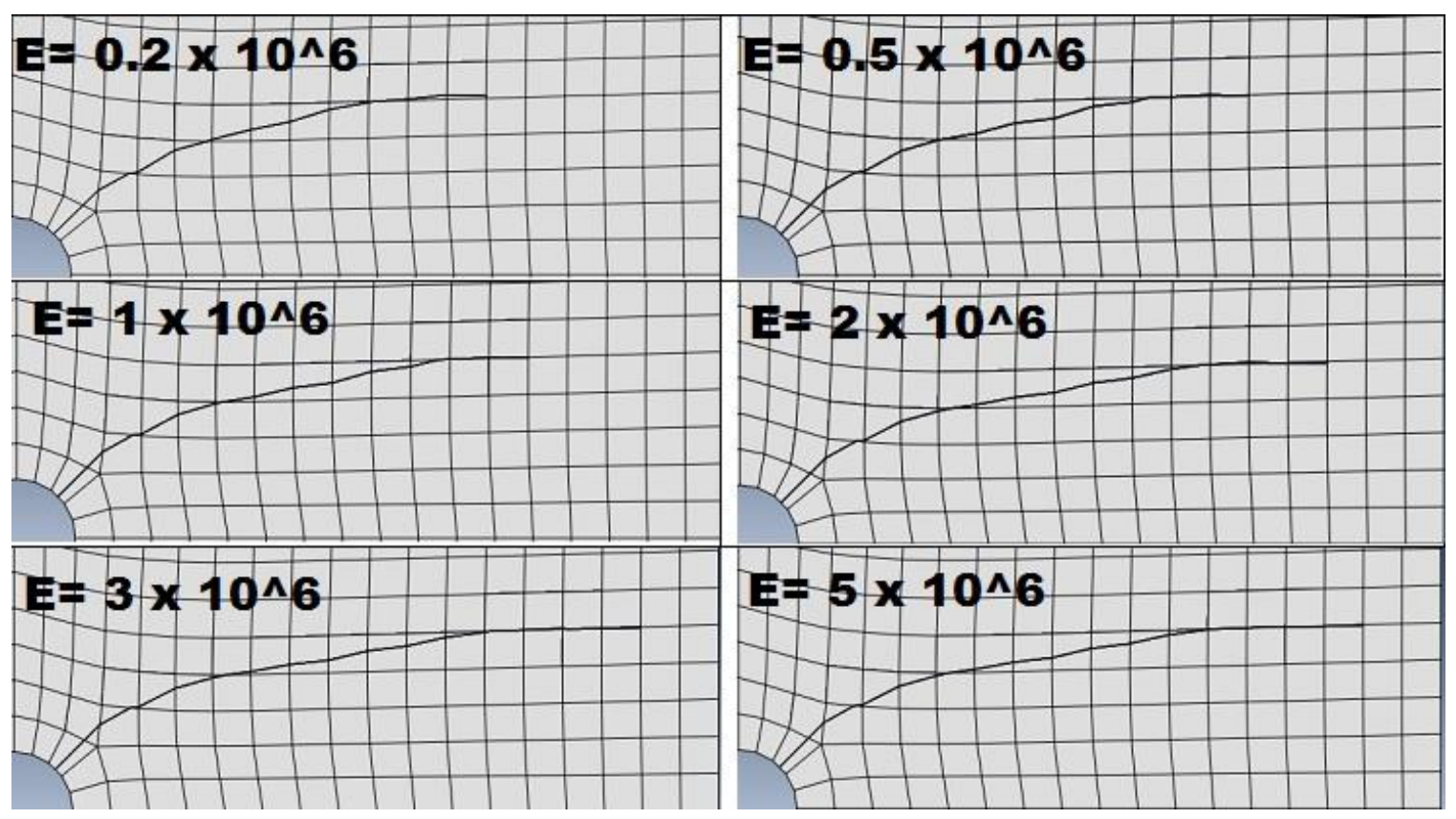

Figure 5.13: Effect of Young's Modulus on fracture propagation pattern $\left(\theta=45^{\circ}\right)$

\section{Effect of Young's Modulus on Fracture Reorientation}

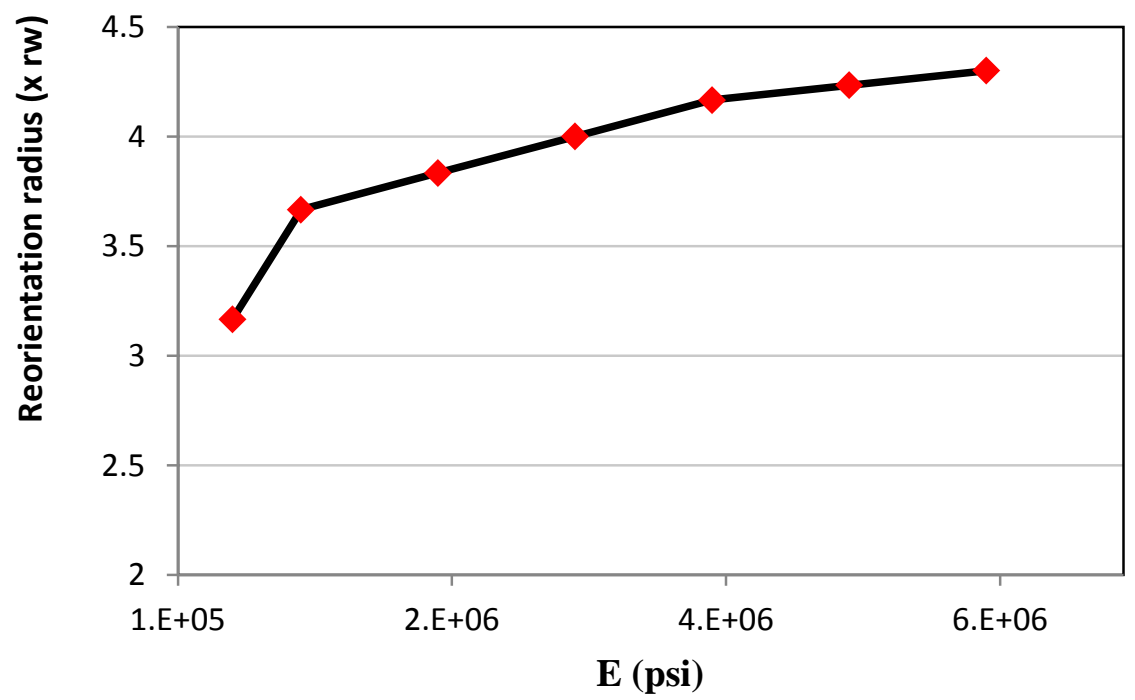

Figure 5.14: Young's Modulus effect on reorientation radius $\left(\theta=45^{\circ}\right)$ 


\subsection{Effect of Stress Anisotropy}

Stress anisotropy, the difference between maximum and minimum horizontal stress plays and important role in fracture propagation pattern. Two sets of simulation were run to observe the effect of stress anisotropy. In the first set, simulations are run for different horizontal stresses at a fixed orientation angle of 45 degrees and the results are displayed in figures 5.15. As stress anisotropy decrease (increasing $S_{h}$ ) there is an increasing trend in reorientation radius.
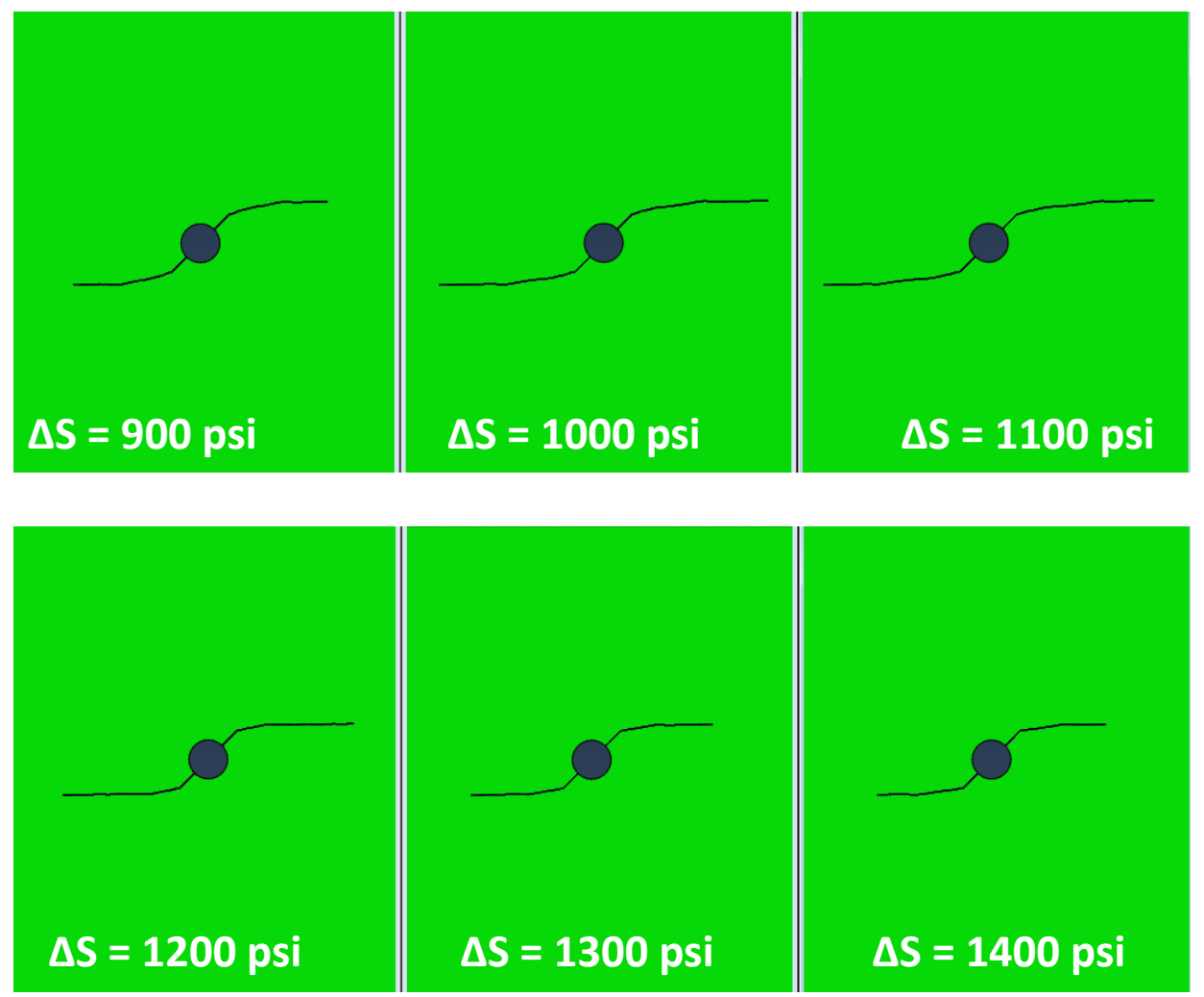

Figure 5.15: Effect of stress anisotropy on fracture propagation pattern $\left(\theta=45^{\circ}\right)$

The next set of simulation to observe the effect of stress anisotropy is to rerun simulations for different angles with a new minimum horizontal stress of 1000 psi. Reorientation pattern are then compared for each angle, side by side. Fracture propagation patterns for different anisotropies are compared in figures 5.16 and 5.17. 
This set of simulation confirms that increasing stress anisotropy will result in a faster reorientation of fracture in a shorter distance. For more deviated angles, this effect is more visible especially in $75^{\circ}$ and $90^{\circ}$.

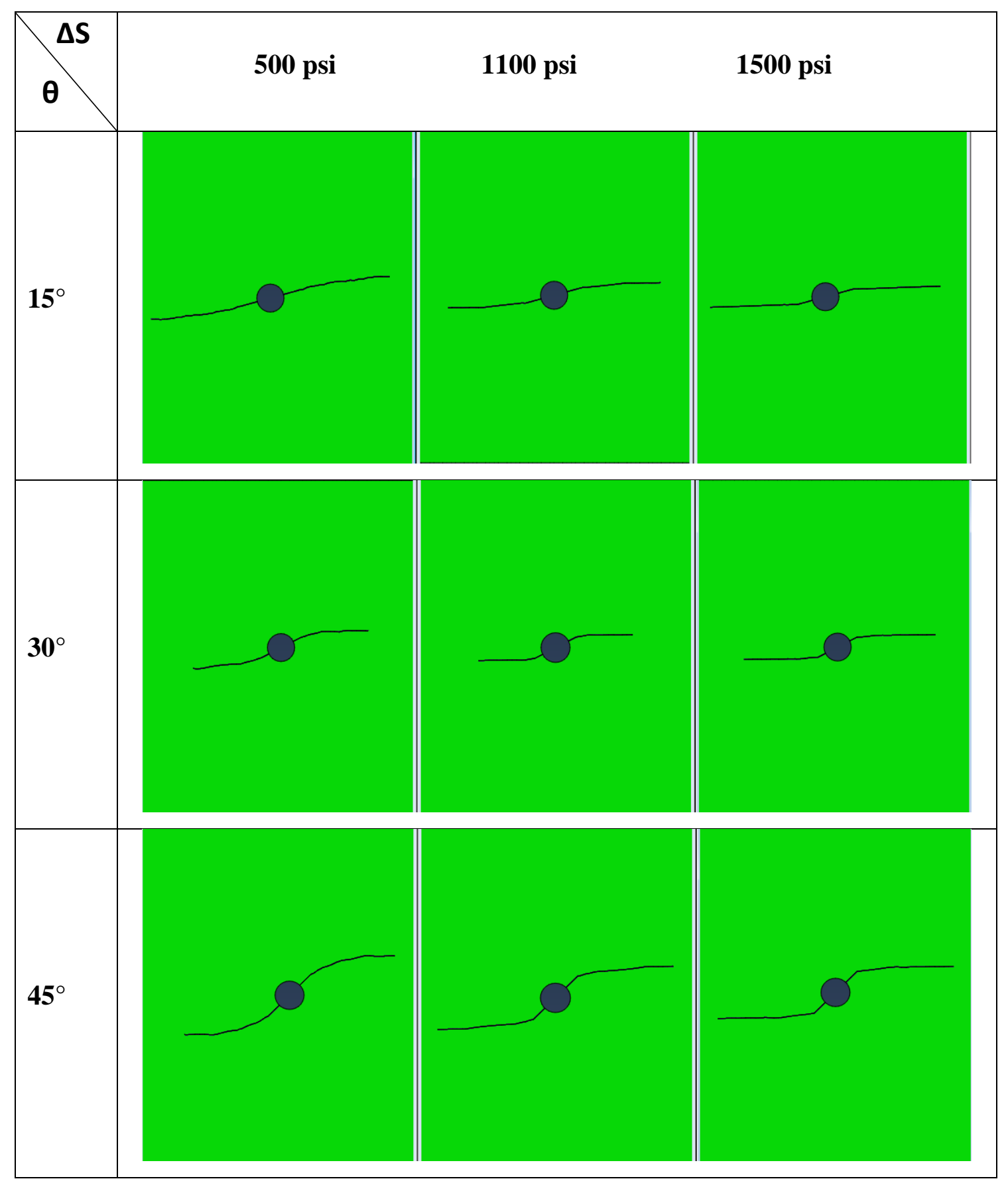

Figure 5.16: Effect of stress anisotropy on fracture propagation $\left(15^{\circ}-30^{\circ}-45^{\circ}\right)$ 


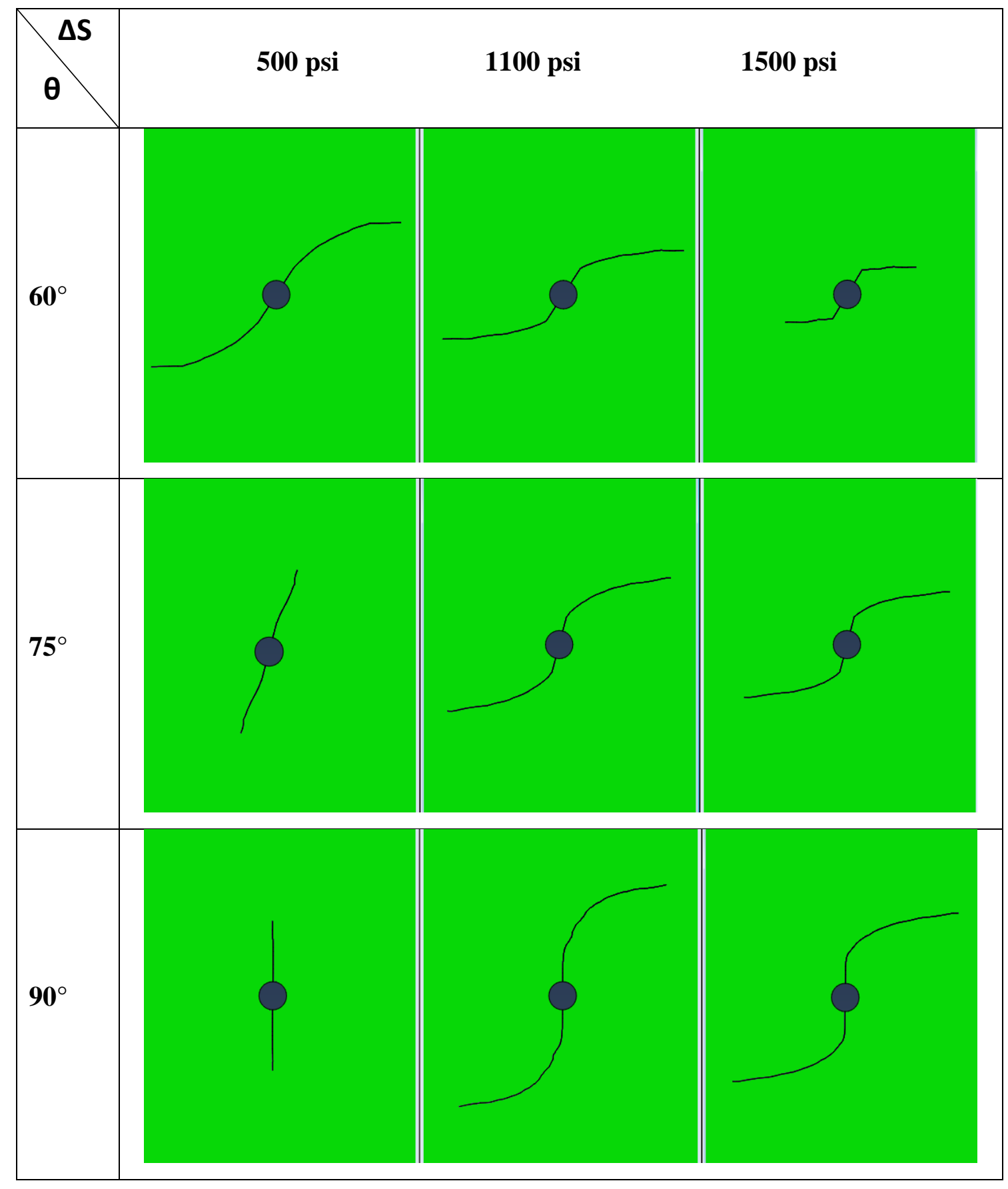

Figure 5.17: Effect of stress anisotropy on fracture propagation $\left(60^{\circ}-75^{\circ}-90^{\circ}\right)$ 
The results from this simulation suggest that in formation with higher stress anisotropy, role of perforation orientation becomes more important. Higher anisotropy results in rapid change in fracture direction and this means increasing tortuosity and limiting fracture width. Therefore, in higher anisotropy, there is a smaller tolerance on perforation deviation angle. This should be considered when perforating for hydraulic fracture treatment.

\subsection{Varying Rock Property}

In this scenario, rock mechanical property of some section of the rock sample was considered to be different and simulations were run again. Result is presented in figure 5.18 .
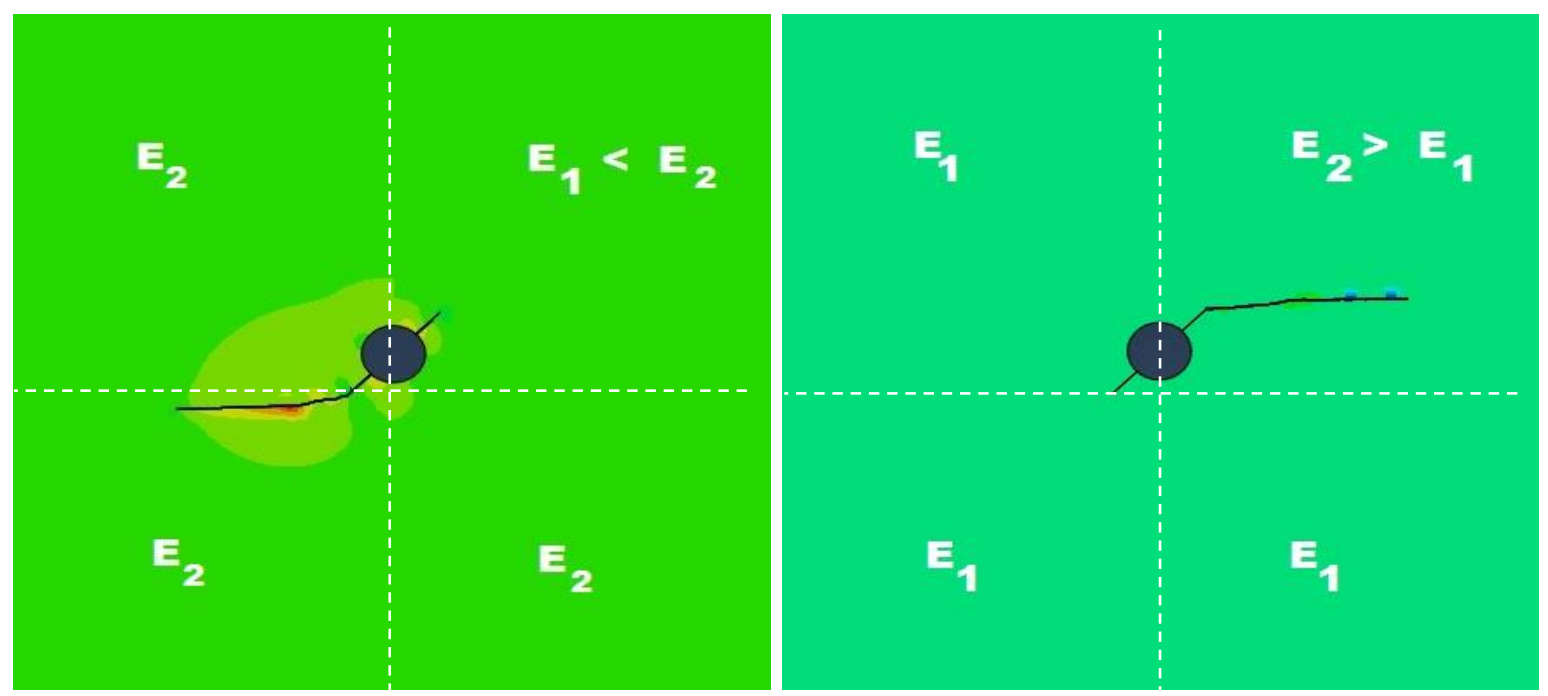

Figure 5.18: Effect of varying rock properties

Fracture from perforation in stiffer rock propagates and reorient before the perforation in the opposite side even initiate a fracture. The result in figure 5.18 suggest that perforation is positioned at more frackable part of rock has higher chance of initiating and propagating a fracture and formations with varying property may develop asymmetric one wing fracture.

\subsection{Effect of Competing Perforation}

All simulations in previous sections are done with two perforations positioned in opposite sides of the wellbore which is $180^{\circ}$ phasing perforation configuration. Other 65 
configurations such as $120^{\circ}, 60^{\circ}$ are also applied in the field. They are of course on a spiral or helix configuration. Here in this model all the perforations are applied on a horizontal 2-D model. The results for two perforations with smaller phasing are presented in figure 5.19 .

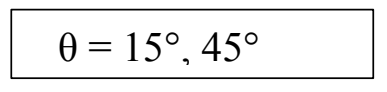

$\theta=30^{\circ}, 60^{\circ}$
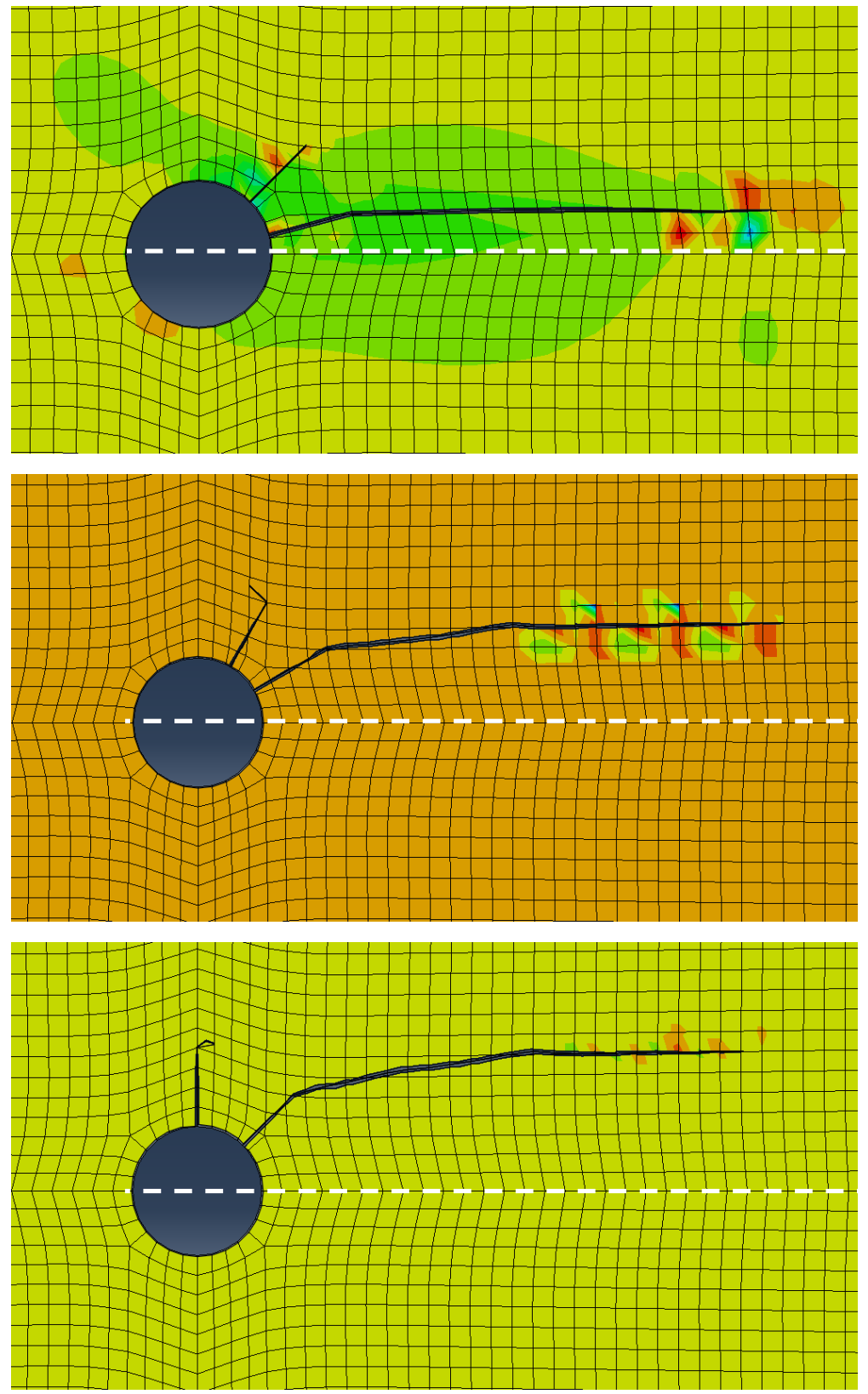

Figure 5.19: Effect of two competing perforations 
When two or more perforations are on a plane (assuming perforation at the same depth instead of on a helix configuration) there is competition between the fractures to propagate.

As figures 5.19 demonstrate, when two perforations exist $\left(15^{\circ} / 45^{\circ}\right.$ or $30^{\circ} / 60^{\circ}$ or $45^{\circ} / 90^{\circ}$ ), fracture will propagate from the perforation positioned at smaller angle from PFP. This can be because of easier fracture initiation and propagation from less deviated perforations. In reality, fracture initiated from perforations at close angle, may link up and form one fracture which would be in the direction of less deviated perforation.

Also, as can be observed in the case of $30^{\circ} / 60^{\circ}$ perforations, the initiated fracture propagate away from each other due to stress interference which probably result in early screen out of perforation with higher deviation angle. The same discussion can be made for three competing fracture in $120^{\circ}$ phasing as depicted in figure 5.20.

The results obtained from simulating competing perforations, can have many application in designing perforation for hydraulic fracturing. In cases where perforation are not aligned with PFP due to lack of information on PFP orientation or due to operational limitations, using the concept of two or more competing perforations can help increase the chance of developing fracture in PFP direction and avoiding tortuosity. Also by decreasing perforation phasing, there are more chances to have more fracture in an orientation close to PFP. Although there are always concerns about casing stability in lower phasing angles or higher shot densities, the idea of competing perforation can still be used by increasing operational efficiency. 

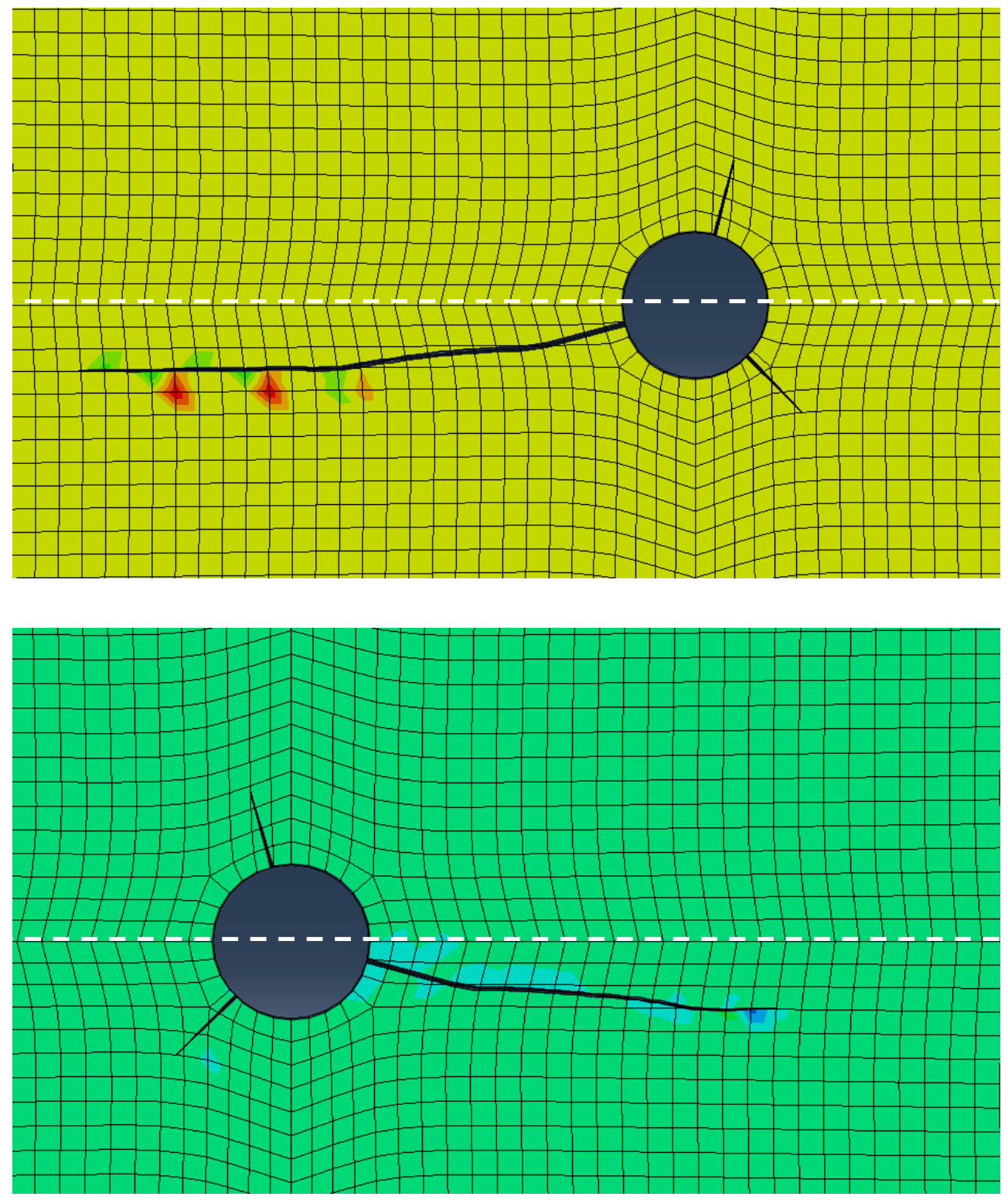

Figure 5.20: Three competing perforations at $120^{\circ}$ phasing

It should be noted that perforations cannot be placed too close to each other. For example, positioning two perforations at $30^{\circ}$ and $45^{\circ}$ will result in perforation breakdown as the results in figure 5.21 suggests. In this case, the stress interference from two perforations will force fracture propagating away from each other which would not be a favorable phenomenon. 

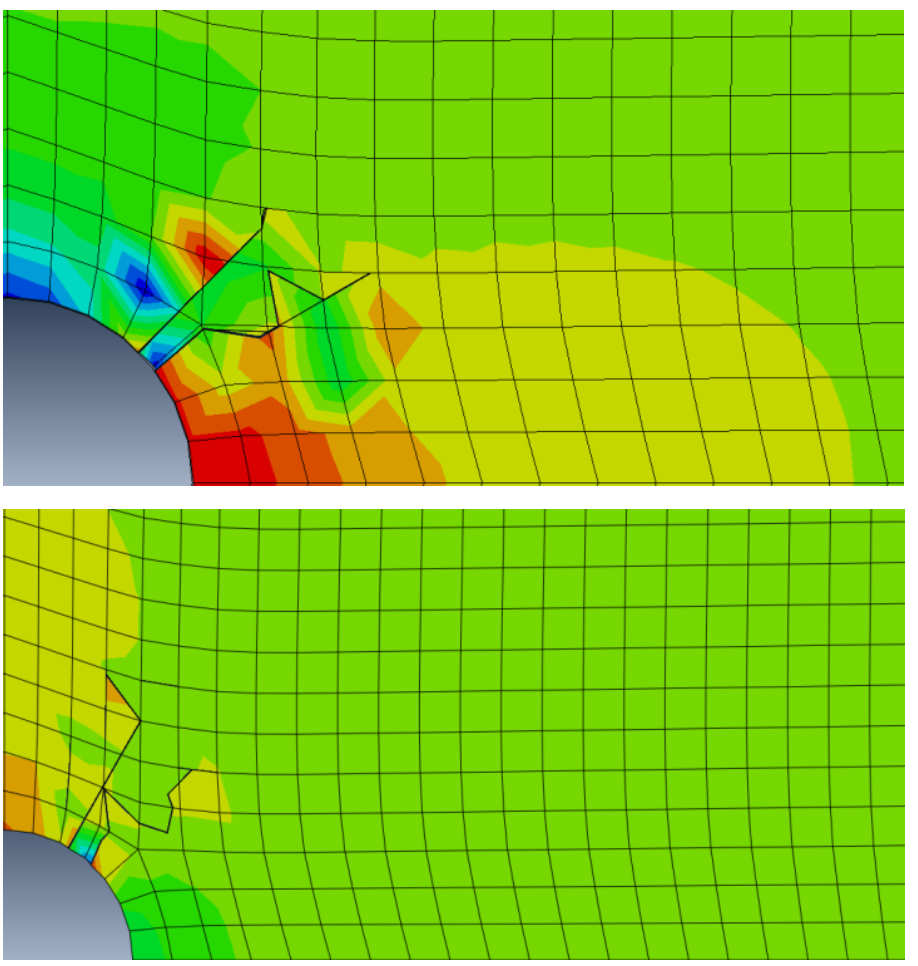

Figure 5.21: Two competing perforations positioned at $15^{\circ}$

Even if one of these perforations could result in a fracture propagating, as shown on figure 5.22, the resulted tortuosity can be very severe around wellbore which eventually reduce in wellbore production performance.

$\theta=30^{\circ}, 50^{\circ}$

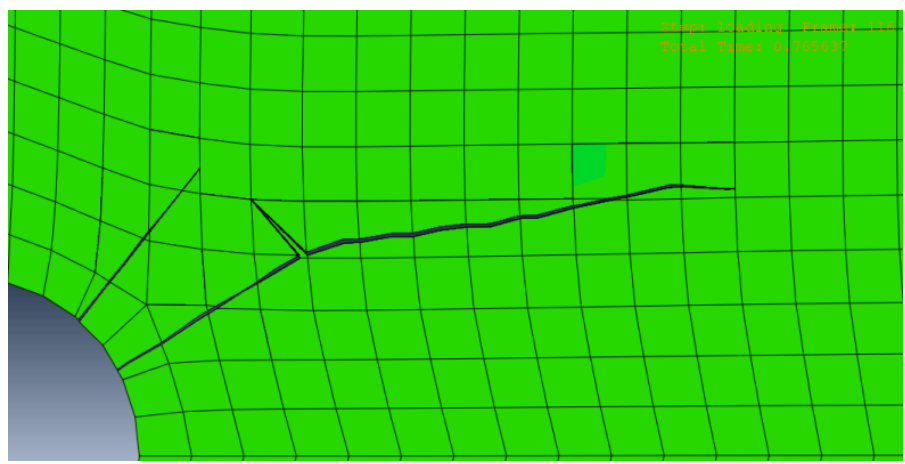

Figure 5.22: Two competing perforations at $30^{\circ}$ and $50^{\circ}$ 


\section{Chapter 6}

\section{Conclusions and Recommendations}

A 2-D numerical model was developed using XFEM capability of software Abaqus, to investigate hydraulic fracture propagation from oriented perforations. This model makes it possible to conduct numerical analysis on parameters involved in hydraulic fracture treatment from perforated wellbores. The main goal in this study was to highlight and verify the effect of parameters considered to be affecting this process as well as emphasizing on promising capabilities of XFEM in solving hydraulic fracturing simulation problems. Based on the findings in this study verified with available experimental works, following conclusions were drawn:

1. Perforation orientation angle ( $\theta$, measured from Preferred Fracture Plane) has strong effect on fracture propagation pattern. This effect was quantified in this study. Fractures propagated from more deviated perforations require higher breakdown pressure and tend to reorient to PFP direction over larger distances.

2. Increasing perforation length will increase fracture reorientation radius.

3. Changing formation Young's Modulus has small effect on initial stress distribution around wellbore, but directly affect fracture reorientation radius.

4. Horizontal stress anisotropy, the difference between min and max horizontal stress, affects fracture propagation pattern. In formations with higher stress anisotropy, fractures propagated from perforations reorient over a shorter distance. This effect is stronger for more deviated perforations.

5. Perforation positioned at the part of formation with larger Young's modulus has higher chance of initiating and propagating a fracture. Therefore rock with 
varying property may develop asymmetric one-wing fracture, even if perforated at $180^{\circ}$ phasing.

6. There is competition between the perforations with different orientation angle to develop hydraulic fracture. In these cases fracture tends to propagate from less deviated perforations.

7. In the case of competing perforations, the more deviated one may develop a short fracture, propagating away from the other one due to stress interference and/or cease to propagate over a short distance which will result in perforation screenout.

8. Stress interference between two perforations positioned close to each other, results in either perforation breakdown or fracture propagating away from each other.

Based on the experience and insights gained during this study, following recommendations for design of hydraulic fracture treatment from perforated wells are offered:

1. Since horizontal stress anisotropy plays an important role in fracture reorientation radius and consequently tortuosity, in formation with high stress anisotropy, care must be taken to identify preferred fracture plane and avoid perforation in orientations highly deviated from this plane.

2. Results from competing perforations analysis suggest that this technique can be applied when there is high uncertainty about the direction of maximum and minimum horizontal stresses. This technique can be applied through increasing shots density or a new design of perforation gun to apply multiple perforations at the same depth.

Also, the following research directions are proposed to further understand the hydraulic fracture propagation from oriented perforations: 
3. Although fracture propagation pattern were confirmed to match results from limited experimental studies available, other simulation results including the effect of varying rock property, perforation length, perforation phasing and competing perforations should be tested in an experimental model to be completely verified.

4. 2-D model was considered in this study because of simplicity, saving run-time and being able to compare to experimental study results. Performing these simulations on a 3-D and large scale model can give better insight into hydraulic fracture propagation. Also 3-D model can provide the opportunity to study fracture height propagation, effect of multilayers and helix configuration of perforations.

5. Since many parameters observed to be important in hydraulic fracturing treatment design from perforations, effect of these parameters can be further studied specially for the cases with high horizontal anisotropy and varying rock properties. 


\section{References}

Abass, H. H., Hedayati, S. and Meadows D. L. 1996. "Nonplanar Fracture Propagation From a Horizontal Wellbore : Experimental Study.” SPE Paper 24823.

Abass, H. H., Meadows. D. L., Brumley, D. L., Hedayati, S. and Venditto, J. J. 1994. “Oriented Perforations - A Rock Mechanics View.” SPE Paper 28555.

Almaguer, J., Manrique, J., Wickramasuriya, S., López-de-cárdenas, J., May, D., Mcnally, A. C. and Sulbaran, A. 2002. "Oriented Perforating Minimizes Flow Restrictions and Friction Pressures during." Oilfield Review 14 (1): 16-31.

Aud, W. W., Wright, T. B., Cipolla, C. L., Harkridar, J. D., and Haneen, J. T. 1994. "The Effect of Viscosity on Near-Wellbore Tortuosity and Premature Screenouts." SPE Paper 28492, 305-317.

Bakala, M. "Fracture Propagation in Sediment-like Materials." PHD Dissertation, University of Oklahoma, 1997.

Behrmann, L. A., and Nolte, K. G. 1999. "Perforating Requirements for Fracture Stimulations.” SPE Paper 59480, 14 (4): 228-234.

Belytschko, T., Areias, P., Wang, H. W. and Xu, J. X. 2005. "The Extended Finite Element Method for Static and Dynamic Crack Propagation.” International Journal of Numerical Methods in Engineering. 63, 760-788.

Benzeggagh, M. L., and Kenane, M. 1996. "Measurement of Mixed-Mode Delamination Fracture Toughness of Unidirectional Glass/Epoxy Composites with Mixed-Mode Bending Apparatus." Composites Science and Technology 56 (4): 439-449.

Bonner, S., Bagersh, A., Clark, B., Dajee, G., Dennison, M., Hall, J.S., Jundt, J., Lovell, J., Rosthal, R. and Allen, D. 1994. "A New Generation of Electrode Resistivity Measurements for Formation Evaluation While Drilling." Transactions of the SPWLA 35th Annual Logging Symposium. Tulsa, OK. 
Bunger, A. P., Jeffrey, R. G. and Detournay, E. 2004. “Toughness-Dominated NearSurface Hydraulic Fracture Experiments." $6^{\text {th }}$ North America Rock Mechanics Symposium (NARMS)

Burlayenko, V. N. and Sadowski, T. 2008. "FE Modeling of Delamination Growth in Interlaminar Fracture Specimens.” Budownictwo I Architektura 2: 95-109.

Chen, M., Jiang, H., Zhang, G. Q. and Jin, Y. 2010. “The Experimental Investigation of Fracture Propagation Behavior and Fracture Geometry in Hydraulic Fracturing through Oriented Perforations." Petroleum Science and Technology 28 (13) (July 12): 12971306.

Chen, Z. 2013. “An ABAQUS Implementation of the XFEM for Hydraulic Fracture Problems." International Conference for Effective and Sustainable Hydraulic Fracturing, 725-739. Brisbane.

Cleary, M. P., Johnson, D. E., Kogsbell, H. H., Owens, K. A., Perry, K. F., De Pater, C. J., Stachel, A., Schmidt, H., and Tambini, M. 1993. "Field Implementation of Proppant Slugs to Avoid Premature Screen-Out of Hydraulic Fractures with Adequate Proppant Concentration." SPE Paper 25892.

Dahi-Taleghani, A. and Olson, J. E. 2011. "Numerical Modeling of MultistrandedHydraulic-Fracture Propagation: Accounting for the Interaction Between Induced and Natural Fractures.” SPE Paper 124884: 575-581.

Daneshy, A. A. 1973. "Experimental Investigation of Hydraulic Fracturing Through Perforations." Journal of Petroleum Technology: 1201-1206.

Dassault Systemes. “Abaqus 6.9 Overview.” Last Modified 2009. http://abaqusdoc.ucalgary.ca/v6.9/books/rnb/default.htm.

Dassault Systèmes. 2013. “Abaqus Documentation.” Dassault Systèmes. Providence, RI, USA. 
De Pater, C. J., Weijers, L., Savic, M., Wolf, K.H.A.A., van den Hoek, P. J. and Barr, D. T. 1994. "Experimental Study of Nonlinear Effects in Hydraulic Fracture Propagation." SPE Journal Paper 25893-PA.

Dolbow, J. "An Extended Finite Element Method with Discontinuous Enrichment for Applied Mechanics." PhD Dissertation, Northwestern University, 1999.

Duarte, C. A., Babuska, I. and Oden, J. T. 2000. "Generalized Finite Element Methods for Three-Dimensional Structural Mechanics Problems." Computers \& Structures, 77 (2): 215-232.

Elisa, P. 2011. "Virtual Crack Closure Technique and Finite Element Method for Predicting the Delamination Growth Initiation in Composite Structures" in Advances in Composite Materials-Analysis of Natural and Man-Made Materials, Edited by Dr. Pavla Tesinova.

El-rabaa, W. 1982. "Experimental Study of Hydraulic Fracture Geometry Initiated From Horizontal Wells.” SPE Paper 19720.

Fleming, M., Chu, Y. A., Moran, B. and Belytschko, T. 1997. "Enriched ElementFree Galerkin Methods for Crack Tip Fields.” International Journal for Numerical Methods in Engineering 40 (8): 1483-1504.

Gasser, T. C. and Hozapfel, G. A. 2005. "Modeling 3D Crack Propagation in Unreinforced Concrete Using PUFEM" Computer Methods in Applied Mechanics and Engineering 194 (25-26): 2859-2896.

Giner, E., Sukumar, N. and Taranc, J. E. 2008. "An Abaqus Implementation of the Extended Finite Element Method.” Engineering Fracture Mechanics. 76 (3), 347-368

Hallam, S. D., and Last, N. C. 1991. "Geometry of Hydraulic Fractures from Modestly Deviated Wellbores." SPE Paper 20656, 742-748.

Hanson, M. E., Anderson, G. D. Shaffer, R. J., and Thorson, L. D. 1982. "Some Effects of Stress, Friction, and Fluid Flow on Hydraulic Fracturing." SPE Paper 9831PA: $321-332$. 
Hubbert, M. K. and Willis, D. G. 1957. "Mechanics of Hydraulic Fracturing." Society of Petroleum Engineers, SPE Paper 686-G.

Janssen, M., Zuidema, R.J.H. and Wanhill, J. Fracture Mechanics. New York. Spon Press Taylor \& Francis, 2004.

Karihaloo, B.L. and Xiao, Q. Z. 2003. "Modelling of Stationary and Growing Cracks in FE Framework without Remeshing: A State-of-the-Art Review. Computers and Structures, 81 (2003) 119-129.

Kim, C. M., and Abass, H. H. 1991. "Hydraulic Fracture Initiation from Horizontal Wellbores : Laboratory Experiments." Presented at the 1991 US Symposium on Rock Mechanics, U. of Oklahoma, Norman,OK, July 10-12, 231-240.

Laborde, P., Pommier, J., Renard, Y. and Salaün, M.. 2005. "High-Order Finite Element Method for Cracked Domains" International Journal for Numerical Methods in Engineering 64 (3): 354-381.

Lecampion, B. 2009. “An Extended Finite Element Method for Hydraulic Fracture Problems" Communication in Numerical Methods in Engineering 25 (2): 121-133.

Locke, S. 1981. "An Advanced Method for Predicting the Productivity Ratio of a Perforated Well.” Journal of Petroleum Technology, 33 (12): 2481-2488.

Melenek, J.M., and Babuska, I. 1996. “The Partition of Unity Finite Element Method: Basic Theory and Applications" Computer Methods in Applied Mechanics and Engineering 139 (1-4): 289-314.

Moës, N., Dolbow, J. and Belytschko, T. 1999. “A Finite Element Method for Crack Growth without Remeshing" International Journal for Numerical Methods in Engineering 46 (1): 131-150.

Oil and Shale Gas Discovery News. "Monterey Shale.” Last Modified February 2014. http://oilshalegas.com/montereyshale.html.

PetroWiki. "Perforating Design.” Last Modified 26 March 2014. http://petrowiki.org/Perforating_design. 
Reeder, J. R. and Crews, J.H. 1990. "Mixed-Mode Bending Method for Delamination Testing." AIAA Journal 28 (7): 1270-1276.

Romero, J., Mack, M. G. and Elbel, J. L. 1995. “Theoretical Model and Numerical Investigation of Near-Wellbore Effects in Hydraulic Fracturing" SPE Paper 30506: 569578.

Serra, O. 1989. "Formation Microscanner Image Interpretation." Houston, Texas: Schlumberger Educational Services.

Soliman, M. Y. 1990. "Interpretation of Pressure Behavior of Fractured, Deviated, and Horizontal Wells": SPE Paper 21062.

Song, J.H., Areias, P. and Belytsckho, T. 2006. "A Method for Dynamic Crack and Shear Band Propagation with Phantom Nodes" International Journal for Numerical Methods in Engineering 67: 868-893.

Strouboulis, T. and Copps, K. 2001. "The Generalized Finite Element Method" Computational Methods for Applied Mechanical Engineering 190 (32-33): 4081-4193.

Venkitaraman, A., Behrmann, L. A. and Chow, C. V. 2000. "Perforating Requirements for Sand Control.” paper SPE 58788, presented at the SPE International Symposium on Formation Damage Control, Lafayette, Louisiana, USA, February 23-24.

Ventura, G. 2006. "On Elimination of Quadrature Subcells for Discontinuous Functions in the Extended Finite Element Method" International Journal for Numerical Methods in Engineering 66: 761-795.

Weber, N., Siebert, P., Willbrand, K., Feinendegen, M., Clauser, C. and Fries, T. P. 2013. "The XFEM With An Explicit-Implicit Crack Description For Hydraulic Fracture Problems", RWTH Aachen University.

Westergaard, H. M. 1940. "Plastic State of Stress Around a Deep Well” Journal of Boston Society of Civil Engineering 27: 387-391.

Wikipedia. "Partition of Unity." Last Modified 26 April 2013.www.wikipedia.org. 
Xia, L., Zhen-Zhong D. and Wohlever, C. 2012. "Hydraulic Fracture Simulation with an Extended Finite Element Method" Patent EP2672409 A2

Xiao, Q. Z., and Karihaloo, B. L. 2006. "Improving the Accuracy of XFEM Crack Tip Fields Using Higher Order Quadrature and Statically Admissible Stress Recovery" International Journal for Numerical Methods in Engineering 66 (9): 1378-1410.

Zienkiewics, O., Taylor, R. and Zhu, J. Z. 2000. The Finite Element Method: Its Basis and Fundamentals. 7th Edition. Burlington, MA: Elsevier, 